\title{
UCRL-TR-224095-REV-1
}

LAW RENCE LIVERMORE N A T IO N A L LABORATORY

\section{Final Report on X-ray Yields from OMEGA II Targets}

K. B. Fournier, M. J. May, S. A. MacLaren, C. A. Coverdale, J. F. Davis

June 21, 2007 
This document was prepared as an account of work sponsored by an agency of the United States government. Neither the United States government nor Lawrence Livermore National Security, LLC, nor any of their employees makes any warranty, expressed or implied, or assumes any legal liability or responsibility for the accuracy, completeness, or usefulness of any information, apparatus, product, or process disclosed, or represents that its use would not infringe privately owned rights. Reference herein to any specific commercial product, process, or service by trade name, trademark, manufacturer, or otherwise does not necessarily constitute or imply its endorsement, recommendation, or favoring by the United States government or Lawrence Livermore National Security, LLC. The views and opinions of authors expressed herein do not necessarily state or reflect those of the United States government or Lawrence Livermore National Security, LLC, and shall not be used for advertising or product endorsement purposes.

This work performed under the auspices of the U.S. Department of Energy by Lawrence Livermore National Laboratory under Contract DE-AC52-07NA27344. 


\title{
Final Report on X-ray Yields from OMEGA II Targets
}

28 May 2007

\author{
Kevin B. Fournier, Mark J. May, Stephen A. MacLaren \\ Lawrence Livermore National Laboratory
}

Christine A. Coverdale

Sandia National Laboratory

John F. Davis

Alme \& Associates

\begin{abstract}
We present details about X-ray yields measured with Lawrence Livermore National Laboratory (LLNL) and Sandia National Laboratories (SNL) diagnostics in soft and moderately hard X-ray bands from laser-driven, doped-aerogel targets shot on $07 / 14 / 06$ during the OMEGA II test series. Yields accurate to $\pm 25 \%$ in the $5-15 \mathrm{keV}$ band are measured with Livermore's HENWAY spectrometer. Yields in the sub-keV to $3.2 \mathrm{keV}$ band are measured with LLNL's DANTE diagnostic, the DANTE yields are accurate to $10-15 \%$. SNL ran a PCD-based diagnostic that also measured X-ray yields in the spectral region above $4 \mathrm{keV}$, and also down to the sub-keV range. The PCD and HENWAY and DANTE numbers are compared. The time histories of the moderately hard (hv $>4 \mathrm{keV}) \mathrm{X}$-ray signals are measured with LLNL's H11 PCD, and from two SNL PCDs with comparable filtration. There is general agreement between the H11 PCD and SNL PCD measured FWHM except for two of the shorter-laser-pulse shots, which is shown not to be due to analysis techniques. The recommended X-ray waveform is that from the SNL PCD p66k10, which was recorded on a fast, highbandwidth TDS 6804 oscilloscope. X-ray waveforms from target emission in two softer spectral bands are also shown; the X-ray emissions have increasing duration as the spectral content gets softer.
\end{abstract}

\section{Introduction}

This report represents a summary of the analysis of the data from various spectral diagnostics run by LLNL on the 14 July 2006 joint LLNL-SNL-AWE-MDA-DTRA SGEMP and coupon-exposure tests at the OMEGA laser [1] (Laboratory for Laser Energetics, University of Rochester), aka OMEGA II. Complimentary data to the LLNL diagnostics are given by Christine A. Coverdale of Sandia National Laboratory (SNL), which we also report. These are final, recommended assessments of the X-ray output from the Ge-loaded aerogel targets, estimated after several iterations between all parties following the tests. The X-ray source (aerogel targets) for each test was irradiated with either 1 or 3-ns-duration laser pulses. Time-integrated spectral coverage was obtained with a four-channel crystal spectrometer (HENWAY) [2] that simultaneously recorded emission in the $1-2,1.5-4$, and $5-15 \mathrm{keV}$ spectral bands. Initial inspection indicates that the A channel (the $5-15 \mathrm{keV}$ band) gave reasonable data, while the $\mathrm{B}$ and $\mathrm{D}$ 
channels $(1.5-4$, and $1-2 \mathrm{keV}$, respectively) seem to have had problems that prevented any useful data from being recorded. (The B channel, with a different filter configuration, was run on the OMEGA I test series $(03 / 11 / 2005)$ and gave very nice data in the $1.6-4 \mathrm{keV}$ spectral range $[3,4]$. A compendium of film images from three similar shot series is shown in Appendix C.) The high-energy signals from HENWAY channel A have absorption edges in them due to the presence of Fe and Ti foils in the employed filter pack. In order to remove noise just above the iron K-edge, fits to the continuum in the HENWAY data in the energy region between 8 and $15 \mathrm{keV}$ have been used to smooth the continuum signal in each spectrum (there were three shots in the OMEGA I test series that did not have any high-Z foils in the HENWAY A filter pack, the continua from those shots have been used to confirm the functional form used for fitting the continuum on each shot for OMEGA II). The X-ray sources for OMEGA II appear, according to the HENWAY data, to yield between 140 and $330 \mathrm{~J}$ into $4 \pi$ for all photon energies greater than $4 \mathrm{keV}$.

SNL provided time-resolved data from an array of nine photo-conductive devices (PCDs, only eight were operational), which had different filtration on each PCD, letting one reconstruct the emission by differencing the signals in the spectral window of each filtered PCD [5]. LLNL also provided a 13 channel X-ray diode (XRD) array called DANTE [6], also with different filtration on each channel used. The first 11 DANTE channels are designed to provide finely resolved coverage below $3 \mathrm{keV}$; the time resolved signals are integrated to give the total X-ray energy through the filters; the differential signals (after correcting for filter transmission and diode response) are then used to reconstruct the source spectrum. The DANTE signals from $1 \mathrm{eV}$ to approximately 3.5 $\mathrm{keV}$ provide the low-energy spectral data that compliment the HENWAY channel A spectra (and replace the apparently useless HENWAY channel B data). Two additional DANTE channels were added to measure the signal above $9 \mathrm{keV}$ and above $15 \mathrm{keV}$; these two channels have not yet been analyzed, although the signals in these channels do not show responses that are clearly above the noise levels, indicating that no useful information will be obtained. The DANTE spectrum for each shot below $3.5 \mathrm{keV}$ has been smoothly joined to the extrapolated fit to the measured HENWAY continuum from the higher energy HENWAY channel A data; the combined spectral regions have been distributed as the recommended spectra to be used in analysis on 08/27/2006. Further details on the diagnostics and analysis of hard- and soft-spectral data are given in Section II. Confirmation of our DANTE data reduction technique is obtained by comparing the DANTE results for a series of shots on 24 February 2006 to equivalent data from an independently built and analyzed diagnostic provided by the French Commissariat à l'Energie Atomique (CEA) called DMX; details of the DMX data reduction methods are given in Appendix D.

LLNL also ran a PCD in port H11 that had filtration of 10 mils of Be and $1 / 2$ mil of $\mathrm{Al}$ in front of it, thus transmitting only X-rays with energies above $4 \mathrm{keV}$. The temporal signal of the H11 PCD (after compensating the measured signal for high-frequency cable losses) is analyzed to give the temporal shape of the (harder-energy) X-ray waveform. This signal is also compared to the same signal from two of the more heavily filtered channels on the SNL PCD array. The two "3 ns shots" (44152 and 44157) show broader X-ray histories than the others shots, with X-ray signals that last $3 \mathrm{~ns}$ in total, but which have full widths at half maxima (FWHM) that are closer to $1.6 \mathrm{~ns}$. There are two shots in 
the " 1 ns" set of shots (44153 and 44158) that show interesting late time features that indicate hydrodynamic motion of the Be target holders may have a strong effect on the final X-ray fluence from the doped-aerogel target. Details of the PCD unfold and analysis are given in Section III. Waveforms of the X-ray signals in softer bands, from more lightly filtered SNL PCDs and soft-filtered DANTE channels are also presented. It is seen that the X-ray waveform from the target is strongly temporally broadened as the transmitted X-ray energy gets softer.

Comments on the evolution of the data from the film images to the final, distributed, smoothed data are given in Appendix A, along with technical details about the hardware and software that makes up the DANTE and LLNL PCD diagnostics (mostly repeated from Section II). Appendix A also contains comments about variation in results for the temporal waveforms of the X-ray pulses that results from different methods of analysis. Since the signal in the SGEMP probes effectively is the time derivative of the X-ray waveform, one should use the actual measured X-ray signal in any analysis, rather than, say, a Gaussian approximation.

Plots that show the variation in measured X-ray yields for nominally identical shots as well as plots that summarize the variation in the X-ray source as a function of different parameters (density, size, intensity) are given in Appendix B. This is offered in order to give one an idea of the variability users of the X-rays from doped aerogels can expect at the OMEGA laser. The interesting physics that underlies the target performance (and variation) is left to a pair of papers being written for the journals High Energy Density Physics and Physics of Plasmas.

\section{Data ( $2^{\text {nd }}$ cut - resulting in distribution of recommended spectra)}

\section{II.a Hard X-ray Data}

Table I shows the HENWAY yields estimated from the Q-PET-crystal channel (channel A). The individual spectra were assessed from a region of the recording medium (DEF film) with filtration of $1 \mathrm{mil} \mathrm{Be}+18 \mu \mathrm{m} \mathrm{Al}+6 \mu \mathrm{m} \mathrm{Ti}+12.7 \mu \mathrm{m} \mathrm{Fe}$ (socalled cut 'c'). Digitized images of some of the HENWAY films from OMEGA II are shown for all HENWAY channels in Appendix C. The reported yields in Table I are for the $\mathrm{Ge}^{30+} \mathrm{K}$-shell line complex $(9-15 \mathrm{keV})$ and for the whole recorded spectrum (approximately $4.0-15 \mathrm{keV}$ ). Cuts through regions of the film for each shot with different filtration (e.g., with 12 instead of $18 \mu \mathrm{m}$ of Al, so-called cut 'd', or with $25 \mu \mathrm{m}$ of $\mathrm{V}$ added, namely, cut 'a') give yields that agree to within $10 \%$ with the recommended spectral data (cut 'c'). This means that the relative thicknesses of the filter materials are well known for the configuration as built. A background 'fog' level is assessed for each shot by taking a cut horizontally across the film image from an unexposed region of the film (usually below cut 'a'). For HENWAY channel A, the result is data with K-shell Ge lines, K-shell absorption edges, and a smooth continuum (reduced spectra from OMEGA I, 02/24/06 and OMEGA II shots are compared in Appendix A). The observed continuum emission in each HENWAY spectrum in the region from 8 to $13 \mathrm{keV}$, but away from the Ge K-shell lines, has been fit to an exponential form. This form was then used to extrapolate a smooth signal for the HENWAY data below the Fe K-edge at 7.11 
$\mathrm{keV}$. The product of the $\mathrm{Ti} \mathrm{K}$-edge and the Fe K-edge made the signals around and below $7 \mathrm{keV}$ particularly susceptible to noise. The ability of the single-exponential form for fitting the data down to approximately $4 \mathrm{keV}$ was checked against the three shots from the OMEGA I test that did not have Fe foils in the filter pack: the fits are excellent.

Also listed for comparison is the derived signal from two of SNL's higher-energy PCD channels (all emission from approximately $4 \mathrm{keV}$ up). The PCDs were P66k10 and P126kt, which were standard $0.5 \mathrm{~mm}$ thick diamond PCDs, filtered with 10 mil Kapton and $15.6 \mu \mathrm{m} \mathrm{Al}$. The PCDs were operated at a bias level that was on the order of $100 \mathrm{~V}$. The details of the PCD operation and data unfolding are being written up by Christine Coverdale for a Sandia report and will be available there [5]. The PCD-derived yields range from $15 \%$ smaller to $130 \%$ larger than those of HENWAY, and are generally $20-$ $50 \%$ larger. The two three-ns pulses had observably lower yields than most of the one-ns pulses (see also Fig. B.4).

\begin{tabular}{|c|c|c|c|c|c|c|}
\hline Shot no. & $\begin{array}{c}\text { Pulse } \\
\text { width } \\
\text { (ns) }\end{array}$ & $\begin{array}{c}\text { HENWAY } \\
(\mathrm{J} / \text { sphere })\end{array}$ & $\begin{array}{c}\text { HENWAY } \\
(\mathrm{J} / \text { sphere })\end{array}$ & $\begin{array}{c}\text { PCD } \\
(\mathrm{J} / \text { sphere })\end{array}$ & $\begin{array}{c}\text { Difference } \\
(\%)\end{array}$ & $\begin{array}{c}\text { Te } \\
(\mathrm{keV})\end{array}$ \\
\hline & & & & & & \\
\hline 44152 & 3 & 42.5 & 190.8 & 161.4 & -15.4 & 1.989 \\
\hline 44153 & 1 & 54.3 & 139.7 & 317.0 & 126.9 & 2.160 \\
\hline 44154 & 1 & 73.7 & 220.3 & 337.9 & 53.4 & 2.308 \\
\hline 44156 & 1 & 90.5 & 266.0 & 374.4 & 40.8 & 2.518 \\
\hline 44157 & 3 & 70.5 & 205.4 & 203.8 & -0.8 & 2.210 \\
\hline 44158 & 1 & 142.3 & 331.1 & 391.9 & 18.4 & 3.111 \\
\hline
\end{tabular}

Table I - Hard X-ray yields (isotropically into all solid angles) from the HENWAY spectrometer (channel A: Q-PET crystal, filtered with Be, Al, Fe and Ti) and SNL's PCDs (P66k10, P126kt, filtered with 10 mil Kapton and $15.6 \mu \mathrm{m} \mathrm{Al})-P C D$ data updated 4/07. The first HENWAYyield number is integrated in the band $9-15 \mathrm{keV}$, the second HENWAY-yield number is integrated over the whole range shown in Figs. 1 - 3 (approximately $4.5-15 \mathrm{keV}$ ), plus extrapolation down to $4.0 \mathrm{keV}$, which is approximately the same band recorded by the SNL PCDs. The difference relative to the HENWAY numbers is given in the $6^{\text {th }}$ column. The plasma temperature found from fitting the continuum in each HENWAY spectrum is given in the last column.

All shots (based on HENWAY-derived yields) performed below the pre-shot predictions, which were based on 02/24/06 shots 42750 and 42751 (176.6 and 77.0 $\mathrm{J} /$ sphere in the $9-15 \mathrm{keV}$ band for 1 and $3 \mathrm{~ns}$ shots, respectively). NOTE: PET crystal reflectivity data is based on fits to reflectivity curves in the literature (Henke [7] and Gilfrich [8]), and apocryphal knowledge of measurements done at LLNL years ago (in the energy region around $4.7 \mathrm{keV}$, and maybe other regions). Also, the filter transmission is based on foil thickness quoted by the vendor along with quoted uncertainties (new HENWAY filters for each channel were built for OMEGA II, foils were purchased from Goodfellow, www.goodfellow.com); the actual transmission of the assembled filter packs has not been measured with a calibrated X-ray source. These two areas of uncertainty are being addressed in the near future with actual bench-top calibration runs. The HENWAY-derived yields in Table I are uncertain to $\pm 25 \%$. The final HENWAY 
spectra for the six OMEGA II shots are shown in Figs. 1 - 3 (intermediate, reduced data, before the continuum fitting and smoothing, are given in Appendix A). A comparison of two of our (best performing) measured spectra with the pre-shot 'reference spectra' from 42750 and 42751 is given in Fig. 4.

The results for shot 44158 (the highest yield shot in OMEGA II) are fluences of $92 \mathrm{~mJ} / \mathrm{cm}^{2}$ at a standoff distance of $18 \mathrm{~cm}$ (the location of LOS 3 and 1, TIMs 2 and 3) and $74 \mathrm{~mJ} / \mathrm{cm}^{2}$ at $20 \mathrm{~cm}$ (the location of LOS 2 and 4, TIMs 4 and 6), while for the lowest-yield 1-ns shot (44153), the results are 50 and $40 \mathrm{~mJ} / \mathrm{cm}^{2}$, respectively. For the highest-yielding $3 \mathrm{~ns}$ pulse, 44157, the fluence numbers are also 50 and $40 \mathrm{~mJ} / \mathrm{cm}^{2}$ at 18 and $20 \mathrm{~cm}$, respectively. These fluence numbers are found by averaging the HENWAY and SNL PCD yields in the spectral band above $\approx 4.0 \mathrm{keV}$. Based on the spectrum for 44156 , these spectra had an (intensity-weighted) average energy of $7.6 \mathrm{keV}$.

\section{II.b Soft X-ray Data}

Table II has the time- and energy-integrated output from the Ge-doped targets between 1 and $3.2 \mathrm{keV}$ as measured by DANTE [6]. The DANTE diagnostic had 11 differently filtered X-ray diode (XRD) channels in the band that ranged from sub-keV to $3 \mathrm{keV}$, thus providing a well resolved spectral signal. The model number for the diodes in DANTE is the XRD-31, a fast, windowless X-ray vacuum photodiode developed by EGG. It is currently the primary fast X-ray detector used to diagnose the X-rays on NIF and OMEGA on the multichannel DANTE spectrometer. The XRD-31 has a dynamic range of less than 1e-12 amps to about 10 amps. A measured response time for five XRD-31s assembled in early 2004 was found to be $149.7 \pm 2.75$ ps by Don Pellinen (NSTec - Formerly Bechtel). The signals are recorded onto SCD5000 scopes (approximately $200 \mathrm{Gs} / \mathrm{s}$ ) with $\approx 50 \mathrm{GHz}$ sampling (19.55 ps per point). The DANTE analysis software has been run with a cable-compensation correction (however, it is unclear of the origin of the data used, or if software compensation is the best solution).

\begin{tabular}{|c|c|c|c|c|}
\hline Shot no. & $\begin{array}{c}\text { Pulse width } \\
(\mathrm{ns})\end{array}$ & $\begin{array}{c}\text { DANTE } \\
(\mathrm{J} / \mathrm{ster})\end{array}$ & $\begin{array}{c}\text { SNL PCD } \\
(\mathrm{J} / \mathrm{ster})\end{array}$ & $\begin{array}{c}\text { Difference } \\
(\%)\end{array}$ \\
\hline & & & & \\
\hline 44152 & 3 & 333.1 & 294.4 & -11.6 \\
\hline 44153 & 1 & 380.2 & 311.9 & -18.0 \\
\hline 44154 & 1 & 512.1 & 290.9 & -43.2 \\
\hline 44156 & 1 & 508.4 & 310.8 & -38.9 \\
\hline 44157 & 3 & 225.5 & 262.6 & 16.5 \\
\hline 44158 & 1 & 369.6 & 322.1 & -12.9 \\
\hline
\end{tabular}

Table II - Soft X-ray yields (J/ster) measured with DANTE, which was oriented $37.3^{\circ}$ with respect to the target axis, and with a SNL PCD. DANTE yields are integrated from measured spectral yield between 1.0 and $3.2 \mathrm{keV}$. The DANTE spectra for this set of six shots are shown in Figs. 5 and 6 . Note, there is a large amount of energy below $1 \mathrm{keV}$; the spectral region above 3.5 $\mathrm{keV}$ is (as of yet) undependable since the response functions for the two DANTE channels that cover this region are being developed. Also shown is the soft X-ray yield for each shot from the SNL PCD filtered with unknown (possibly p122, $8 \mu \mathrm{m}$ of kapton, or p123, 1 mil of kapton), 
giving an estimate of emission with $\mathrm{h} v>0.3-1 \mathrm{keV}$, as well as the percentage difference between the two measurements.

The DANTE viewed the face of our target at an angle of $37.3^{\circ}$ with respect to the target's axis thus having a good, unobstructed view of the target's face. In the unfolding of the DANTE signals, assumptions are made about the view that the diagnostic has into the interior of the emitting chamber. In many (most) LLNL high-energy-density experiments, the DANTE is looking into the laser entrance hole (LEH) of a hohlraum with gold or high-Z-cocktail walls (thicknesses vary, but usually $\approx 25 \mu \mathrm{m}$ ). Consequently, the DANTE unfold software corrects for the unseen volume in the emitting chamber with the assumption that no flux is seen through the chamber walls. In our case, the walls of our 'hohlraum' were made of $75( \pm 5) \mu \mathrm{m} \mathrm{Be}$; there is a fair chance that in some of the DANTE channels with response near $3 \mathrm{keV}$, some emission from the whole volume of the aerogel-filled cylinder entered the detector. Thus, our estimates, which assume no emission from anywhere other than the LEH, may be systematically high-sided. The DANTE yields in the $1-3.5 \mathrm{keV}$ may be $10-15 \%$ too high. A comparison of yields estimated from a shot series on 24 February, 2006 with DANTE and an independently calibrated and analyzed diagnostic is given in Appendix D; the result is high confidence in the DANTE measurements for these aerogel targets.

Also listed in Table II are the values from the "soft" channel(s) of the SNL PCDs (converted from all solid angle to per steradian). The specific identifiers for the PCD(s) (probably the SNL PCD labeled p123, or that one averaged with another) and the scope(s) used to record these data (either TDS $6804 \mathrm{~b}$ or TDS 694c models) are unknown. The agreement between the DANTE and PCD data in the stated band is quite good given all the caveats above, except for shots 44154 and 44156 . The signals from two additional HENWAY channels (Channel B $1.5-4 \mathrm{keV}$ and Channel D 1.5-2.1 keV) are still to be analyzed, if they can be analyzed at all; together, they should compliment the DANTE/PCD measurements. These two shots had the narrowest high-energy X-ray waveforms temporally speaking (see Fig. 11), and rather temporally extended soft X-ray signals (see Fig. 13).

The DANTE spectra for all shots (as connected to the HENWAY A signals) are shown in Figures 5 and 6. NOTE: signals for the DANTE channels above $3.2 \mathrm{keV}$ are still unknown; we are still waiting on the response function for the two high energy channels that were run in order to complete the unfold of the flux above $3 \mathrm{keV}$, however, examination of the raw signal in these channels indicates little response above the noise in each channel. Pre-shot predictions for this spectral region were made based on spectra measured from 02/24/06 shots with the Commissariat à l'Energie Atomique (CEA) DMX diagnostic [9]. The DMX is similar to DANTE, with a number of X-ray (Al, Ni, GaAs) diode channels that have different filtration; the DMX and DANTE have the same lineof-sight view of targets on the P5-P8 axis. A comparison of the DANTE spectra for two of our shots with the DMX-measured 'reference spectra' from 02/24/06 is shown in Figure 7; a comparison of the DANTE and DMX spectra for shots 42750 and 42751 looks very similar to the comparison in Fig. 7. For shot 42750, the DANTE and DMX yields in the 30 to $3000 \mathrm{eV}$ band are 810.8 and $725.8 \mathrm{~J} / \mathrm{sr}$, respectively, and for shot 42751 , in the same band, 602.5 and $676.5 \mathrm{~J} / \mathrm{sr}$, respectively. These numbers are the result of iterative analysis between LLNL and CEA researchers, and are current as of October 2006 (see Appendix D). Using the DANTE-measured yields in the $1-3.2 \mathrm{keV}$ band for 
44152 and 44154, we find a fluence of 0.37 and $0.57 \mathrm{~J} / \mathrm{cm}^{2}$ at a distance of $30 \mathrm{~cm}$, approximately the standoff distance the coated optical samples had.

A summary plot of the recommended spectra is shown in Fig. 8. From the figure, and Tables I and II, it is seen that the highest-yielding hard-X-ray shot (44158, highest by a lot) is the fourth highest-yielding shot in the soft band. The two shots that had the fastest X-ray waveforms $(44154,44156)$, based on hard-X-ray signals, also have the largest soft-X-ray yield (by DANTE, and the SNL PCDs), while shot 44153, which has a very odd temporal profile (see Fig. 12 below), was weakly performing in the hard-X-ray band, but quite strongly performing in the soft-X-ray band.

\section{Time response (to be correlated with SGEMP test-object response)}

\section{III.a Hard X-ray Waveforms}

Table III lists the full-width at half maxima (FWHM) for the delivered laser power to the targets in our 3- and 1-ns configurations. The 3-ns configuration had an envelope that lasted for 3-ns with a fairly Gaussian power profile. The FWHM for the laser power for those shots were 1.6-1.7 ns, however, we have continued to refer to those shots as ' 3 -ns shots' in this report. Examples of the laser power delivered to the P8 face of the target for both pulse shapes are shown in Figure 9. It is seen that the laser power to the target is very reproducible.

Table III also listed the measured FWHM for the X-ray signal from the aerogel targets as measured by LLNL's PCD in port H11 and the average of the SNL p66k10 and p126k10 PCDs, both of which were filtered to be sensitive to X-rays with energies above $3.5-4.0 \mathrm{keV}$. The "standard" PCDs used by LLNL are $1 \times 1 \times 1 \mathrm{~mm}$ cubes. (We have one PCD that is $2 \mathrm{~mm}$ long, but it is not used in the H-11 location.) The signals are recorded onto the SCD5000 scopes used for DANTE. There was a second LLNL PCD in port P11 that has been lost due to construction for the OMEGA EP project. The H11 PCD was filtered with 10 mil Be and $1 / 2$ mil Al so that it saw X-ray emission above 3.5 $\mathrm{keV}$. The PCD-data-reduction routine uses an analytic response function that is derived to match the response of the PCD/bias-T/cable combination to a short pulse as measured in the lab to cable compensate the recorded PCD signal. After unfolding the time response, the analysis corrects for the attenuators and for the $800 \mathrm{~V}$ bias; the bias correction is applied after the attenuator correction. Thus, the data is reduced to the voltage as seen at the PCD. Further conversion to flux would require a separate crosscomparison (typically with Dante) to evaluate the PCD's sensitivity. The H11 PCD FWHM have been obtained by fitting a Gaussian envelope to the observed, reduced Xray waveforms using the curve-fitting capabilities of the KaleidaGraph ${ }^{\mathrm{TM}}$ program.

The SNL PCD FWHM have been obtained from the cable-compensated, biascorrected signals from PCDs p126k10 and p66k10 by Christine Coverdale. The p126k10 signals were recorded on a TDS $694 \mathrm{C}$ oscilloscope, which had $10 \mathrm{Gs} / \mathrm{s}$ sampling, and 3 $\mathrm{GHz}$ analog bandwidth, the p66k10 signals were recorded on a TDS 6804B oscilloscope, which had $20 \mathrm{Gs} / \mathrm{s}$ and $8 \mathrm{GHz}$ bandwidth. The p66k10 signal is more appropriate for analyzing truly high-frequency phenomena. Both waveforms should be within the ability of the oscilloscopes to record accurately. Both PCDs were filtered with 10 mils of 
Kapton and $15 \mu \mathrm{m}$ of $\mathrm{Al}$ (thus, they were sensitive to emission generally above $4 \mathrm{keV}$ ). The SNL FWHM were found by using the XDAMP data-analysis program to take, literally, the full width at half the maximum signal for each waveform using cross hairs. The average of the reported FWHM from the two SNL PCDs for all six shots in the OMEGA II series has been listed in Table III. The difference between the Gaussian fit, and the XDAMP analysis, is that the XDAMP analysis can ignore broad shoulders and large asymmetries that will affect the quality of a Gaussian fit. However, for most of the waveforms, the Gaussian approximation seems quite good for the data recorded on LLNL's SCD5000 scopes. The results for shots 44153 and 44158, both of which had unusual, and non-Gaussian shapes, differ markedly between the LLNL and SNL analysis. Also, in the case of the two 3-ns shots (44152 and 44157), the LLNL-derived H11 FWHM is substantially longer than that of the SNL-derived SNL PCD FWHM.

Gaussian fits to the SNL waveforms, using the same technique as was used for the H11 data, are given in Appendix A (Table A.III) for both the p66k10 and p126k10 PCDs. There, the LLNL fits to the SNL data are quite close to the SNL-derived FWHM, indicating that the differences in Table III are due to the different responses of the LLNL and SNL PCDs, and not to the method of analysis used. It is recommended that for applications that need the temporal waveform of the hard X-ray signal, one should use the measured waveforms from SNL PCD p66k10 (see Figs. 10-12).

\begin{tabular}{|c|c|c|c|}
\hline Shot no. & $\begin{array}{c}\text { Laser FWHM } \\
(\mathrm{ns})\end{array}$ & $\begin{array}{c}\text { H11 PCD FWHM } \\
(\mathrm{ns})\end{array}$ & $\begin{array}{c}\text { SNL PCD FWHM } \\
(\mathrm{ns})\end{array}$ \\
\hline 44152 & 1.68 & 1.97 & 1.48 \\
\hline 44153 & 1.00 & 1.54 & 1.80 \\
\hline 44154 & 1.00 & 1.02 & 1.13 \\
\hline 44156 & 1.00 & 1.02 & 1.18 \\
\hline 44157 & 1.71 & 1.64 & 1.53 \\
\hline 44158 & 1.00 & 1.74 & 1.28 \\
\hline
\end{tabular}

Table III - Measured laser power on target, H11-PCD-measured FWHM for the OMEGA II shots. The H11 PCD looked nearly face-on to the cylindrical aerogel target and was filtered with 10 mil Be and $1 / 2$ mil $\mathrm{Al}$ (so it saw emission essentially above $3.5 \mathrm{keV}$ ). Also listed are the measured FWHM for the hard X-ray output from the SNL PCDs, averaged from signals from two PCDs, which were recorded with a TDS694C and TDS6804B oscilloscope.

The X-ray waveforms for all shots as measured by the SNL PCDs and the H11 PCD are compared in Figures 10 - 12. In the analysis of the H11 PCD, the data are cable compensated for high-frequency signal losses, and the calibrated PCD voltage is obtained given the diode's response function and the filter transmission. The shape of the (recommended) SNL PCD waveforms and the LLNL H11 PCD waveforms are qualitatively very similar for each shot in the OMEGA II series, as can be seen in Figs. 10-12. In most cases, it appears the X-ray output follows the laser power quite closely, particularly in the two 1 ns cases of 44154 and 44156 (Fig. 11). For the 3 ns pulses (Fig. 10), the X-ray waveforms show peak X-ray signals after the peak of the laser power, and nearly simultaneous with the third plateau in the laser power (which corresponds to the firing of the low-angle cone 1 beams). The measured waveform for the second and sixth shots (both 1-ns-drive pulses), 44153 and 44158, are anomalous 
(Fig. 12); however, the extended emission time is consistent with responses seen on other diagnostics (SNL PCDs, DANTE channels, SGEMP test objects). The late-time peaking of the X-ray power in shot 44158 is due to hydrodynamic motion of the Be cylinder walls toward the cylinder axis, which results in a high-density emitting region. This phenomenon (and the resulting spike in the X-ray emission from the target) is seen in 2-D radiation-hydrodynamic simulations with the LASNEX code, and has been demonstrated in earlier OMEGA experiments and at the GEKKO XII laser. At present, we cannot explain why shot 44158 displayed such a strong hydrodynamic-compression effect while the other shots did not. The shape of the X-ray waveform for 44153, with an "early" peak in the X-ray power and a late-time shoulder is, at present, not understood. Analysis of the X-ray waveforms from the OMEGA I and the 02/24/2006 Ge-doped target shots is in progress, and, hopefully, will further elucidate the observed phenomena.

\section{III.b Soft X-ray Waveforms}

Figure 13 shows the hard X-ray signal from two SNL PCDs, p126 and p66 (10 mil kapton, $15 \mu \mathrm{m} \mathrm{Al}$ ), and the soft X-ray signal from one SNL PCD, p122 $(8 \mu \mathrm{m}$ kapton), for two of the one-ns-laser-irradiation shots $(44154,44156)$. In this case, the hard X-ray waveforms (also shown in Fig. 11) have a sharp, narrow pulse shape, with an approximate Gaussian waveform described in Table 3 (and in Table A.III). The two SNL PCDs filtered for the hard X-ray signals show very good consistency (as do PCD p126 and the LLNL H11 PCD in Fig. 11). The softer X-ray signal (green trace) in Fig. 13 has an earlier rise time than the harder X-ray signals, a width of an early peak that is comparable to the width of the hard X-ray pulses, and a rather broad late time pulse. The origin of this late-time pulse is most likely from recombination of the Ge and $\mathrm{Si}$ ions in the target plasma. All waveforms in Fig. 13 have been arbitrarily shifted to their displayed positions. Figure 14 compares the same measured X-ray waveforms for onens-laser-irradiation shots 44153 and 44158 (also shown in Fig. 12). For both these shots, the harder X-ray signals measured with SNL PCDs p66 and p126 have unexplained features that are not well approximated by the Gaussian fits in Table 3, and which are consistent between the two SNL PCDs. In both Figs. 13 and 14, the softer X-ray signals from SNL PCD p122 are much broader than the harder X-ray signals from the other two SNL PCDs.

Figure 15 compares the X-ray waveforms from four different SNL PCDs (all normalized) on shots 44152 and 44157 (both 3-ns-laser irradiation): p66k and p126k, p123 filtered with 1 mil kapton, and p122, all described above. The PCDs have an increasing contribution to the measured response from soft and very soft X-ray photons that results in the progressively broader X-ray waveforms. The hard X-ray signals have durations that are generally similar to the laser-power pulse shape (see Fig. 10), but which lag the rising edge of the laser power significantly. The PCD signals dominated by softer contributions (i.e., signals from p123 and p122) are much broader than the hard Xray signals and have qualitatively different shapes. Figures 16 and 17 compare the soft and softest X-ray signal from SNL PCD p122 (filtered with only $8 \mu \mathrm{m}$ of kapton) with signals from channels comparable channels from LLNL's DANTE diagnostic. The upper panels in Figs. 16 and 17 are compared with DANTE channels 6 and 7, both of which show the signal around the $1 \mathrm{keV}$ spectral band. The DANTE channel number 4, which 
is optimized for measuring emission between 450 and $520 \mathrm{eV}$, matches the PCD p122 signal very closely in the lower panel of Fig. 16. DANTE channel 4 failed to return data in shot 44157, but the two DANTE channels that bracket the emission window of channel 4 also bracket the SNL PCD. This analysis confirms that the waveforms measured by the SNL PCDs and LLNL PCDs and DANTE channels are robust. Thus, one can confidently tailor an experiment for both the temporal profile and spectral band of the driving X-rays from these laser-driven X-ray sources by using appropriate filtration.

\section{Summary and Conclusions}

Six Ge-doped aerogel targets were shot to create laser-driven plasma X-ray sources for the OMEGA II test series. Yields in the X-ray band from $4.5-15 \mathrm{keV}$ are found from HENWAY channel A (PET crystal) data that have been calibrated and a smooth continuum model has been fit to the data where there are no lines. There is decent agreement with the yields from higher-energy PCD channels fielded by SNL. Yields in the spectral band from 0.03 to $3.2 \mathrm{keV}$ have been derived from the differential signals in 11 channels of the diode-based DANTE instrument. Again, the DANTE yields and the SNL PCD yields show reasonable agreement, all of which may improve as the actual spectral distribution of the radiation is factored into the PCD analysis. The DANTE spectra and the HENWAY spectra have been smoothly joined to produce recommended spectra for the OMEGA II test series (Fig. 8), with recommended yields given in Tables I and II. Further spectral data may be derived from softer HENWAY channels (KAP and RAP crystals), but this seems unlikely as the film from those channels does not look anything like the (well understood) film from the OMEGA I test series. The DANTE spectra, in particular, are different from the pre-shot spectra used to make predictions, which came from the CEA's DMX instrument. Analysis following the OMEGA II test series of DANTE and DMX spectra measured from the same shots $(02 / 24 / 2006)$ has shown that DMX X-ray yields converge to the measured DANTE yields when the analysis accounts for thin spectral lines (see Appendix D). This, in part, explains the difference between measured spectra in the OMEGA II test series and the pre-shot predictions. Data from LLNL's H11 PCD have been compared to the measured laser power on the target and to the multi-keV X-ray waveforms from an eight-channel PCD array provided by SNL. Hydrodynamic phenomena that affect the X-ray yield (as well as the waveform) have been identified and modeling is underway. The agreement between the LLNL and SNL measurements for the multi-keV X-ray duration, rise time and fall time are all quite good. Analysis of the SNL PCDs that were filtered with much thinner filtration showed long duration X-ray waveforms for emission in the $1 \mathrm{keV}$ band and even longer emission in the sub-keV spectral range. The SNL waveforms are confirmed by comparison with measured X-ray fluxes from LLNL's DANTE diagnostic. The SNL-measured waveforms are recommended for any application that requires X-ray pulse-shape data.

This work was performed under the auspices of the U.S. Department of Energy by Lawrence Livermore National Laboratory under Contract No. W-7405ENG-48. Sandia is a multi-program laboratory operated by Sandia Corporation, a Lockheed Martin Company, for the U.S. Department of Energy under Contract DE-AC04-94AL8500. This work was also supported by the Defense Threat Redution Agency (DTRA) under the IACRO "Laser Plasma Radiation Source Development and Evalutaion". 


\section{Reference:}

[1] J. M. Soures, R. L. McCrory, C. P. Verdon et al., Direct-drive laser-fusion experiments with the OMEGA, 60-beam, $>40 \mathrm{~kJ}$, ultraviolet laser system, Phys. Plasma 3, 2108 (1996).

[2] L. N. Koppel and J. D. Eckels, High Resolution X-ray Crystal Spectrographs, Lawrence Livermore National Laboroatory Report UCRL-79781, October 1977.

[3] K. B. Fournier, D. E. Beutler, K. J. Dudley, et al., Conducting X-Ray Effects Experiments with Underdense X-Ray Sources on the OMEGA Laser Facility, Journal of Radiation Effects, Research and Engineering 20, pg. (2006). See UCRL-JRNL-213458.

[4] K. B. Fournier, et al., Absolute X-ray Yields from Laser-Irradiated, Ge-Doped Aerogel Targets, in Laser-Generated and Other Laboratory X-ray and EUV Sources, Optics and Applications, Proc. of SPIE vol. 5918, G.A. Kyrala, J-C Gauthier, C. MacDonald, A. Khounsary, editors, (SPIE, Bellingham, WA, 2005) $59180 \mathrm{~N}$.

[5] C. A. Coverdale, Sandia National Laboratory Report, in progress (2006).

[6] H. N. Kornblum, R. L. Kauffman, and J. A. Smith, Measurement of $0.1-3-\mathrm{keV}$ x rays from laser plasmas, Rev. Sci. Instrum. 57, 2179 (1986).

[7] B. L. Henke, E. M. Gullikson, and J. C. Davis, X-Ray Interactions: Photoabsorption, Scattering, Transmission, and Reflection at $\mathrm{E}=50-30000 \mathrm{eV}, \mathrm{Z}=1-92$, Atomic Data Nucl. Data Tables 54, 181 (1993).

[8] J. V. Gilfrich, D. B. Brown, and P. G. Burkhalter, Integral Reflection Coefficient of X-ray Spectrometer Crystals, Appl. Spectrosc. 29, 322 (1975).

[9] J. L. Bourgade, B. Villette, J. L. Bocher et al., DMX: An absolutely calibrated time-resolved broadband soft x-ray spectrometer designed for MJ class laser-produced plasmas (invited), Rev. Sci. Instrum. 72, $1173(2001)$.

\section{Distribution:}

TA Stringer - ITT

N Duncum - ITT

KJ Dudley - AWE

CA Coverdale - SNL

J Fisher - GHS

DE Beutler - SNL

D Osborne - AWE

JF Davis - A\&A

MT Tobin - MDA

D Newlander - GHS

MJ May - LLNL

C Sorce - LLE

SA MacLaren - LLNL

SB Hansen - LLNL

RC Webb - SAIC 
08/08/2006 - 1 ns comp plot

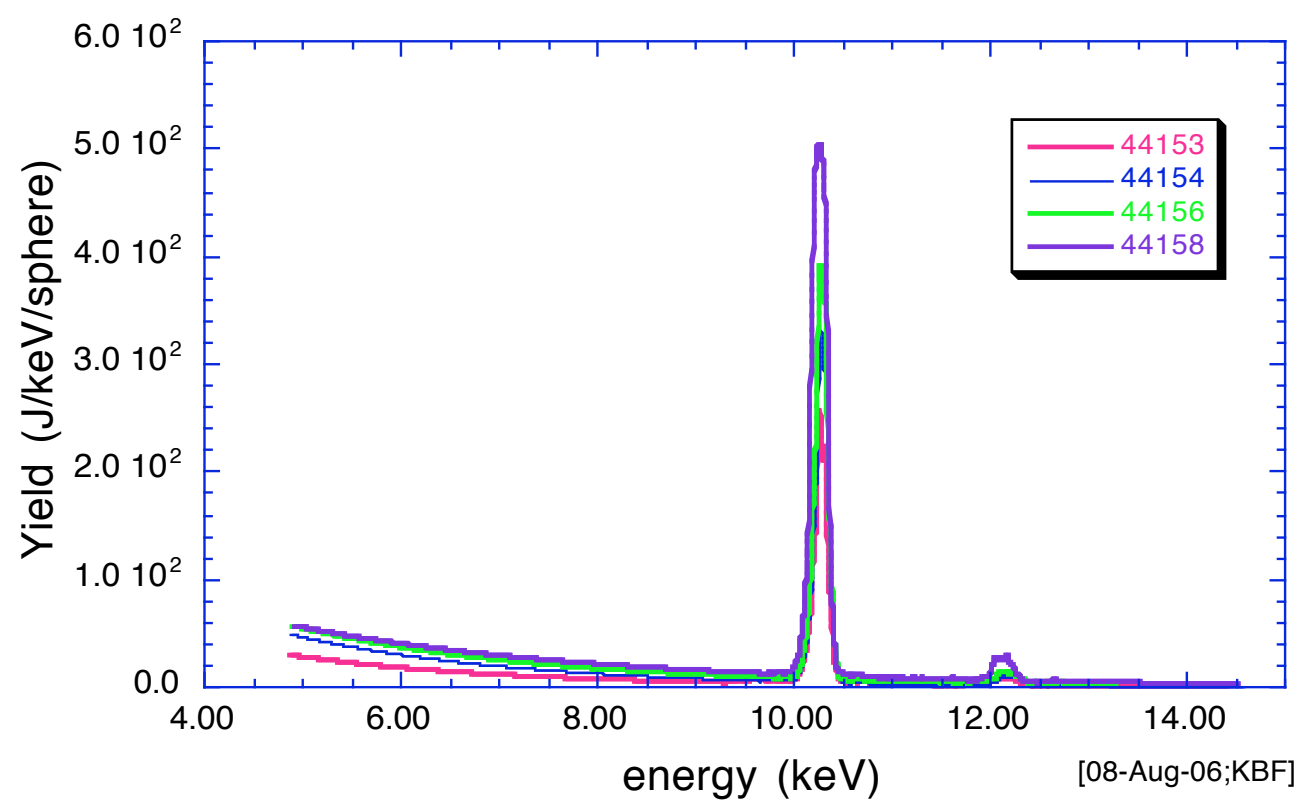

Figure 1 - HENWAY Channel A spectra for shots 44153, 44154, 44156, 44158 (1 ns, broad focusing, $3.7 \mathrm{mg} / \mathrm{cm}^{3}, 2.0 \mathrm{~mm} \times 2.2 \mathrm{~mm}$ [ID $\times$ length]).

08/08/2006 - 1 ns comp plot

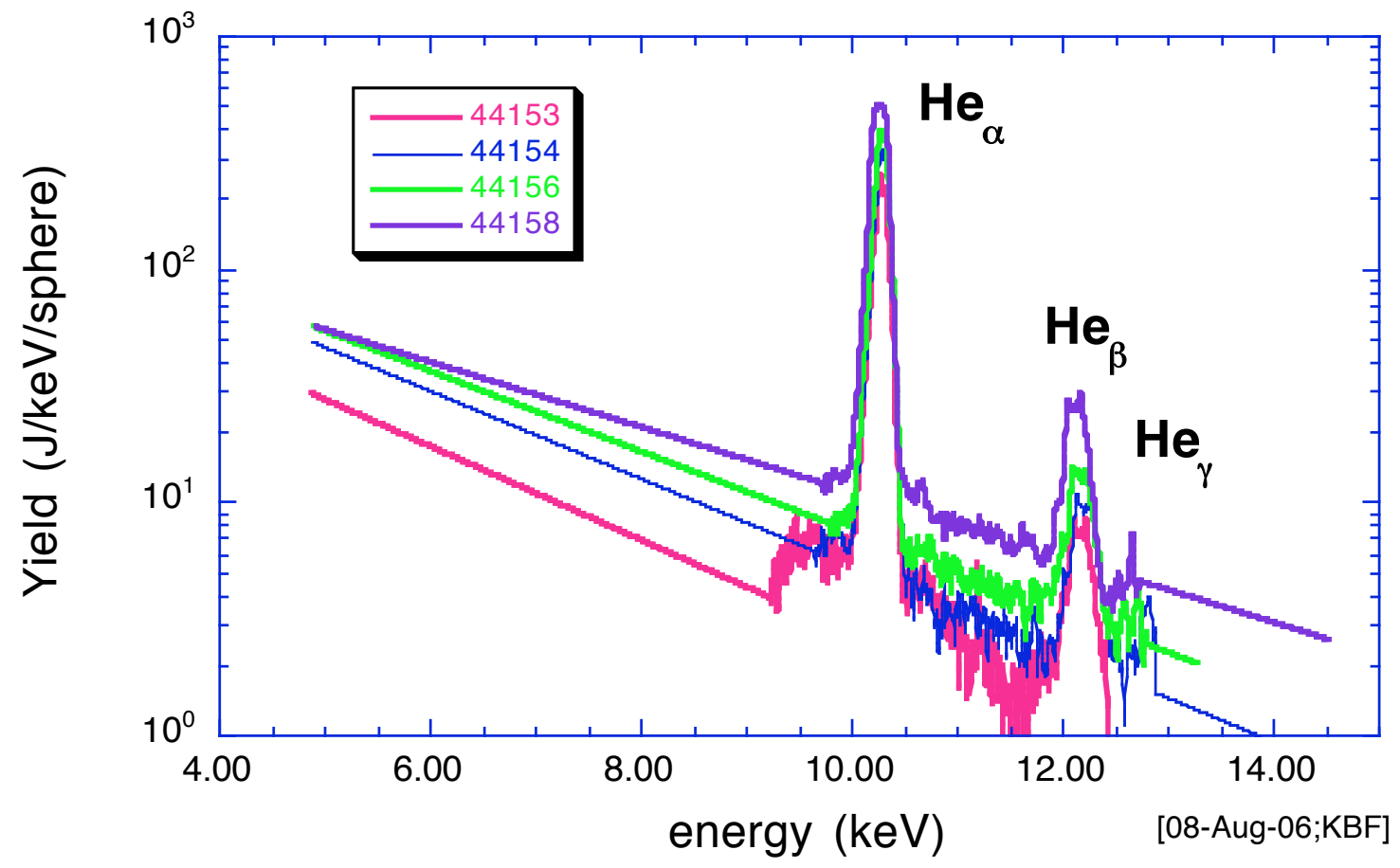

Figure 2 - HENWAY Channel A spectra for shots 44153, 44154, 44156, 44158 (1 ns, broad focusing, $3.7 \mathrm{mg} / \mathrm{cm}^{3}, 2.0 \mathrm{~mm} \times 2.2 \mathrm{~mm}$ [ID $\times$ length]) on a logarithmic scale. 
08/08/2006 Ge K-shell 3 ns comp plot

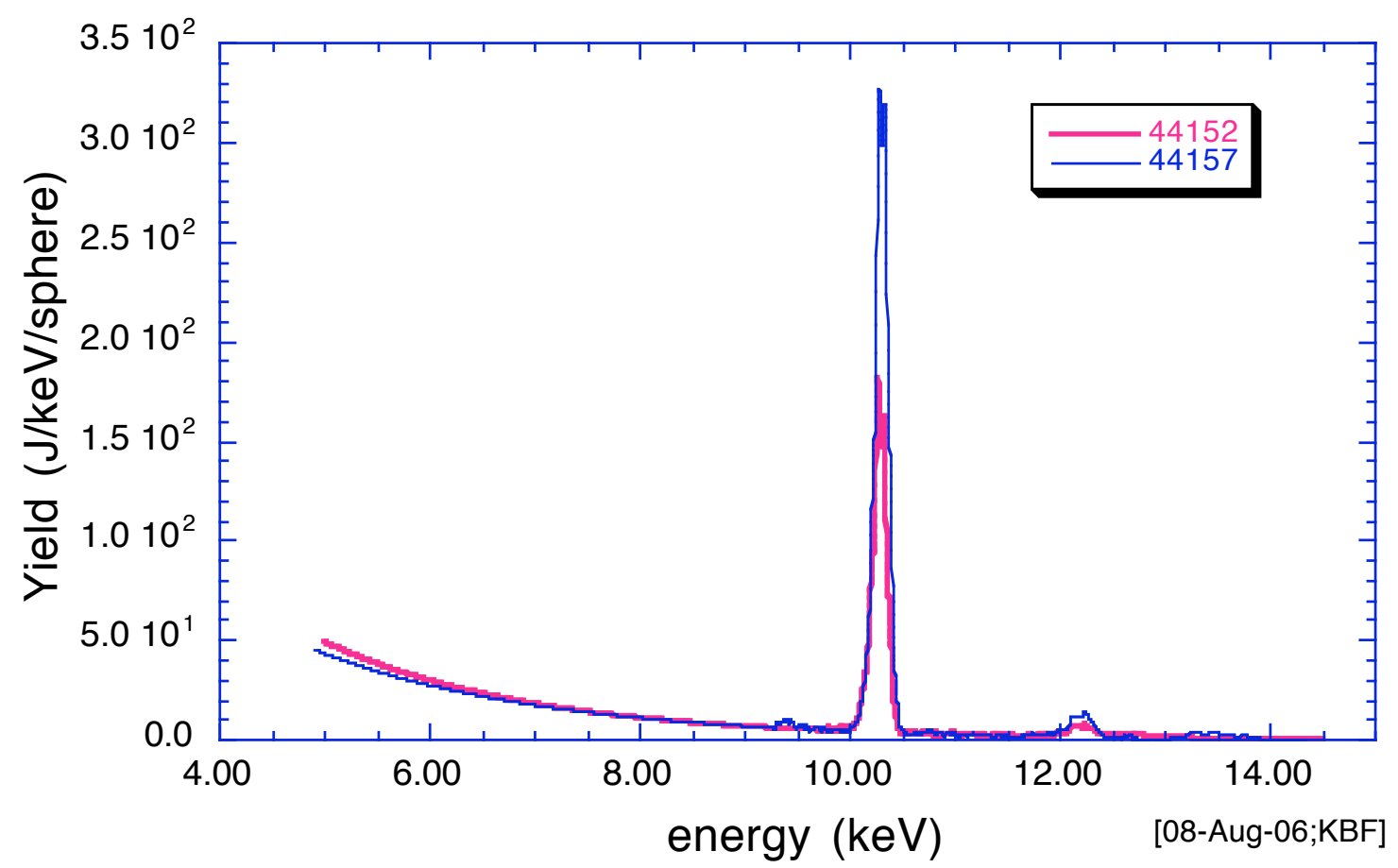

Figure 3 - HENWAY Channel A spectra for shots 44152, 44157 (3 ns, NWET focusing, $3.7 \mathrm{mg} / \mathrm{cm}^{3}, 2.0 \mathrm{~mm} \times 2.2 \mathrm{~mm}$ [ID $\times$ length] $)$. 


\section{1ha_C.sav}

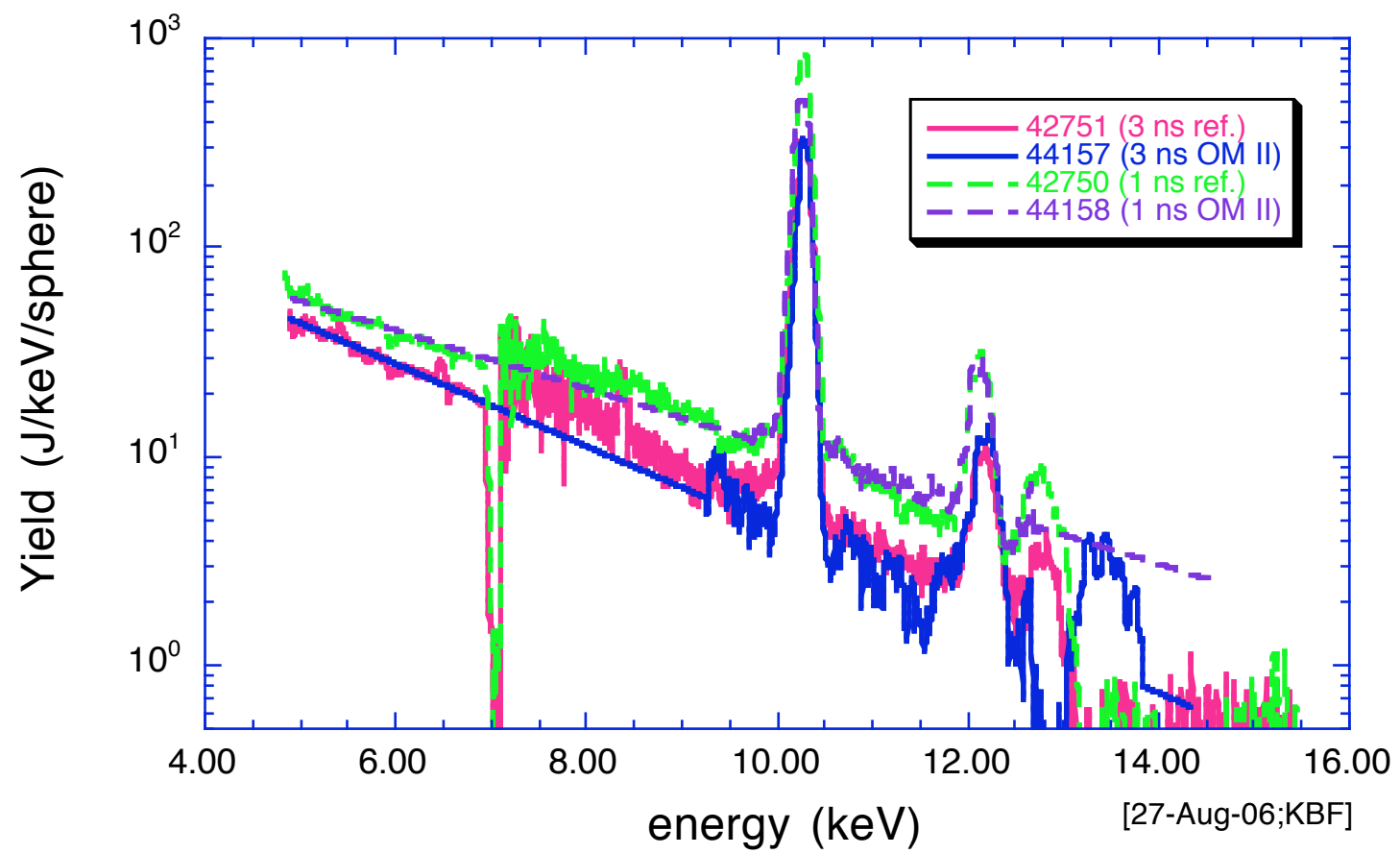

Figure 4 - Comparison of 3-ns (44157) and 1-ns (44158) shot from OMEGA II (7/14/06) with HENWAY Channel A spectra for 42750 and 42751 (our upper-bound pre-shot 'reference' spectra). The difference between the smoothed and inherent continua are obvious. 


\section{2_recommended_spectra}

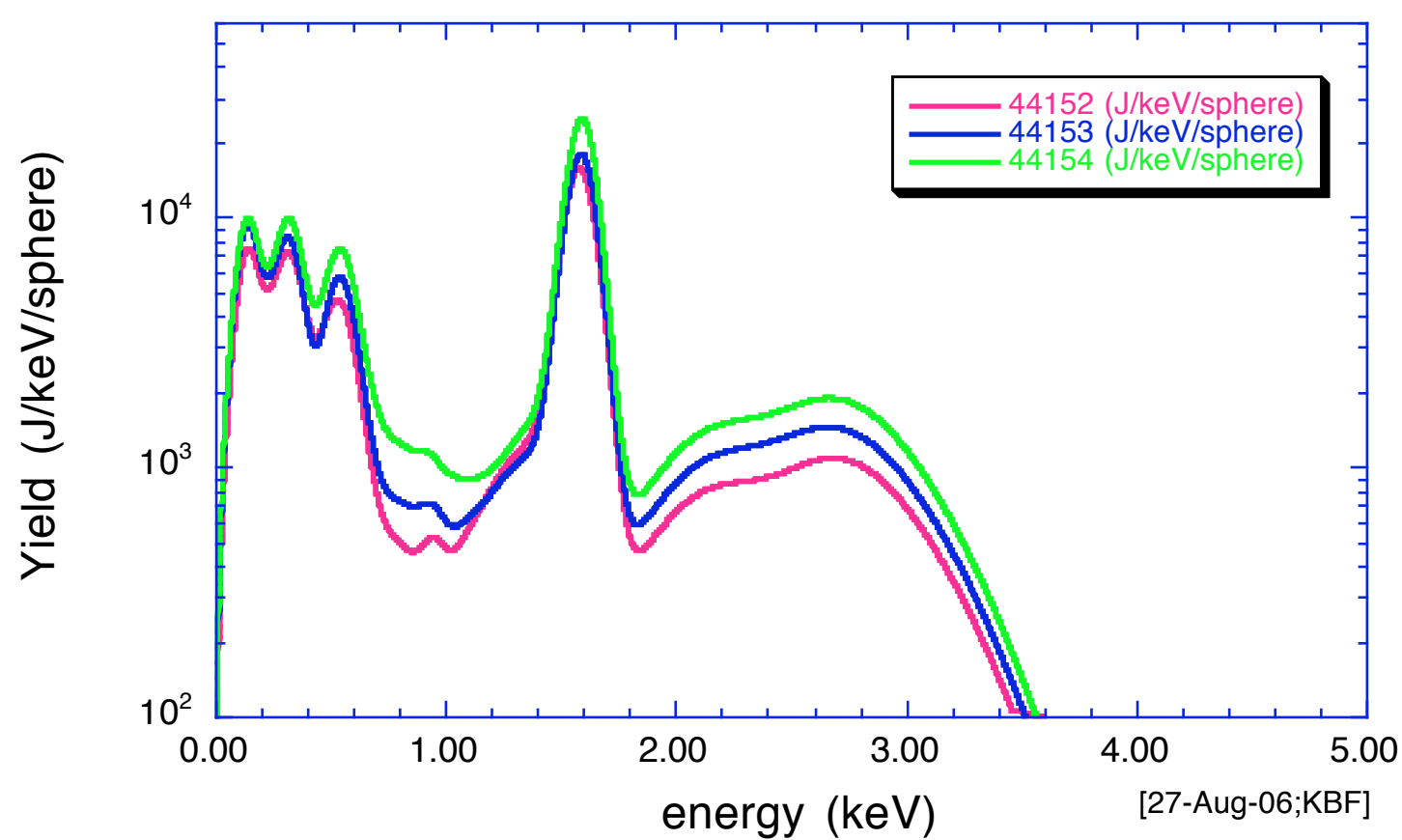

Figure 5 - DANTE spectra for 44152 - 44154. Fluence above $3.5 \mathrm{keV}$ is not accurately represented as we are still trying to understand the response function for the two DANTE high-energy channels (channel 18 and the custom Zr-filtered channel). 


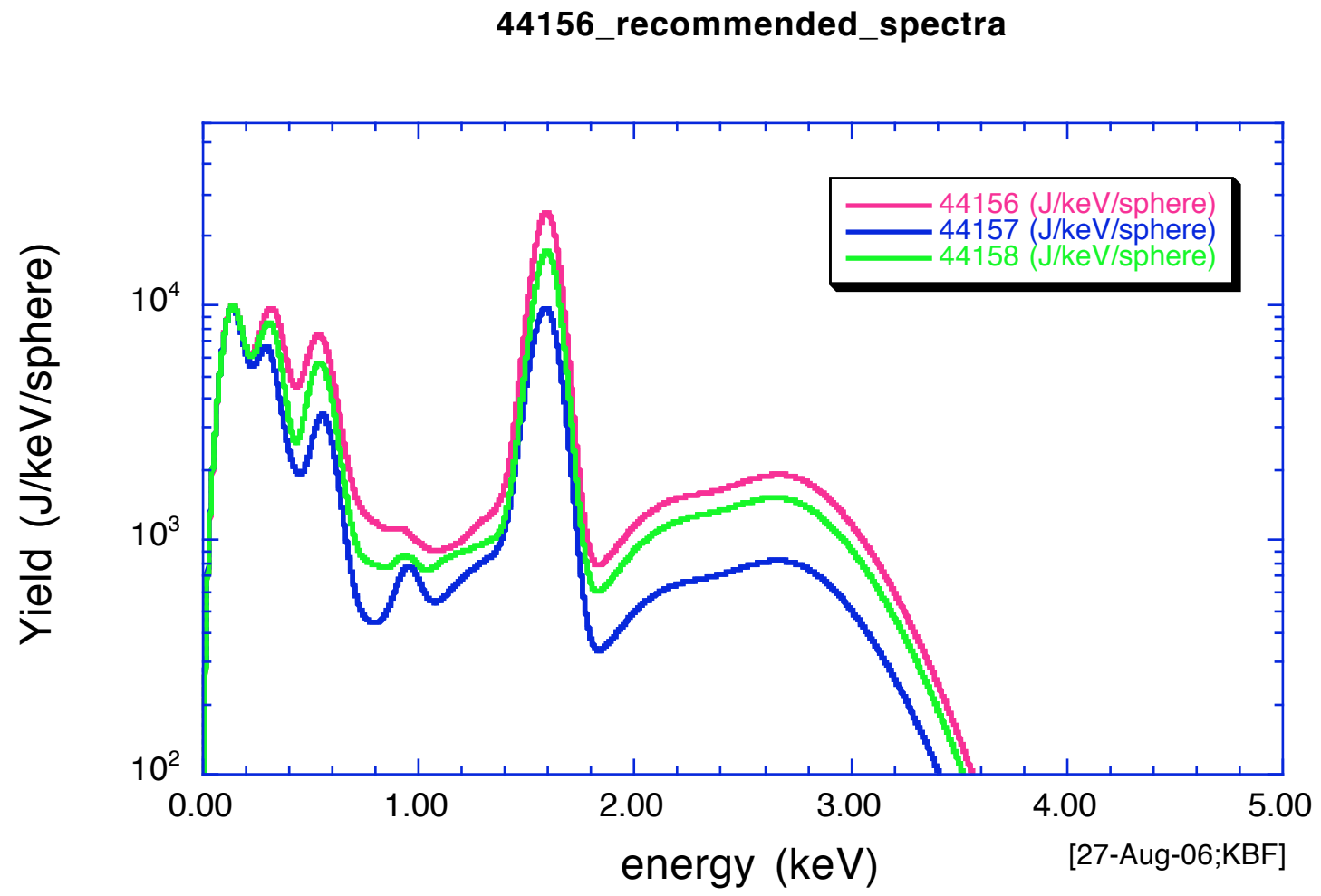

Figure 6 - DANTE spectra for 44152 - 44154. Fluence above $3.5 \mathrm{keV}$ is not accurately represented as we are still trying to understand the response function for the two DANTE high-energy channels (channel 18 and the custom Zr-filtered channel). 


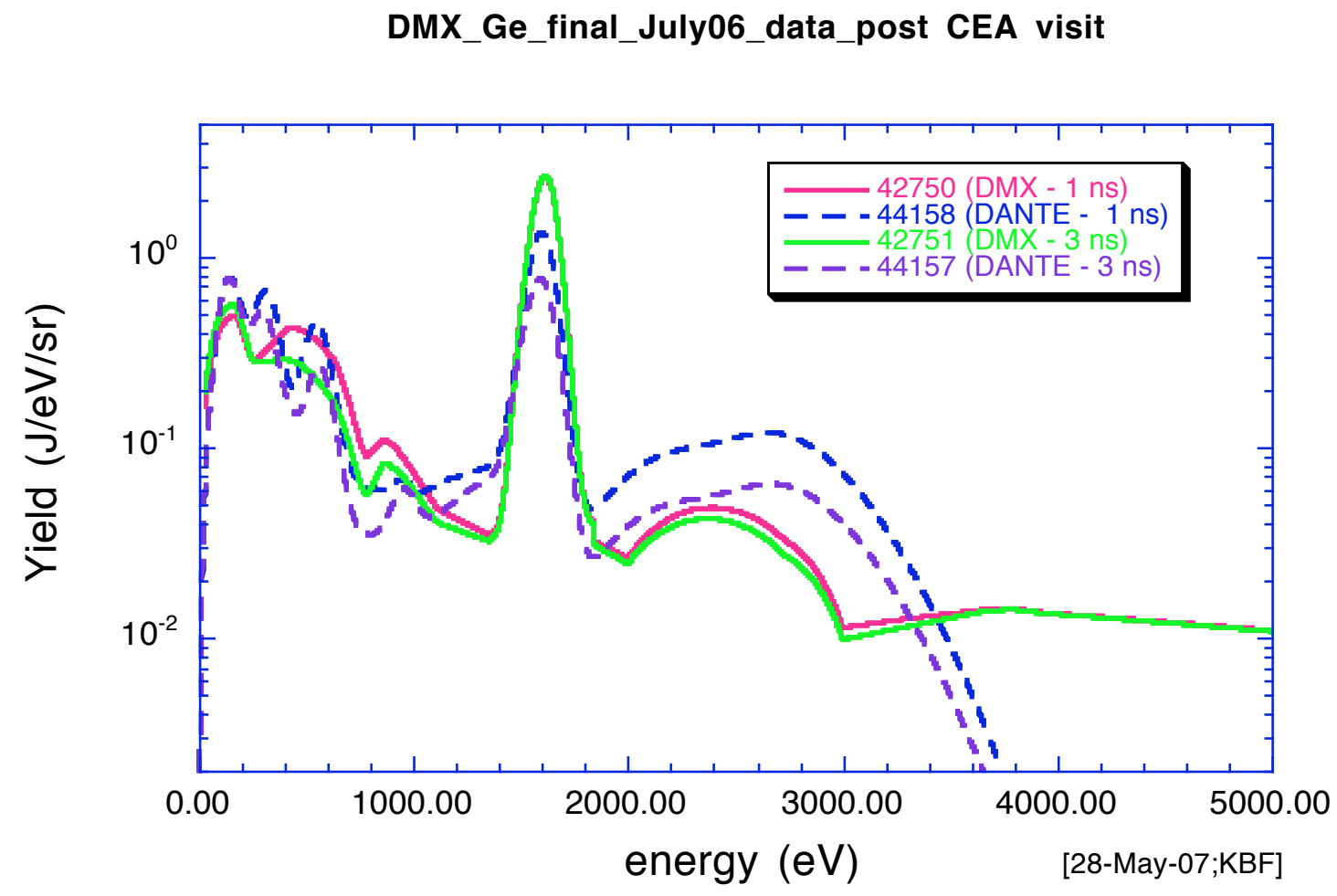

Figure 7 - Comparison of X-ray yields for shots 44158 (1 ns) and 44157 (3 ns) from OMEGA II (7/14/06) measured with DANTE, and the DMX spectra for 42750 (1 ns) and 42751 (3 ns). DMX spectra reflect the final reduction of CEA researchers accomplished October 2006. 


\section{2_recommended_spectra}

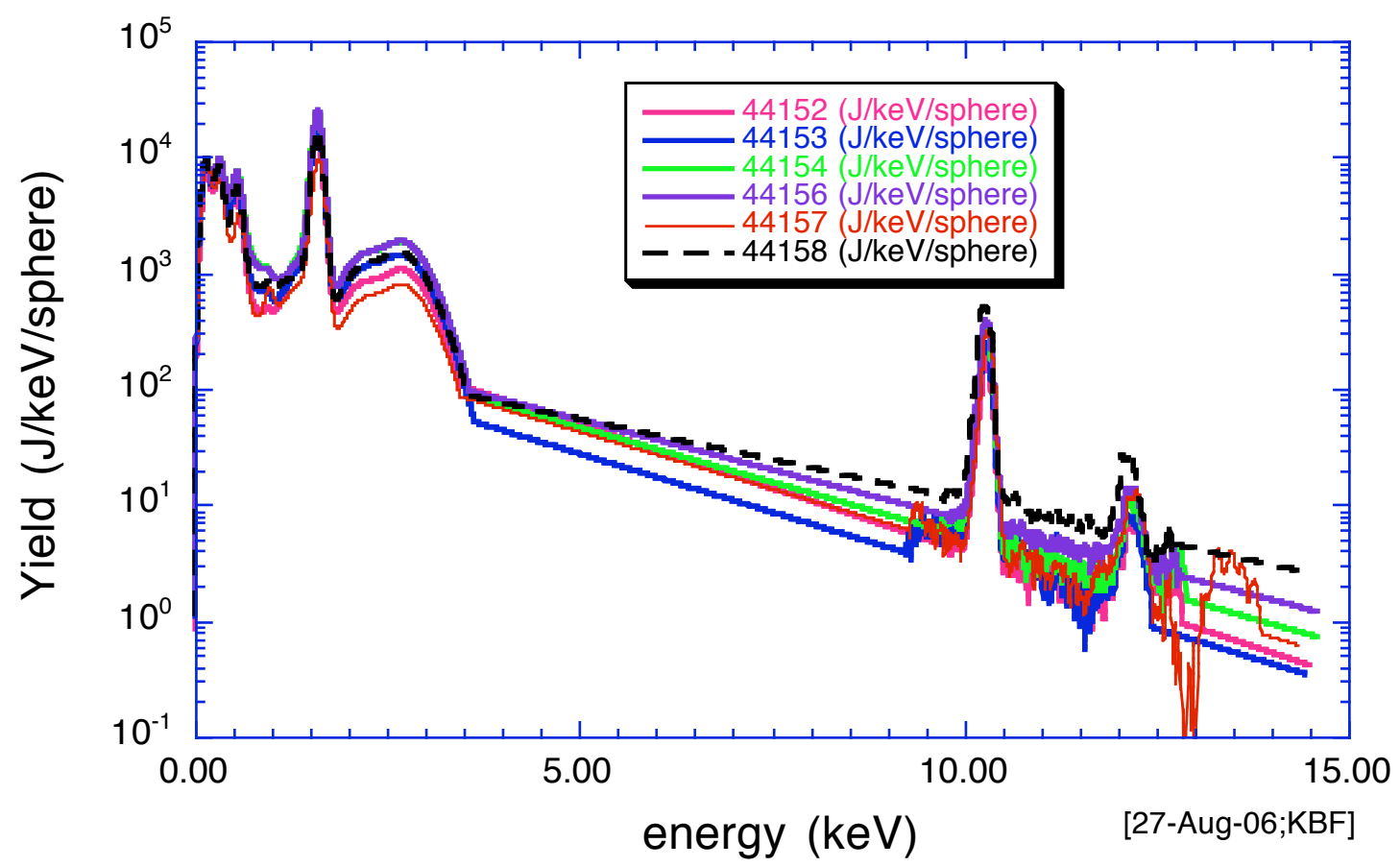

Figure 8 - A summary of the recommended spectra for OMEGA II. 


\section{2data_file}

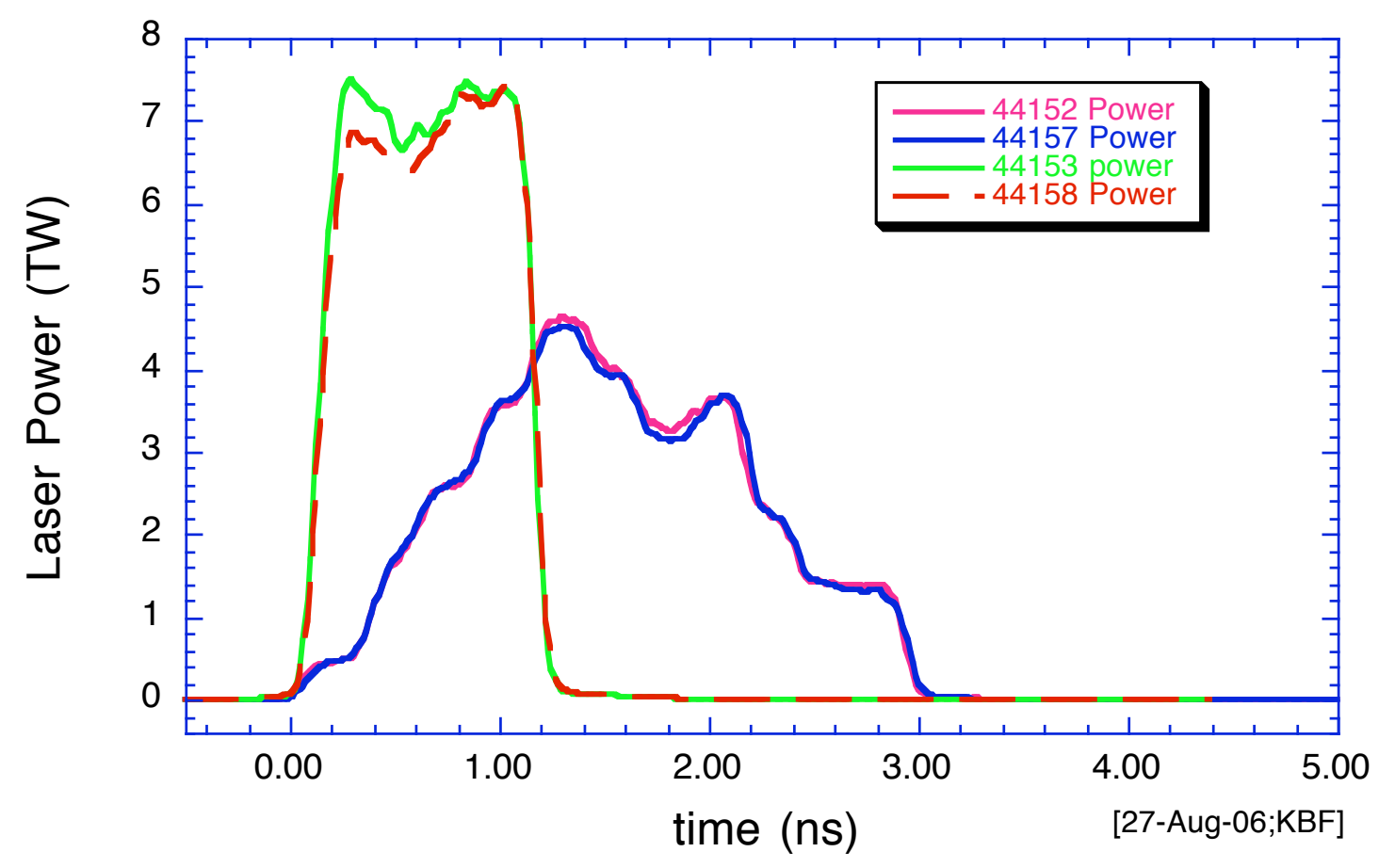

Figure 9 - Laser power delivered to the P8 face of the Ge-doped aerogel target for shots 44152, 44157 ( $3 \mathrm{~ns}$ pulses), 44153 and 44158 ( $1 \mathrm{~ns}$ pulses). The laser power profiles seem quite reproducible. 


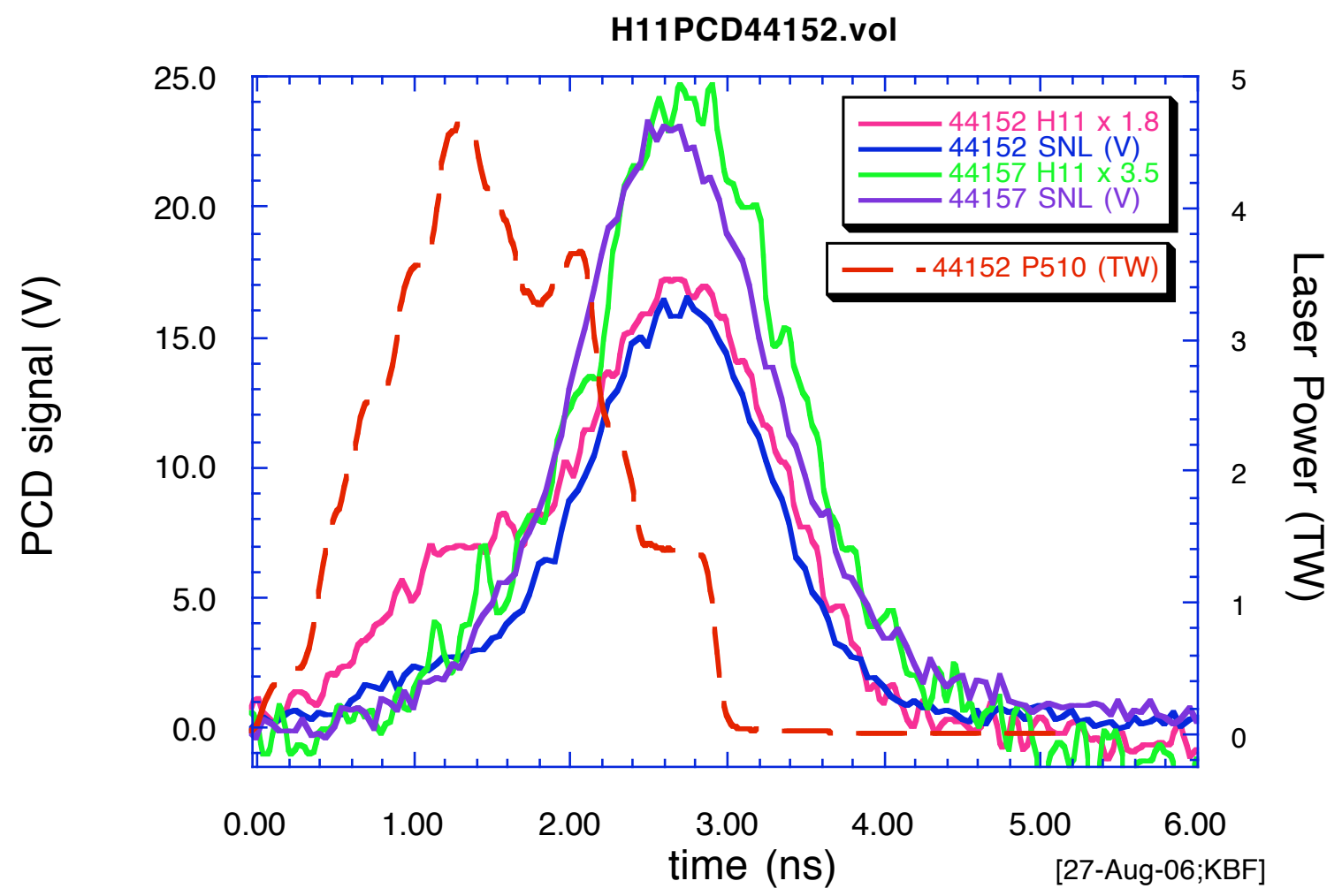

Figure 10 - H11 PCD (filtered with 10 mil Be and $1 / 2$ mil Al, cable corrected) and SNL PCD p126k10 (filtered with 10 mil Kapton and 15 um of Al) X-ray waveforms for 44152 and 44157, shown also with the P510 laser power profile for shot 44152. The H11 voltages have been scaled to be near the SNL PCD voltage, the SNL p126k10 signals have been shifted in time to have the peak voltage correspond to the H11 peak signals. 


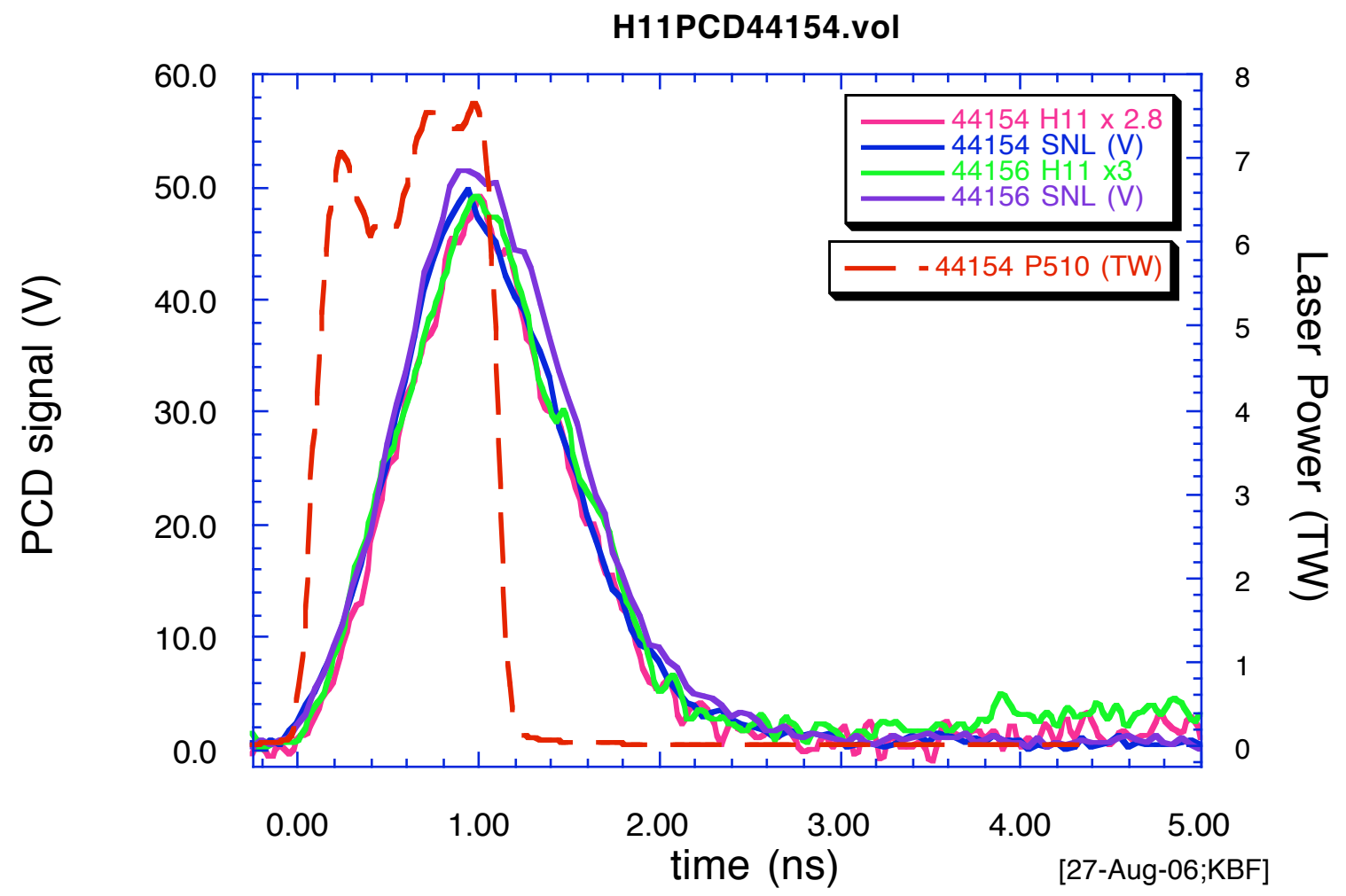

Figure 11 - H11 PCD (filtered with 10 mil Be and $1 / 2$ mil Al, cable corrected) and SNL PCD p126k10 (filtered with 10 mil Kapton and 15 um of Al) X-ray waveforms for 44154 and 44156, shown also with the P510 laser power profile for shot 44154. The H11 voltages have been scaled to be near the SNL PCD voltage, the SNL p126k10 signals have been shifted in time to have the peak voltage correspond to the H11 peak signals. 
H11PCD44153.vol

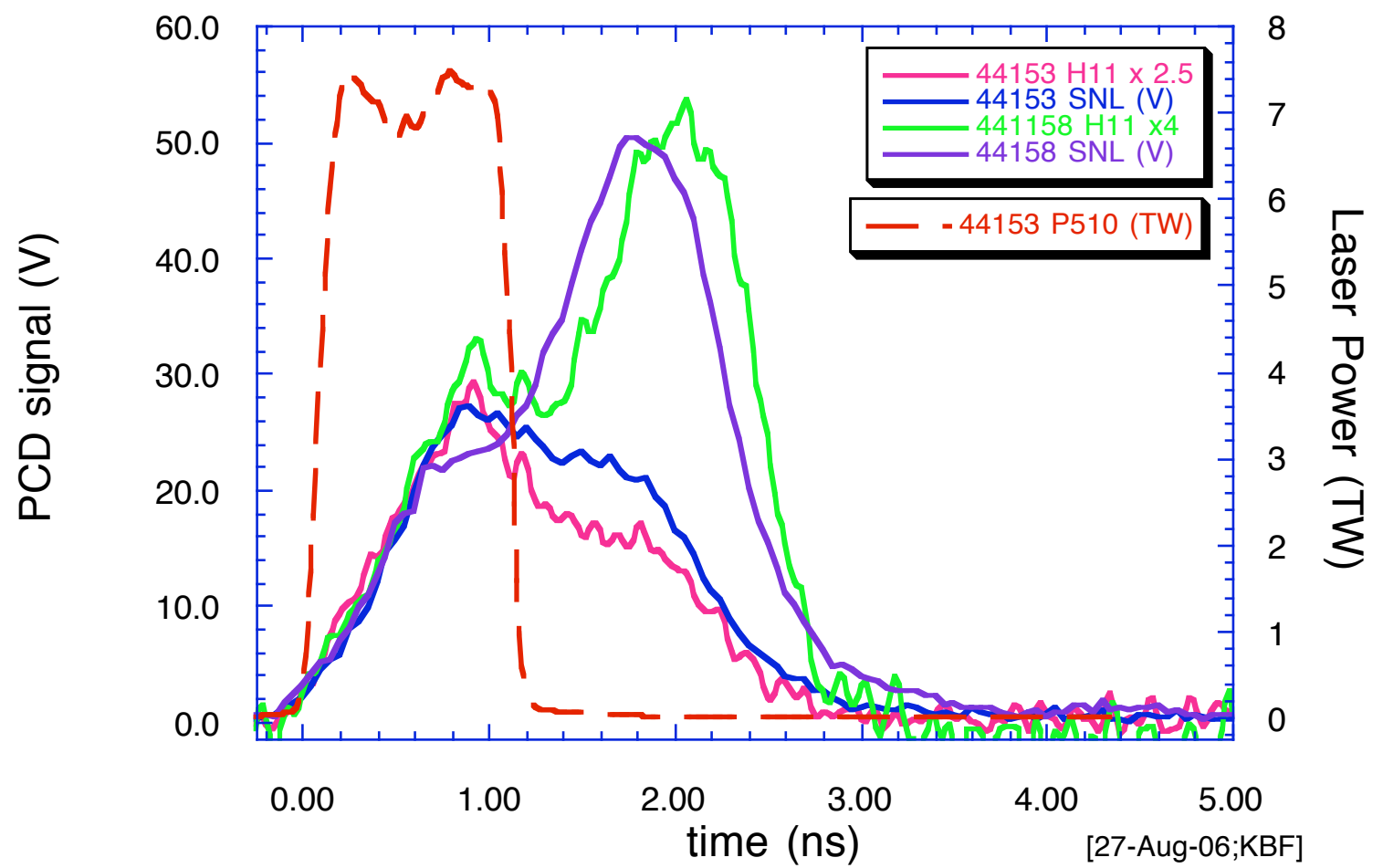

Figure 12 - H11 PCD (filtered with 10 mil Be and $1 / 2$ mil Al, cable corrected) and SNL PCD p126k10 (filtered with 10 mil Kapton and 15 um of Al) X-ray waveforms for 44153 and 44158, shown also with the P510 laser power profile for shot 44153 . The H11 voltages have been scaled to be near the SNL PCD voltage, the SNL p126k10 signals have been shifted in time to have the peak voltage correspond to the H11 peak signals. 


\section{4_p66k10cora}

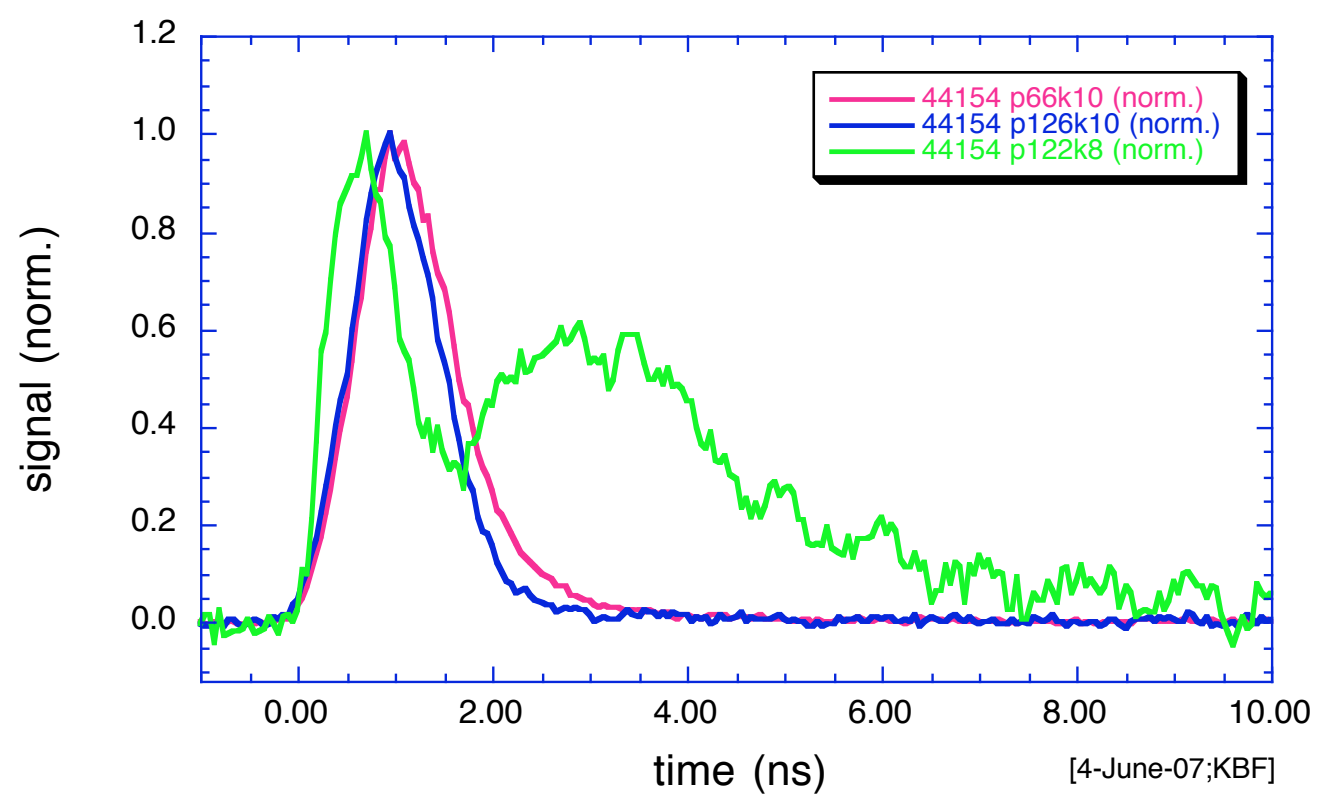

44156_p66k10cora

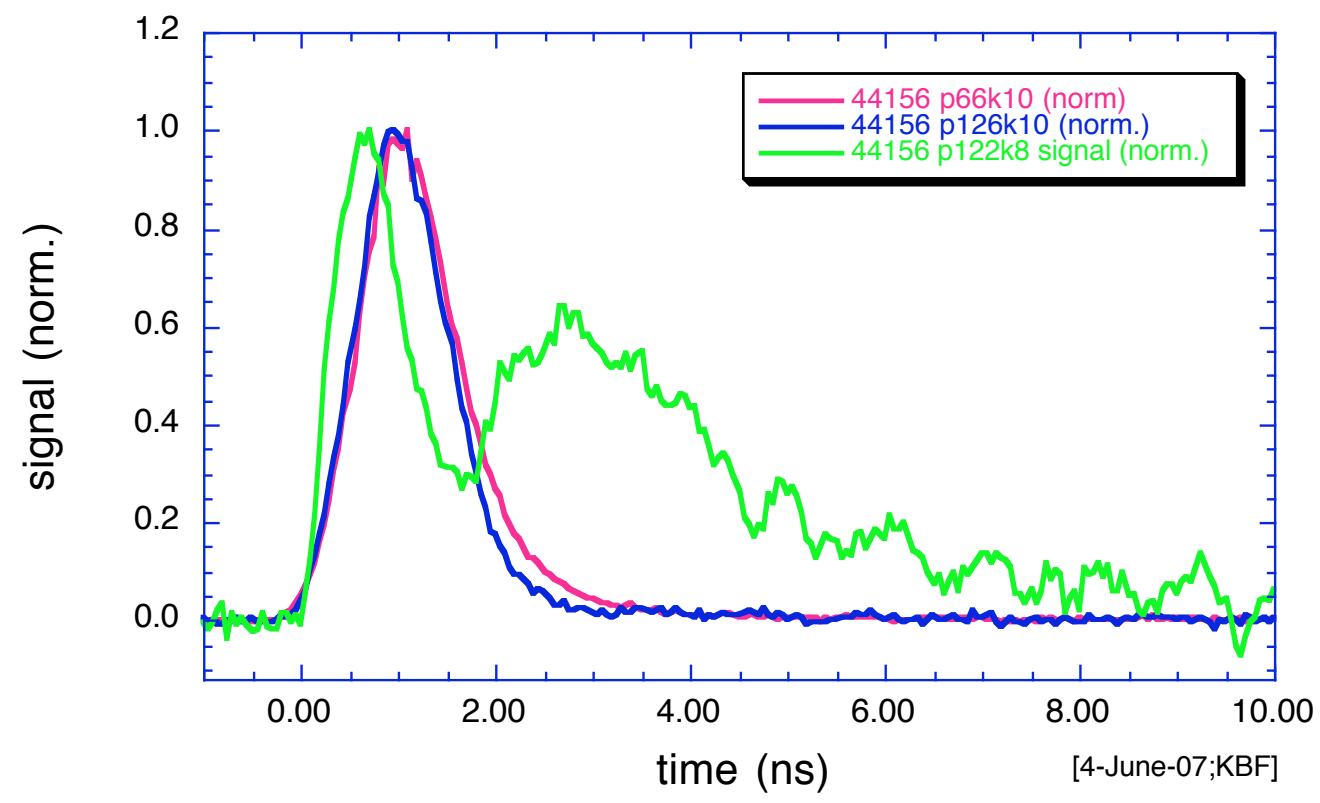

Figure 13 - X-ray signals from three different SNL PCDs (all normalized) on shots 44154 and 44156 (both $1 \mathrm{~ns}$ laser irradiation): p66k and p126k, both filtered with 10 mil of kapton and $15 \mu \mathrm{m}$ of $\mathrm{Al}$, and p122, filtered with $8 \mu \mathrm{m}$ of kapton. The PCDs have an increasing contribution to the measured response from soft and very soft X-ray photons that results in the progressively broader X-ray waveforms. 
44153_p122k8cora

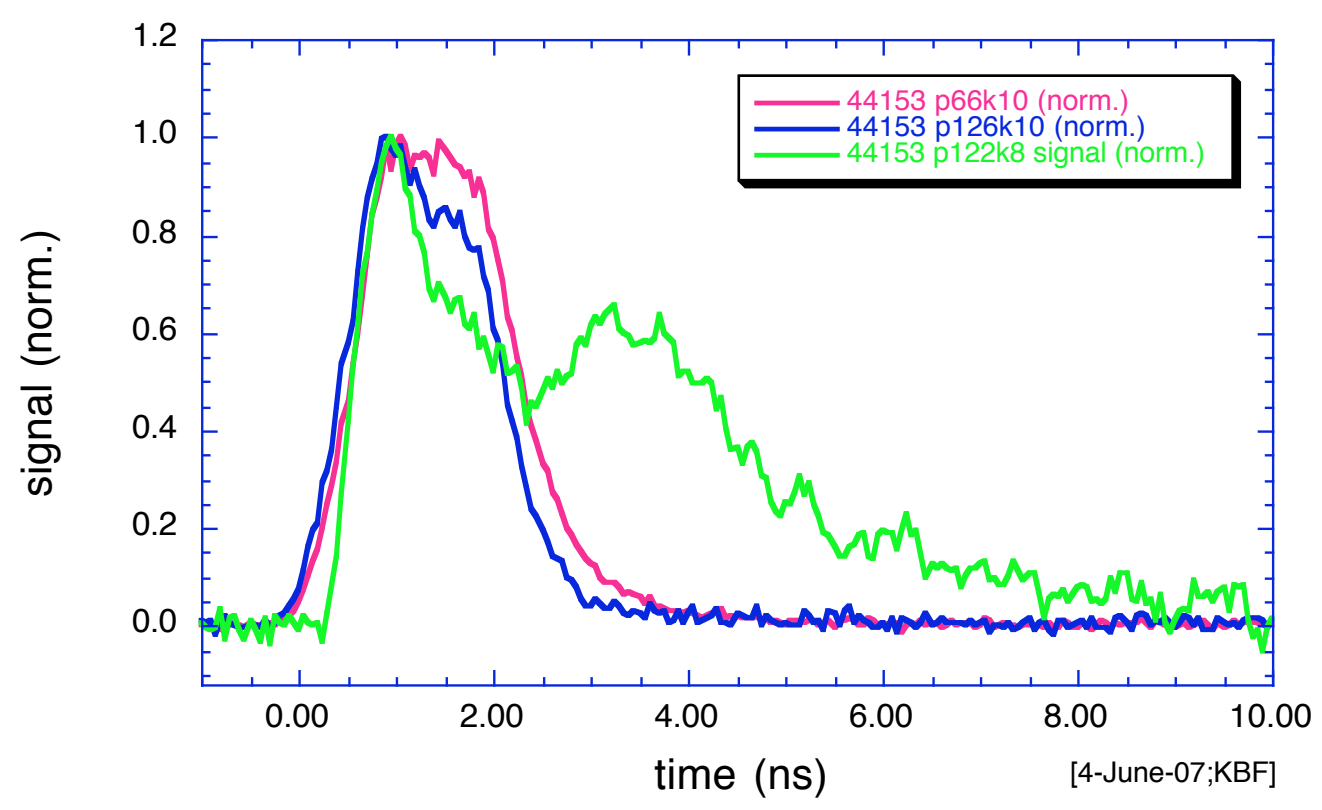

44158_p66k10cora

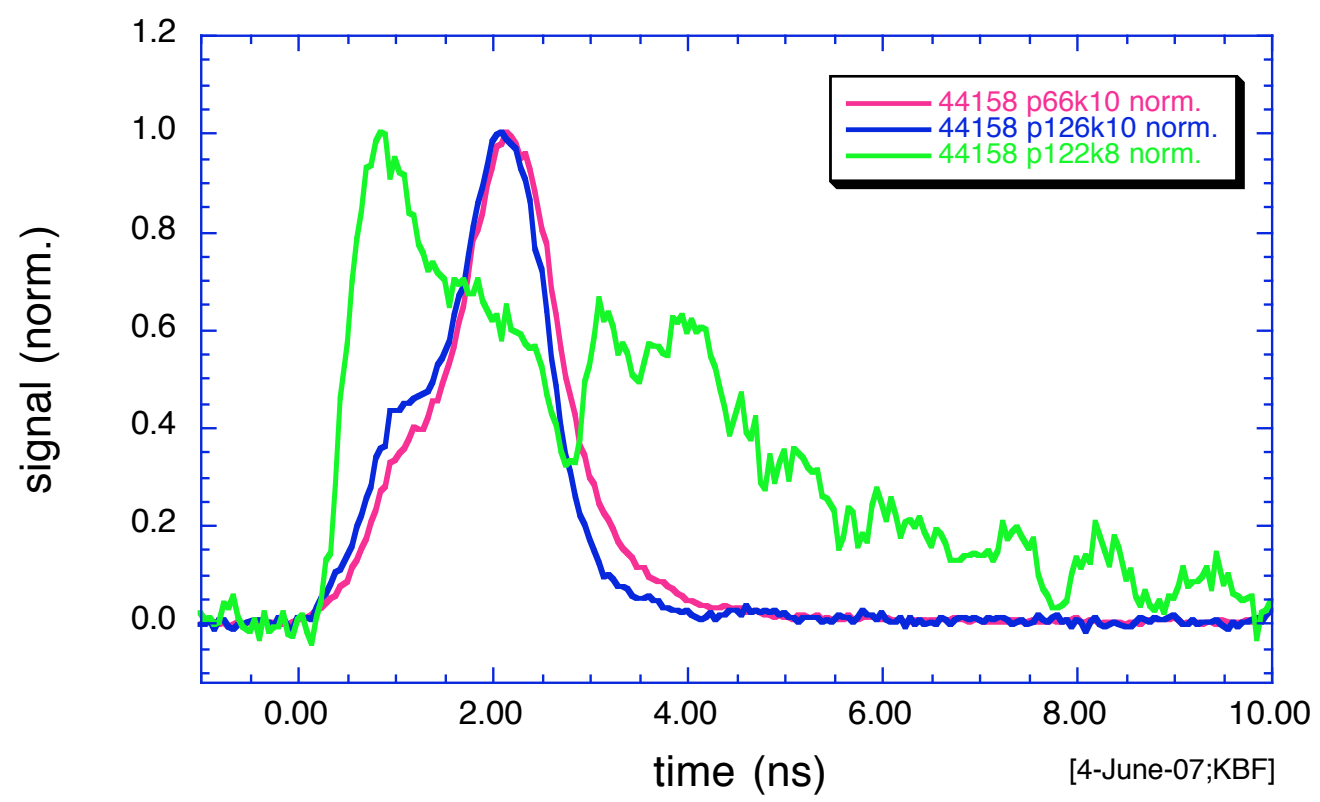

Figure 14 - X-ray signals from three different SNL PCDs (all normalized) on shots 44153 and 44158 (both $1 \mathrm{~ns}$ laser irradiation): p66k and p126k, both filtered with 10 mil of kapton and $15 \mu \mathrm{m}$ of $\mathrm{Al}$, and p122, filtered with $8 \mu \mathrm{m}$ of kapton. The PCDs have an increasing contribution to the measured response from soft and very soft X-ray photons that results in the progressively broader X-ray waveforms. These two shots have unusual $\mathrm{X}$-ray waveforms. 
44152_p66k10cora

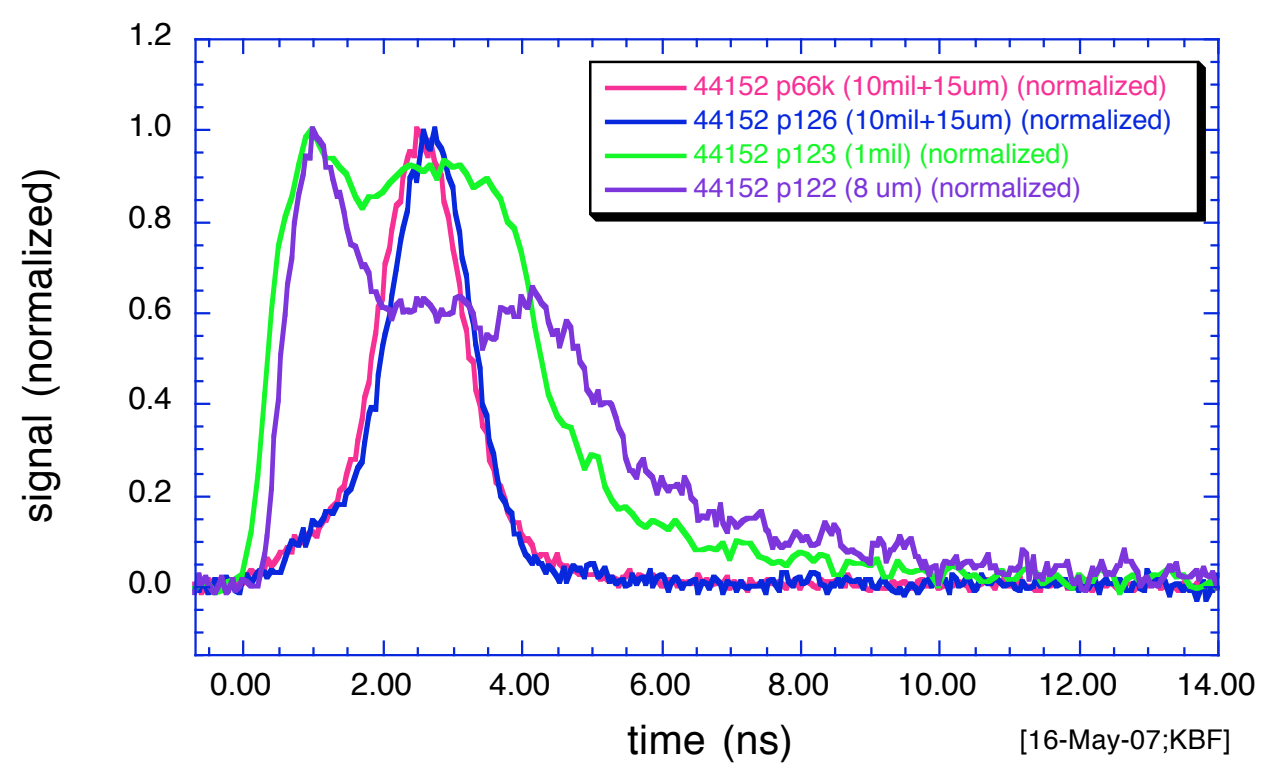

44157_p66k10cora

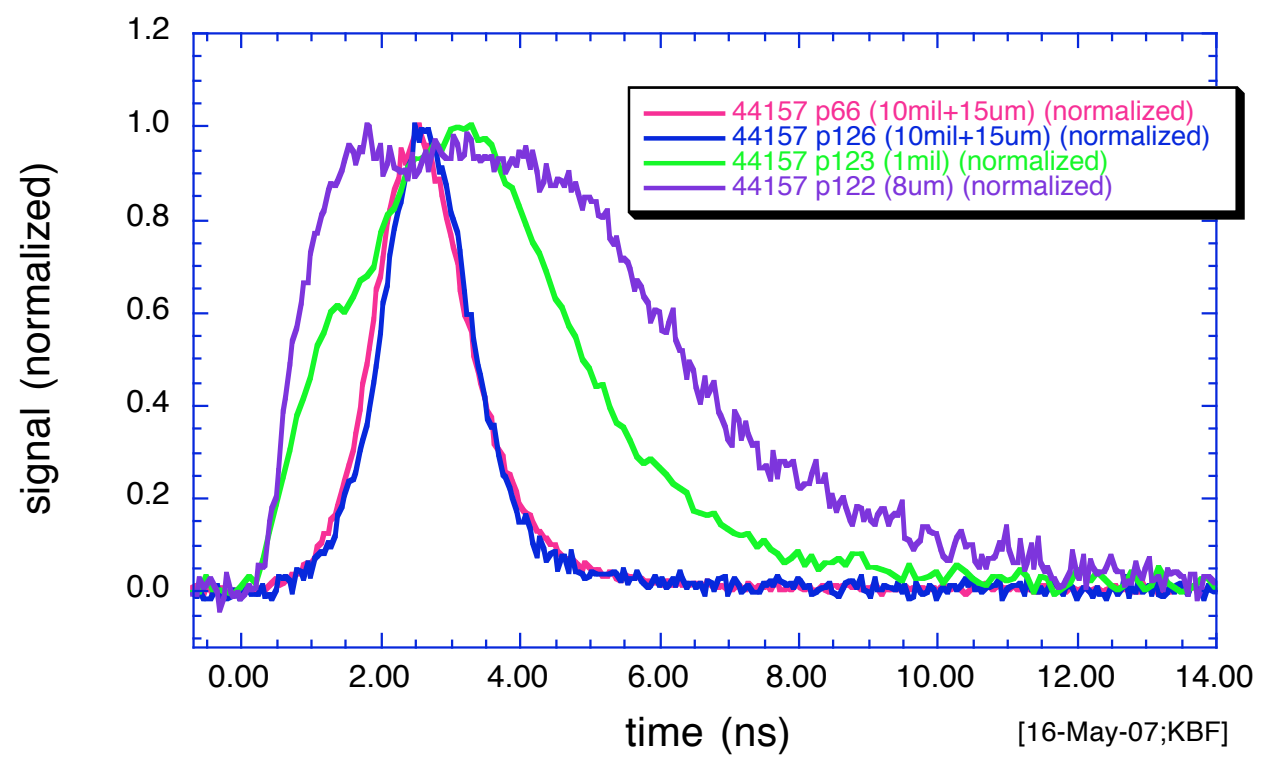

Figure 15 - X-ray signals from four different SNL PCDs (all normalized) on shots 44152 and 44157 (both 3 ns laser irradiation): p66k and p126k, both filtered with 10 mil of kapton and $15 \mu \mathrm{m}$ of Al, p123, filtered with 1 mil kapton, and p122, filtered with $8 \mu \mathrm{m}$ of kapton. The PCDs have an increasing contribution to the measured response from soft and very soft X-ray photons that results in the progressively broader X-ray waveforms. 
44152_p123k1cora 15:58:38 5/17/07

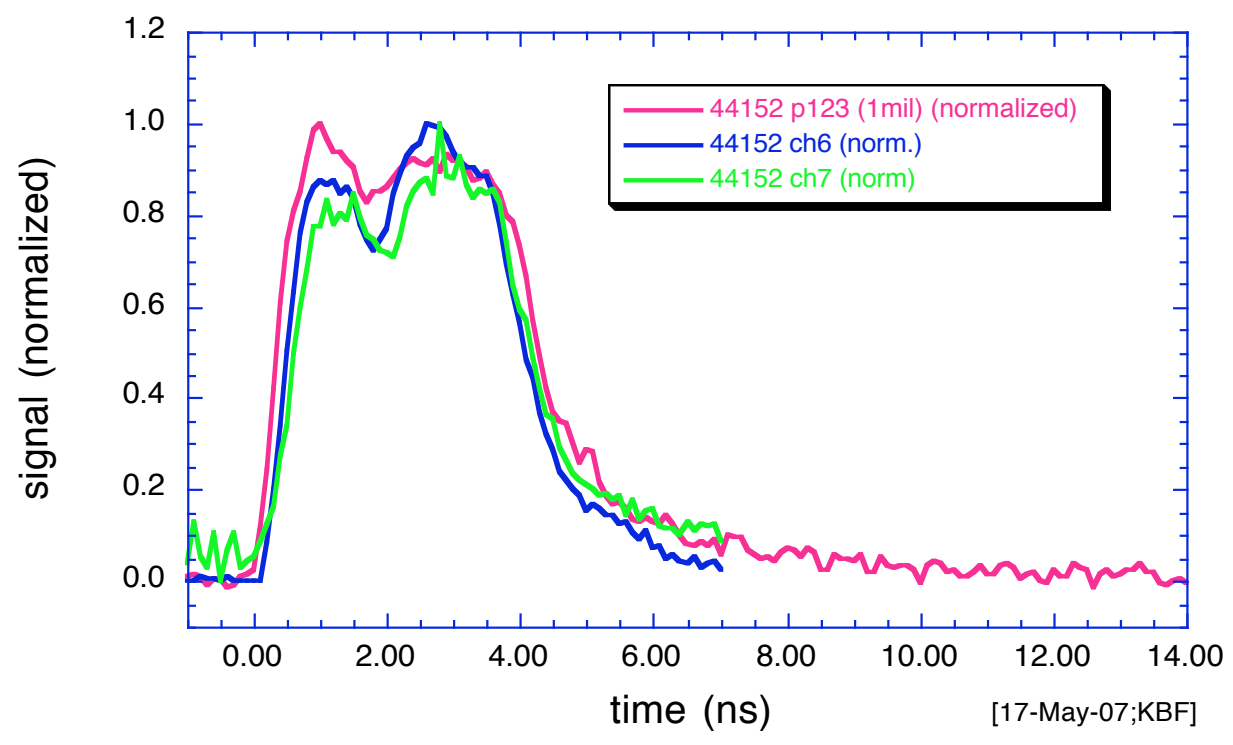

44152_p122k8cora

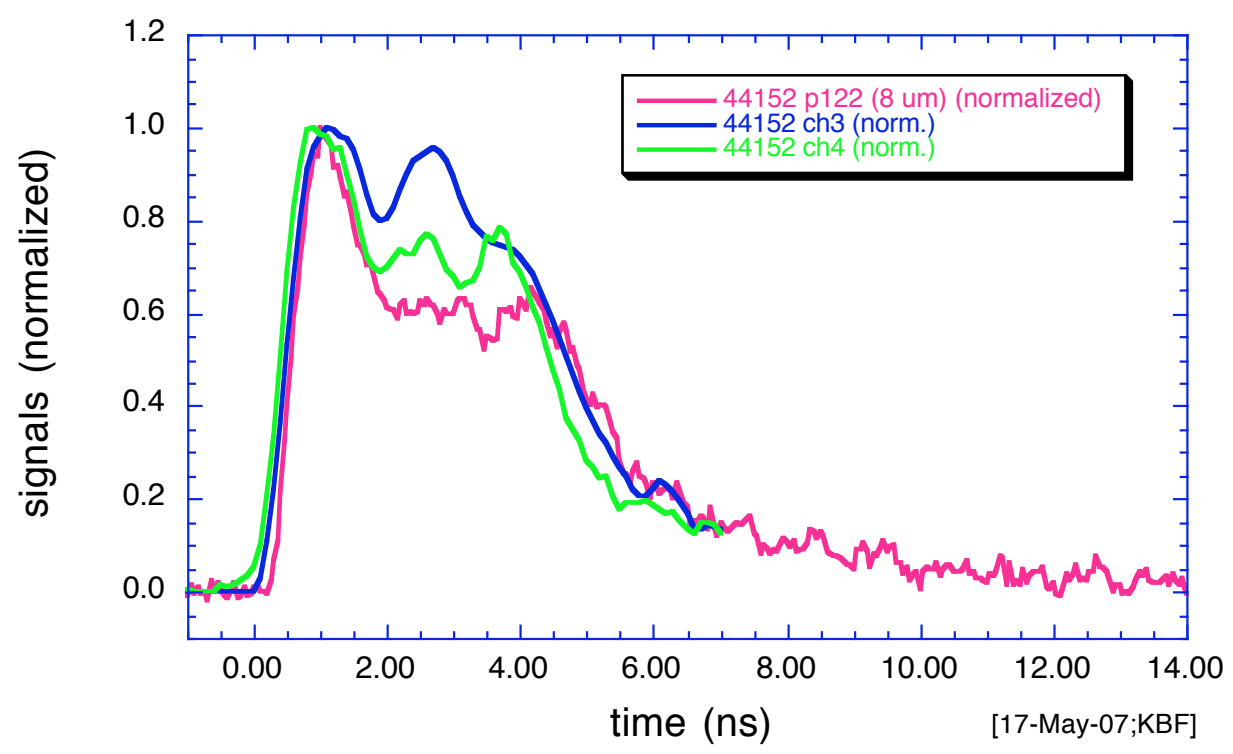

Figure 16 - (top) SNL PCD response for p123 (filtered with 1 mil kapton) and the response of DANTE channels 6 and 7 (band passes of $0.9-1.05 \mathrm{keV}$ ); the signals are substantially broader than those of the harder X-ray responses. (bottom) SNL PCD p122 (filtered with 8 um Kapton) and two softer DANTE channels (channels 3 and 4, band passes $0.25-0.28$ and $0.45-0.52 \mathrm{keV}$, respectively). The $\mathrm{X}$-ray responses get continually broader as softer photon emission is included in the measured signals. There is good consistency between the SNL PCD and H11 and DANTE XRD signals. 
44157_p123k1cora 16:05:11 5/17/07

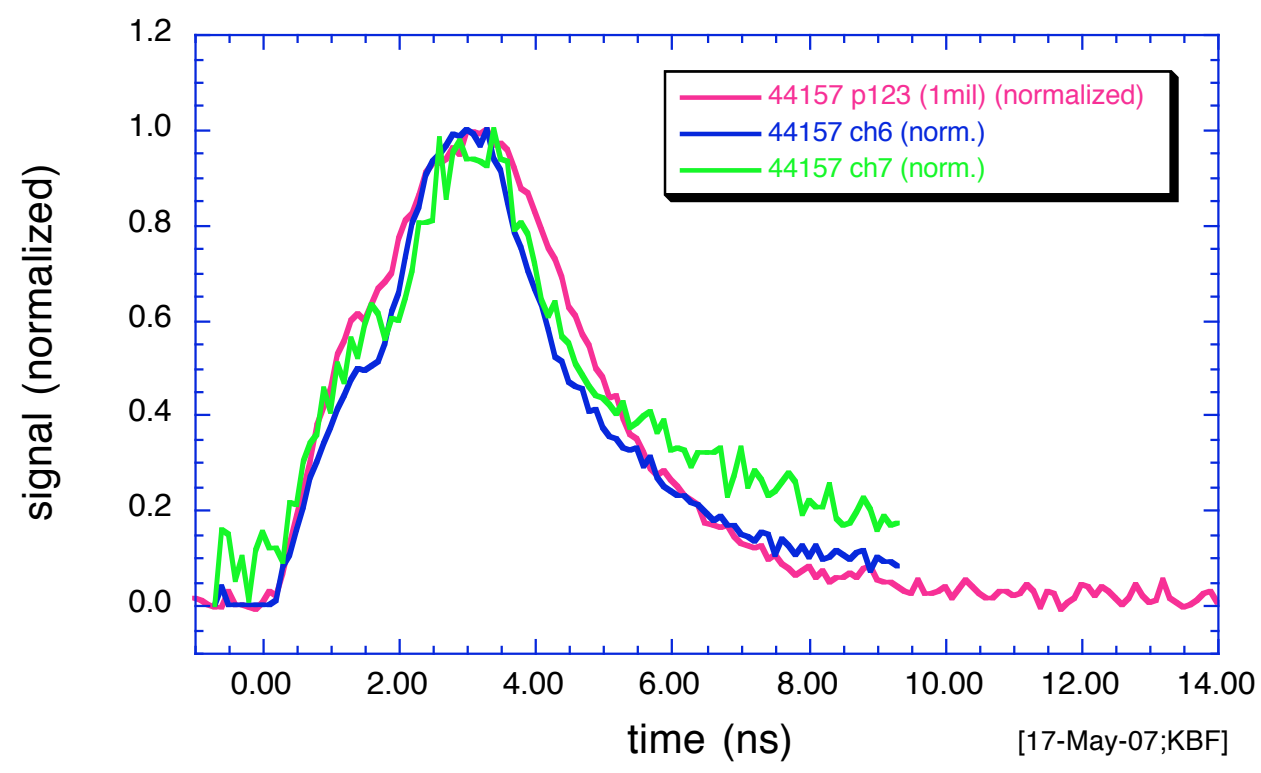

44157_p122k8cora

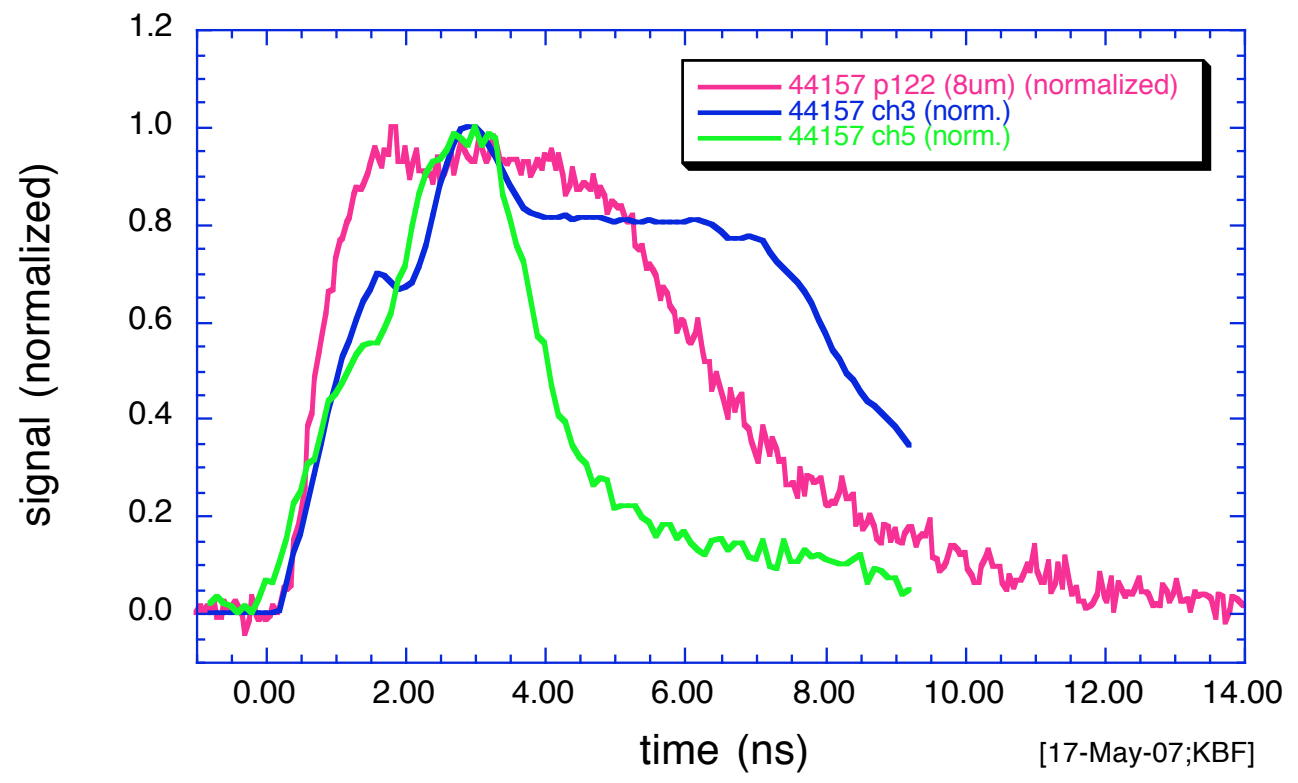

Figure 17 - (top, bottom) Data from shot 44157, as in Figure 16, except it is DANTE channels 3 and 5 that are compared to the p122 PCD signal (DANTE channel 4 did not return usable data - bandpass for DANTE channel 5 is $0.75-0.82 \mathrm{keV}$ ). The shape of the soft X-ray response in shot 44157 is unusual, bordering on pathological. There is good consistency between the SNL PCD and DANTE XRD signals for the middle panel; the two displayed DANTE channels bracket the p122 signal in the bottom panel. 


\section{Appendix A}

Here are questions from Christine Coverdale to KBF, and answers that contain content from Mark May and Steve MacLaren (LLNL) and Chuck Sorce (LLNL and LLE).

1. What do the raw spectral data look like? You indicate that it is noisy near the Fe edge.... Just wondering how it looks relative to what we had from Omega I.

Here are the three spectra from OMEGA I before the introduction of the Fe filter (Fig. A.1): note how smooth the continuum is on a $\log$ scale, except for that "bump" at the edge of the crystal/film near $4.7 \mathrm{keV}$. Also, see the noise above the $\mathrm{He}_{\gamma}$ feature near 13 keV. I have "smoothed" spectra from OMEGA I now (like those I sent around for OMEGA II), and, as soon as I correct the signal levels from the softer-energy HENWAY channel (KAP crystal), I'll distribute the "smoothed" OMEGA I .

\section{2ha_C.sav}

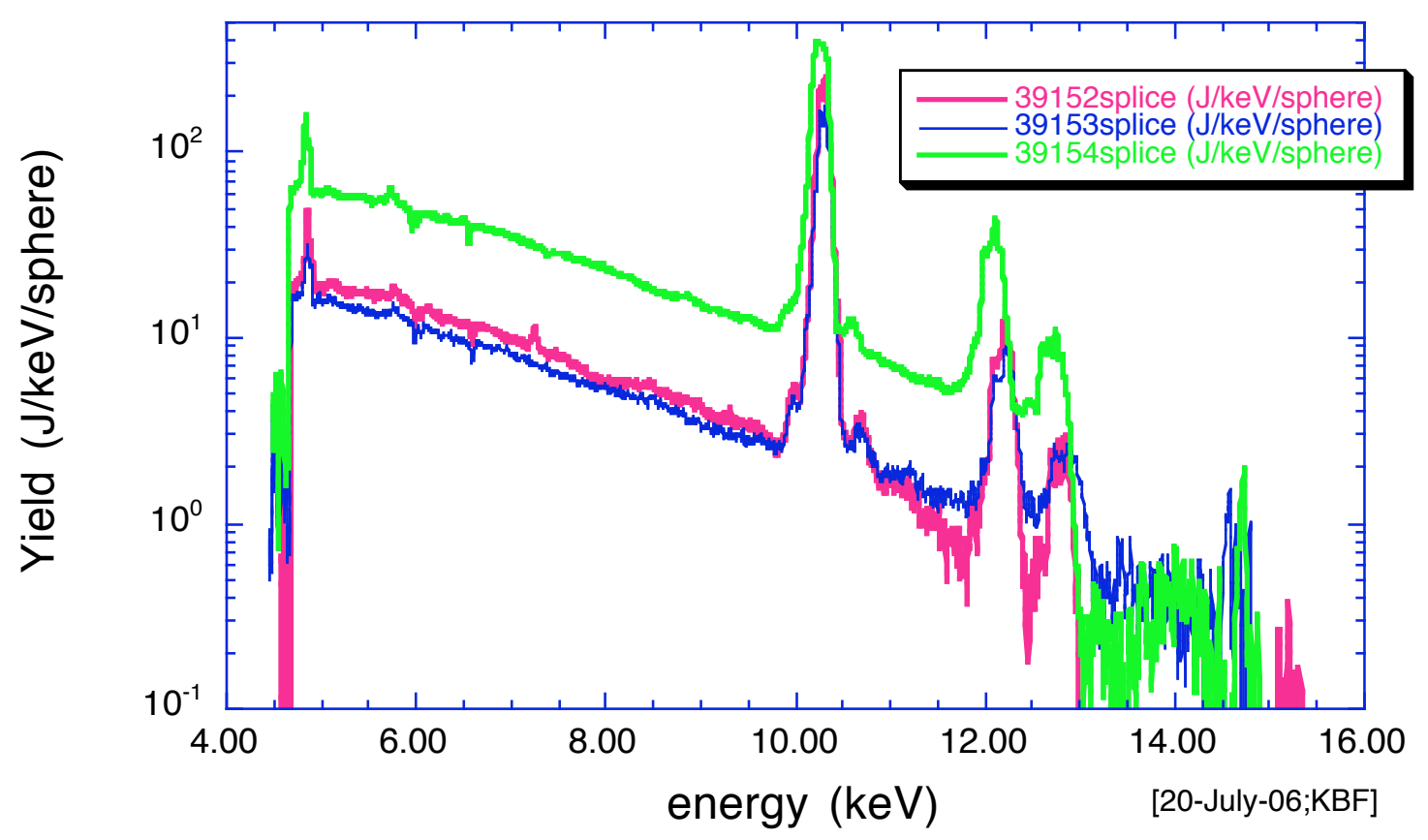

Fig. A.1 - HENWAY spectra (PET crystal) from shots 39152 - 4. From reanalysis done on $07 / 20 / 2006$.

Now, Fig. A.2 shows the three spectra from the HENWAY PET channel after the Fe foil was added to the filter pack: You can see how noisy the data are above the Fe Kedge. Yes, I see that there is a shift in the energy of the lines between 39156-7 and 39158. I calibrated the first two on the $\mathrm{He}_{\alpha}$ line at $10.2806 \mathrm{keV}$, while I calibrated the third one on the Fe K-edge at $7.112 \mathrm{keV}$, all of which are obvious in the images of the film that are in appendix $\mathrm{C}$ of this report. Note, when I calibrate on the Fe K-edge, there 
is no dip going to zero in the signal level (as you see in the blue and pink traces), but there is a big spike just above the edge. Clearly, I need to tweak the dispersion model (and re-examine what I have for the crystal geometry and the source-to-spectrometer distances in the IDL code I use to calibrate the scanned film images).

\section{6ha_C.sav}

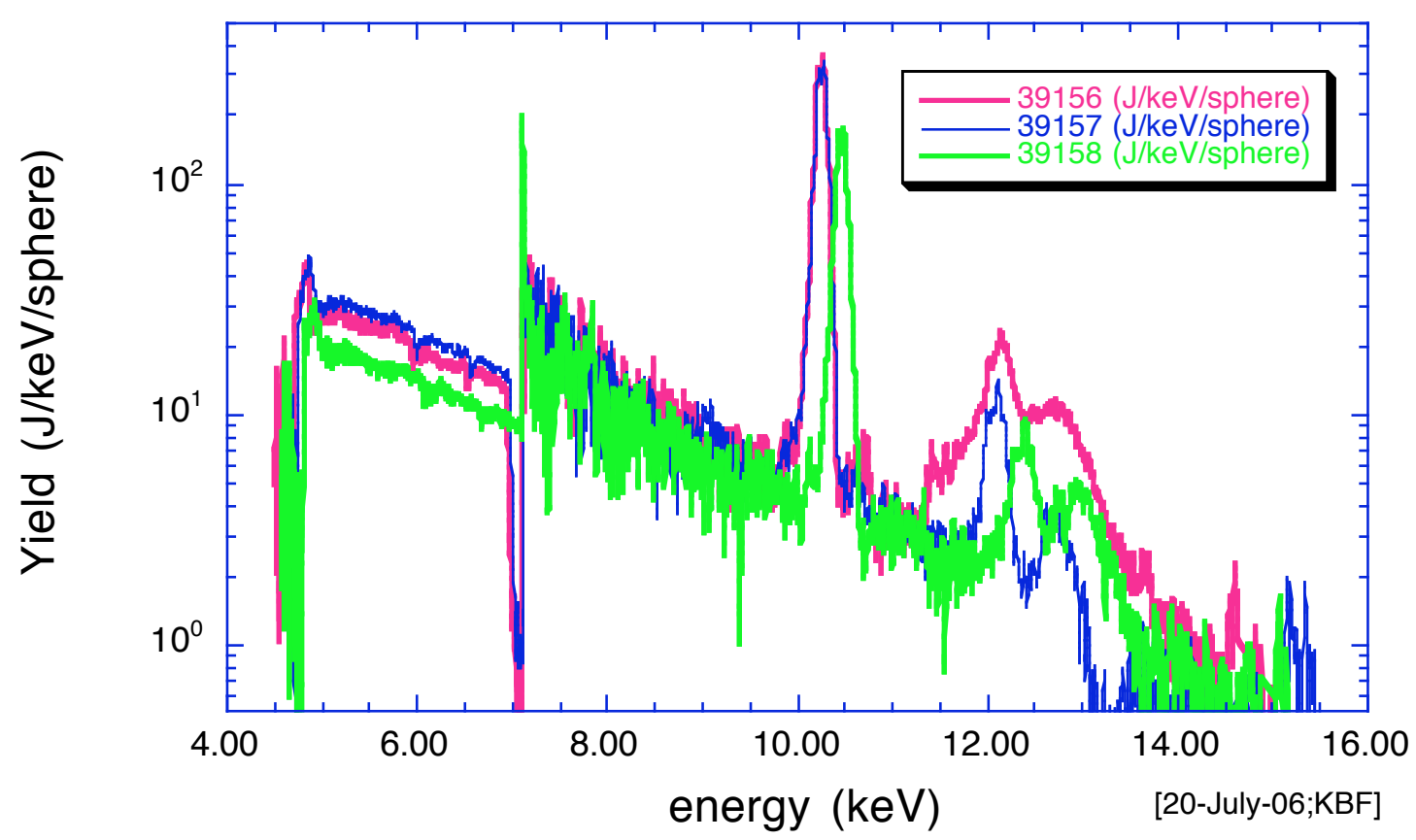

Fig. A.2 - HENWAY spectra (PET crystal) from shots 39156 - 8. From reanalysis done on $07 / 20 / 2006$.

Figure A.3 shows you the raw data from the PET channel in HENWAY for the "1 ns" shots in OMEGA II. It's not too bad, but since I think Tom has had a problem reconciling the measured to simulated ratios for the data with the $\mathrm{Fe}$ foils from OMEGA I, I thought to move to the "smoothed", highly massaged data. 
07/14/06 - 1 ns Ge K-shell spectra

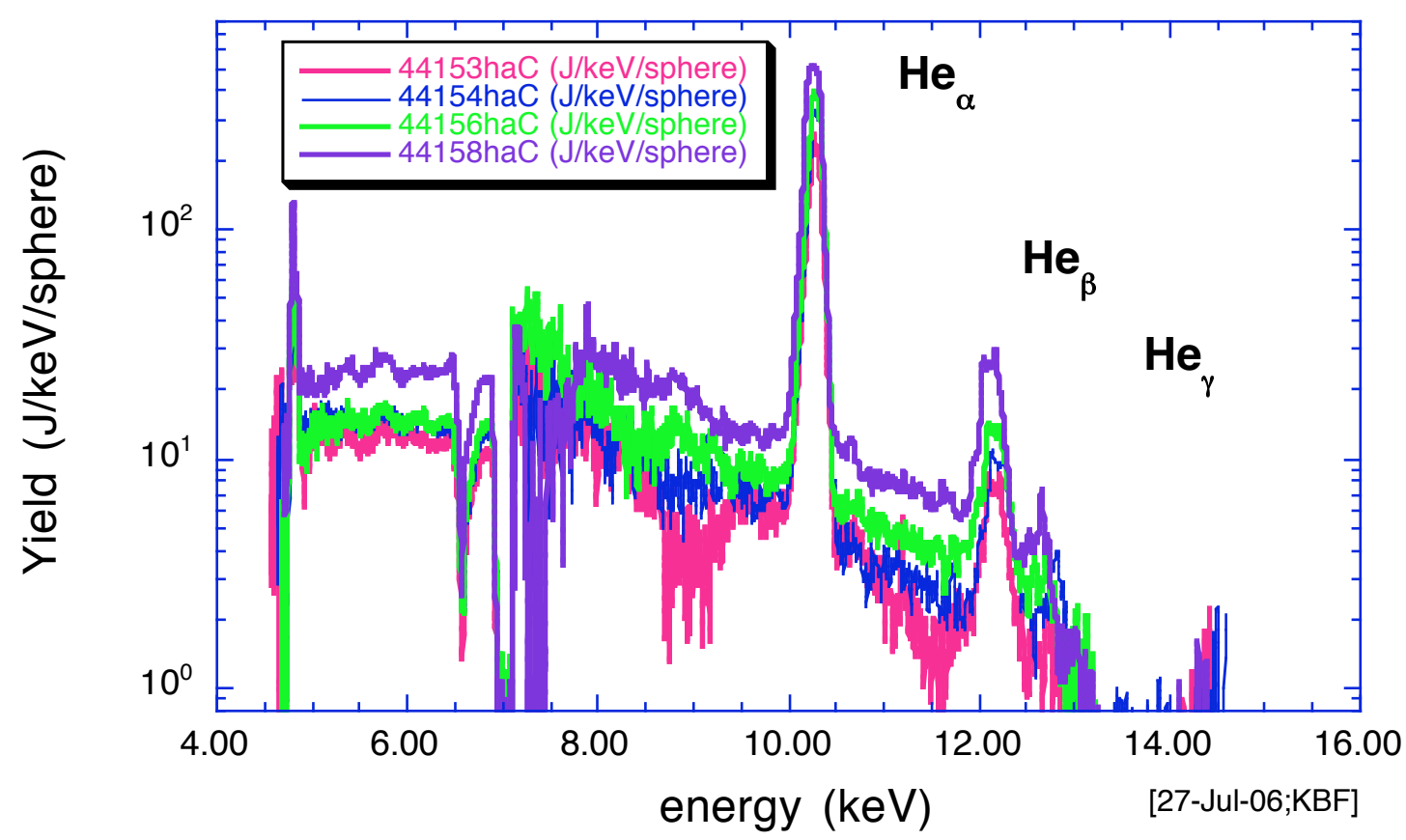

Fig. A.3 - Pre-smoothing data from the PET-crystal channel in HENWAY for OMEGA II.

For completeness sake, Figure A.4 shows you the data for the two (inappropriately named) "3 ns" shots. They look, pretty much just like the spectra in Fig. A.3. Note, if you look at the film images in Appendix C, you'll see different regions of different film density on each piece of film. In addition to the Fe and Ti foils, there was $\mathrm{Al}$, and different thicknesses of Be over different parts of the film. If I take cuts through the different parts of the filter pack, I get answers for the yields that are consistent to better than $10 \%$, which indicates that the filter thicknesses are what we believe them to be.

As stated in Section II of the report above, we believe the yields in any band from the unfolded HENWAY have a $\pm 25 \%$ uncertainty. 
07/14/06 - 3 ns Ge K-shell spectra

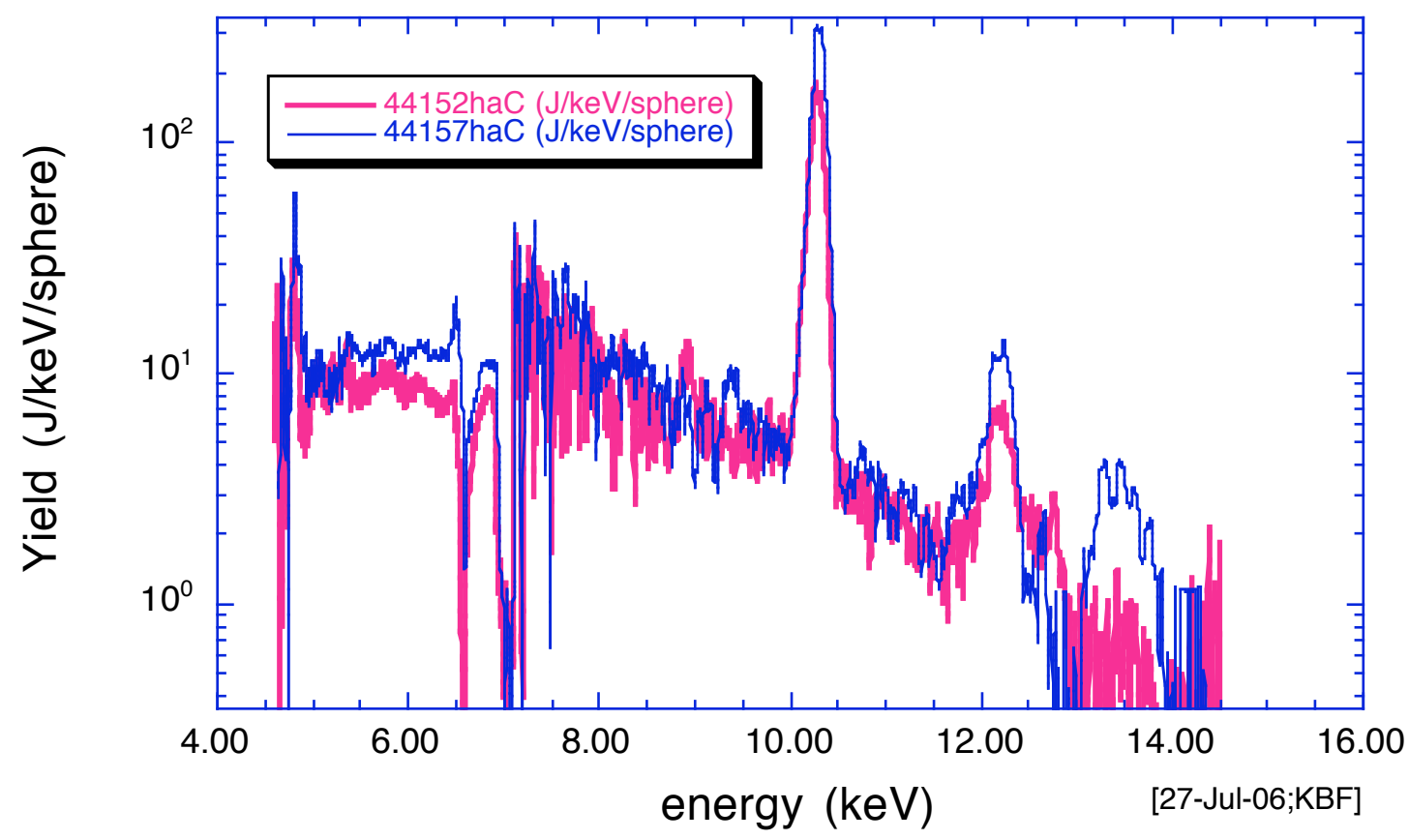

Fig. A.4 - Pre-smoothing data from the PET-crystal channel in HENWAY for OMEGA II.

2. What is the spectral resolution of DANTE? I.e., what is the resolution of the lower energy portion of the spectrum?

UPDATE: The answer given below accurately reflects the data reduction for the DANTE data from OMEGA II. However, the text was written before the Dr. Bruno Villette of the CEA was able to finish his (re-)analysis of the DMX data. The state of the art analysis of the DMX yields is given in Appendix D. The differences between the DMX and DANTE yields are tightly clustered around an average value of $\approx 7 \%$. The close agreement between the DMX and DANTE yields gives us good confidence in the DANTE results for OMEGA II.

Mark May has processed the DANTE signals using the LLNL IDL code that has been developed for the OMEGA DANTE (and is being improved for use as the standard data reduction tool for the NIF DANTE). For the signals from OMEGA II, Mark has used a $50 \mathrm{eV}$ convolution to create the spectral fluxes that were sent around. From the plot that compares the DANTE to the DMX signals in the text above (Fig. 7), it is clear that the French have a much-lower-resolution model in their DMX unfold software (no longer true, Fig. 7 now has the high-resolution model used by Bruno Villette, the two measurements are now in close agreement). A table of Mark's comparison of the DANTE and DMX signals for our 02/24/2006 shots is given in Table A.1 below: 


\begin{tabular}{|c|c|c|c|c|c|}
\hline Shot no. & $\begin{array}{c}\text { DANTE } \\
(\mathrm{J} / \text { ster })\end{array}$ & $\begin{array}{c}\text { DMX } \\
(\mathrm{J} / \text { ster })\end{array}$ & $\%$ difference & $\begin{array}{c}\text { DMX } \\
(\mathrm{J} / \text { ster }) \\
\end{array}$ & $\%$ difference \\
\hline 42749 & 755.621 & 505.134 & 49.6 & 734 & $3 \%$ \\
\hline 42750 & 796.990 & 541.290 & 47.2 & 720 & $11 \%$ \\
\hline 42751 & 591.835 & 486.363 & 21.7 & 671 & $12 \%$ \\
\hline 42752 & 867.554 & 598.514 & 45.0 & 821 & $6 \%$ \\
\hline 42753 & -- & -- & -- & & \\
\hline 42754 & 675.720 & 494.293 & 36.7 & 683 & $1 \%$ \\
\hline 42755 & 268.000 & 197.097 & 36.0 & 242 & $11 \%$ \\
\hline 44152 & 573.889 & & & & \\
\hline 44153 & 662.822 & & & & \\
\hline 44154 & 859.773 & & & & \\
\hline 44156 & 851.373 & & & & \\
\hline 44157 & 455.807 & & & & \\
\hline 44158 & 655.395 & & & & \\
\hline
\end{tabular}

Table A.I - DANTE and DMX yields from Ge-doped aerogel plasma radiation sources. Reported yields are integrated between 30 and $3000 \mathrm{eV}$. The percentage difference is relative to the DMX signal. DMX YIELDS IN THE LAST TWO COLUMNS ARE THE NEW ONES FROM 10/06, see Appendix D.

The most interesting thing to note from Table A.I is that for shots 44152 and 44157 , our pre-shot design signals were taken from the DMX measurements for 42751, which (overall) agrees pretty well with the DANTE result for 42751. For the other OMEGA II shots (1 ns ones), the pre-shot design was done with the DMX signal for 42750; the DANTE signal is quite a bit higher than DMX for 42750, but the measured signals from the OMEGA II shots are pretty close to the DANTE signal for 42750 (except for 44153 and 44158, which had the strange temporal waveforms).

As stated in Section II of the report above, we believe the error on the DANTE signals may be biased towards an overestimate, by as much as $35-40 \%$ of the stated yields.

3. The LLNL PCD H11 -- is this a standard 0.5mm thick diamond PCD? Did you apply a bias correction to these signals? What scope was it recorded on? (i.e., 6804?)

The "standard" PCDs used by LLNL are $1 \times 1 \times 1 \mathrm{~mm}$ cubes. Yes, the signal for the LLNL H11 PCD is bias corrected. It was recorded on the same scopes as the DANTE channels, SDC5000s. Chuck Sorce and Steve MacLaren have provided the answers to these questions, which are now in the text of the report.

4. What is the temporal resolution for the DANTE signals? (i.e., what was it recorded on?) Is it cable compensated? 
Since the H11 PCD is recorded on the DANTE scopes, SDC5000s. Again, Chuck Sorce has provided this information, which is in the report.

5. The PCD $66 k 10$ was recorded on a 6804. Using this signal for the comparison should provide the most appropriate temporal resolution. Let me know if you don't have this and I can forward it.

Comparison of the p126k10, the p66k10 and the H11 PCD are now below in Table A.III. In Section III of the text of the report, we recommend using the p66k10 waveforms for any applications that require them.

6. I don't know where your PCD numbers in table II came from. These do not agree with the numbers that I sent out on 7/21/06 (either for what I labeled as Soft filter, or 5 mil kapton). I do have softer filters that could be compared with DANTE 1-3 keV if we subtract off the higher energy portions.

The numbers I have for the SNL PCD yield estimates come from the spreadsheet you sent on 7/21/06 (OMEGAII_fluences706.xls) from the "soft filter" column in the block labeled "All yields". You have the numbers labeled as (J), which I assumed was $\mathrm{J} /$ sphere. To convert to $\mathrm{J} / \mathrm{sr}$, I divided by $4 \pi$, which gives the numbers I have in Table II of the 8/28/06 report (presented again in Table A.II):

\begin{tabular}{|c|c|c|}
\hline Shot & $\mathrm{Y}(\mathrm{J})[\mathrm{CAC}]$ & $\mathrm{Y}(\mathrm{J} / \mathrm{sr})[\mathrm{KBF}]$ \\
\hline 1 & 3700 & 294.44 \\
\hline 2 & 3920 & 311.94 \\
\hline 3 & 3655 & 290.86 \\
\hline 4 & 3906 & 310.83 \\
\hline 5 & 3300 & 262.61 \\
\hline 6 & 4047 & 322.05 \\
\hline
\end{tabular}

Table A.II - Table of the "soft X-ray yields" found by the SNL-PCDs as sent on $7 / 21 / 06$; these are very preliminary numbers and will change with further analysis.

7. I would suggest not referring to them as "1-ns" and "3-ns" pulses. The FWHM of the "1-ns" is near $1 \mathrm{~ns}$, but the FWHM of the "3-ns" is approx 1.6 ns for most cases. While there may be energy over 3 ns if you go down to the foot, this just seems like inconsistent terminology to me.

Good point. I'll (probably) change it to something more appropriate in future documents.

CAC: Other notes on the PCDs: My estimates of FWHM (from P66k10, the one on the 6804) are: $1.5,1.85,1.15,1.2,1.6$, and $1.3 \mathrm{~ns}$ (shots 1-6). These are taken after the PCDs have been compensated and bias corrected. These are somewhat different from what you have in table 3. 
Below, Table A.III summarizes our analysis of the PCD FWHM for the harder X-ray signals. I have listed again the laser power and H11 PCD FWHM. These values were found by fitting the waveforms with a Gaussian envelope using KaleidaGraph ${ }^{\mathrm{TM}}$. The H11 PCD data were cable compensated and bias corrected. I also have listed the FWHM for the X-ray waveforms as measured with your p66k10 (recorded on a TDS 6804B) and your p126k10 (recorded on a TDS 694C). I have taken you data, and shifted it so that the peak of the signal is approximately at zero ns. Then, I fit the data between -3 and $10 \mathrm{~ns}$ for p66k10, and between -2 and $10 \mathrm{~ns}$ for p126k10 with Gaussians. The results for the FWHM are listed below. Also listed are your values, which you derived using XDAMP. I think we can safely say that the Gaussian approximation is not bad except for the case of the second and sixth shots, which are identified above (Section III) as having very strange waveforms, with extended early- and late-time features. Overall, my Gaussian fits and your values are closer than $100 \mathrm{ps}$. The values from the H11 PCD and the p66k10 PCD (based on your analysis) are overall quite different, everywhere more than $200 \mathrm{ps}$, except for the third and fifth shots. These observations are also true with respect to your analysis of the p126k10 signals. This points to a difference in the devices. This is something we can pursue.

\begin{tabular}{|c|c|c|c|c|c|c|}
\hline Shot no. & $\begin{array}{c}\text { Laser } \\
\text { FWHM } \\
\text { (ns) }\end{array}$ & $\begin{array}{c}\text { H11 PCD } \\
\text { FWHM } \\
(\mathrm{ns})\end{array}$ & $\begin{array}{c}\mathrm{p} 126 \mathrm{k} 10 \\
{[\mathrm{KBF}]} \\
\text { FWHM } \\
(\mathrm{ns})\end{array}$ & $\begin{array}{c}\mathrm{p} 126 \mathrm{k} 10 \\
\text { [CAC] } \\
\text { FWHM } \\
\text { (ns) }\end{array}$ & $\begin{array}{c}\text { p66k10 } \\
{[\mathrm{KBF}]} \\
\text { FWHM } \\
(\mathrm{ns})\end{array}$ & $\begin{array}{c}\text { p66k10 } \\
\text { [CAC] } \\
\text { FWHM } \\
\text { (ns) }\end{array}$ \\
\hline 44152 & 1.68 & 1.97 & 1.44 & 1.45 & 1.51 & 1.5 \\
\hline 44153 & 1.00 & 1.54 & 1.62 & 1.75 & 1.70 & 1.85 \\
\hline 44154 & 1.00 & 1.02 & 1.08 & 1.10 & 1.19 & 1.15 \\
\hline 44156 & 1.00 & 1.02 & 1.11 & 1.15 & 1.21 & 1.2 \\
\hline 44157 & 1.71 & 1.64 & 1.52 & 1.45 & 1.63 & 1.6 \\
\hline 44158 & 1.00 & 1.74 & 1.52 & 1.25 & 1.57 & 1.3 \\
\hline
\end{tabular}

Table A.III - Comparison of the FWHM for X-ray waveforms as fitted by KBF and CAC using the SNL PCD p126k10 (on a TDS 694C) and the SNL PCD P66k10 (on a TDS 6804). Also listed is KBF's fit to LLNL's H11 PCD.

CAC: I will likely be putting together a similar report for our internal program use, and one question I have for that report is: Did we do this target during any of the Omega I shots? (i.e., all of the Omega II targets were the same, but is there a direct comparison for me from Omega I?)

The answer is no. All the OMEGA I shots were either density 4.8 or $6.5 \mathrm{mg} / \mathrm{cm}^{3}$ aerogels in Be cylinders with $1.5 \mathrm{~mm}$ ID $\times 1.2 \mathrm{~mm}$ length. The OMEGA II loads were all $3.7 \mathrm{mg} / \mathrm{cm}^{3}$ aerogels in $2.0 \mathrm{~mm}$ ID $\times 2.2 \mathrm{~mm}$ length cylinders. Further, the focusing of the beams in OMEGA I was much tighter, which led to intensities on the order of $6 \times$ $10^{16} \mathrm{~W} / \mathrm{cm}^{2}$, while in OMEGA II the focusing was broad for the 1-ns pulses (giving $\mathrm{I}_{\text {las }} \approx$ $2 \times 10^{15} \mathrm{~W} / \mathrm{cm}^{2}$ ) or moderate for the 3 -ns pulses (which, with the longer duration, still had intensities on the order of $3 \times 10^{15} \mathrm{~W} / \mathrm{cm}^{2}$ ). 
There were some commonalities between the OMEGA I loads and the 02/24/06 targets, and the final design for the OMEGA II loads were exactly the same as the 02/24/06 targets for shots 42750 and 42751; see Appendix B. 


\section{Appendix B}

Appendix B attempts to give the reader some feel for the variability in the X-ray yields from nominally identical shots on OMEGA of laser-driven Ge-doped aerogel targets. The only case where there are identical configurations are in OMEGA I, where targets with the same size, density, laser focusing (laser intensity) and laser pulse shape were shot $(39152,54,56$, and 57) and $(39153,58)$, and between the $02 / 24 / 06$ shots and the OMEGA II shots $(42750,44153,54,56,58)$ and $(42751,44152$, and 57). Plots that summarize target performance as functions of density, target size and laser intensity are also presented.

Figure B.1 shows the HENWAY-derived yields from OMEGA I in the $5-15$ $\mathrm{keV}$ band, as found from the (re-)analysis of the data done in mid-July, 2006. All HENWAY data from OMEGA I, 10/18/05, 02/24/06 and OMEGA II were re-analyzed with a consistent set of cuts through filter packs on the film images, consistent sets of background cuts on the unexposed film, and a consistent algorithm for fitting a smooth continuum to the reduced data. The data from OMEGA I have targets of two densities $\left(4.8\right.$ and $\left.6.5 \mathrm{mg} / \mathrm{cm}^{3}\right)$, held in 'small' cylinders of $1.5 \mathrm{~mm} \mathrm{ID} \times 1.2 \mathrm{~mm}$ length, shot with very tight laser focusing. The second and sixth shots were both at the higher density, with the target sizes being identical, and the laser power in 39158 slightly broadened from the laser power in 39153 (1.2 ns vs. $1.0 \mathrm{~ns})$. The yields from HENWAY are very similar. The other shots $(39152,39154,39156,39157)$ were all at the lower density, with shot 39157 also having the slightly broadened laser power. The yield of the third shot, 39154, is significantly higher than the other three shots in this subset of data, the reason for this, other than the fact that only in this shot do the heated lobes of plasma from each end of the cylinder merge during the course of the shot, is not known. (See in particular Ref. [b.1] and also Ref. [b.2].) The remaining three shots in this set (39152, 39156, 39157) are relatively close in X-ray yield; it should be noted that three of 40 laser beams were offline for shots 39152 and 39153, which means that those shots had nearly $1.5 \mathrm{~kJ}$ less laser energy than the others. In terms of reproducibility, from the HENWAY data for OMEGA I, for the $4.8 \mathrm{mg} / \mathrm{cm}^{3}$ targets, one can expect $159.3 \pm 76.8 \mathrm{~J} / \mathrm{sphere}$ in the $5-$ $15 \mathrm{keV}$ band, and for the $6.5 \mathrm{mg} / \mathrm{cm}^{3}$ targets, $77.9+7.3 \mathrm{~J} / \mathrm{sphere}$ for the same band.

The data from the SNL PCDs, after cable compensation of the waveforms, and correction of the signals for the PCD biasing are also shown. The PCD yields reflect Xray yields from all energies above $4 \mathrm{keV}$. These numbers have been considered final, but as the OMEGA I HENWAY spectra were re-analyzed in July 2006 in order to have a smooth continuum, the SNL PCD yields may be changed as the PCD signals are reconsidered given the new spectral energy distributions to be used in the unfolds. For the given data in Fig. B.1, the agreement between the SNL PCDs and the HENWAY data is quite close, and well within the error bars for both diagnostics. The HENWAY signals must have the fitted continuum extrapolated to $4 \mathrm{keV}$ in order to have a truly accurate comparison.

As an aside, it is mentioned that the original idea in OMEGA I was to use the 1.5 $\times 1.2 \mathrm{~mm}$ targets, thinking the smaller target volume would get hotter for the same drive energy than the larger targets described in [b.2], thus maximizing Ge K-shell emission. It seems, based on comparisons with $02 / 24 / 06$ data, that this is not the case. 


\section{July06_HENWAY_SNL_PCD_comp}

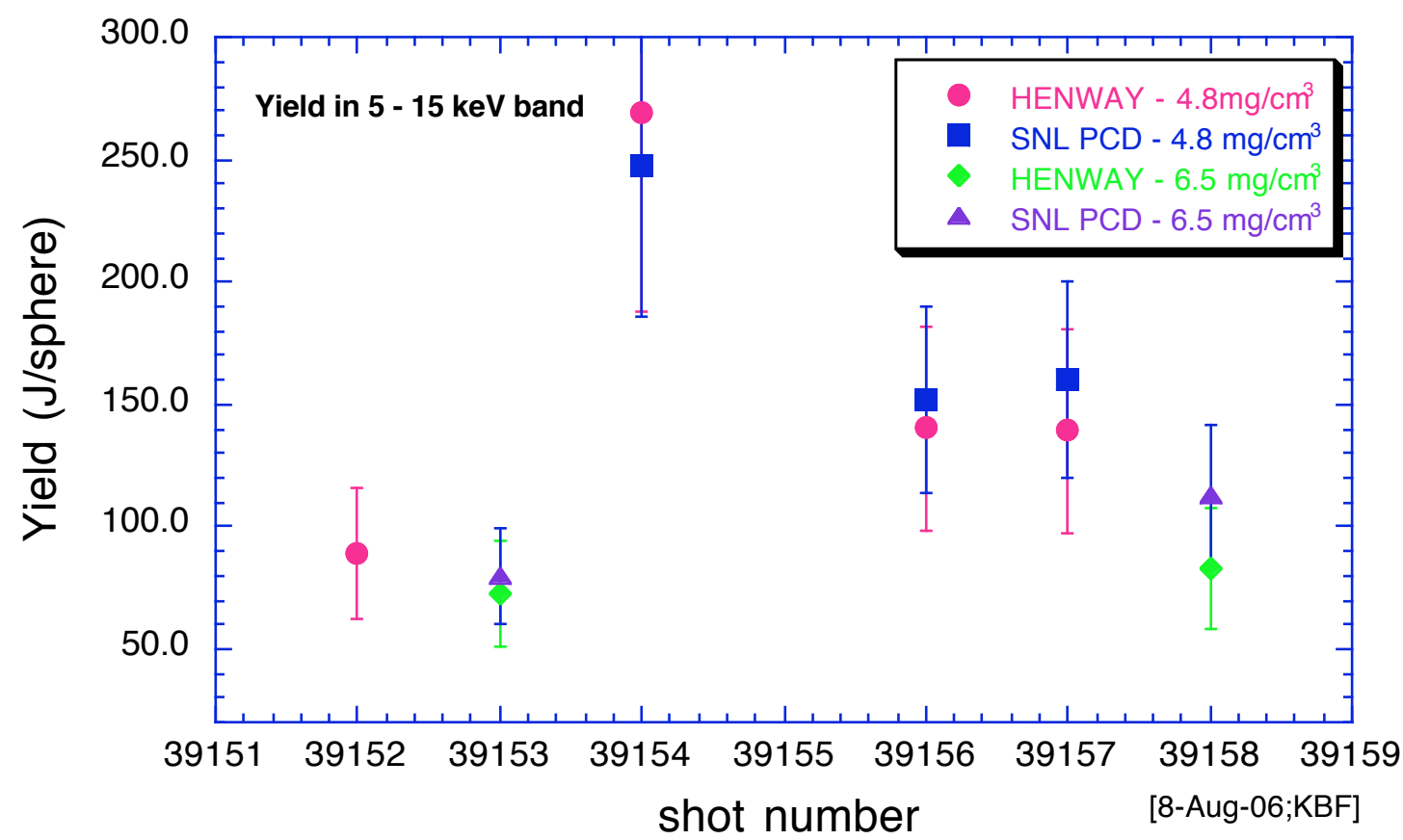

Fig. B.1 -OMEGA I X-ray yield numbers for the $5-15 \mathrm{keV}$ band from HENWAY (channel A), and from the high-energy signals from the SNL PCDs, which reflect yields generally above $4 \mathrm{keV}$. The HENWAY numbers should be extrapolated down to 4.0 $\mathrm{keV}$, the SNL PCD numbers could change if the revised OMEGA I HENWAY spectral data are used in reconsidering the PCD unfold. The agreement between the two diagnostics is excellent. Overall, the consistency between identical targets on nominally identical shots is very good for the two high-density targets, and good, but with more variation for the four lower-density targets.

Figure B.2 shows the data from the 02/24/06 Ge-doped-aerogel target shots. There were no configurations repeated during the day on those shots. The first six shots of the day were in larger targets than used for OMEGA I (2.0 mm ID $\times 2.2 \mathrm{~mm}$ length). The last shot of the day was in one of the $1.5 \times 1.2 \mathrm{~mm}$ targets from OMEGA I, but the cylinder was filled with a $3.6 \mathrm{mg} / \mathrm{cm}^{3}$ aerogel, as opposed to the 4.8 or $6.5 \mathrm{mg} / \mathrm{cm}^{3}$ gels in OMEGA I. The first two shots of the day had the same 1 ns laser-power pulse (pulse shape SG1018) delivered to both faces of the cylindrical target, but with the laser beam focusing changed by a large amount for the cone 2 and 3 beams in particular; both shots, 42749 and 50 were defocused compared to the beam focusing used in OMEGA I. The defocused shot 42750 performed much better than the so-called NWET focusing shot 42749. The next two shots had '3-ns' laser-power pulse with NWET and a broadly defocused laser configuration. In this case, the NWET-focusing shot, 42751, performed somewhat better than 42752. Shot 42753, the fifth shot, was a 6-ns long laser pulse, and gave a barely measurable hard-X-ray yield (although its soft-X-ray output was quite good). The sixth shot, 42754, had one of the $2.0 \times 2.2 \mathrm{~mm}$ cylinders, filled with 4.6 $\mathrm{mg} / \mathrm{cm}^{3}$ aerogel (the previous five shots were all on targets filled with $3.5 \mathrm{mg} / \mathrm{cm}^{3}$ 
aerogel), and performed quite well. In fact, this density, which is close to that used consistently in OMEGA I, performed better than all $4.8 \mathrm{mg} / \mathrm{cm}^{3}$ OMEGA I targets except the exceptional 39154. (NOTE: the spectral ranges in Figs. B.1 and B.2 are different, the comparison just mentioned is for the range 5-15 keV.) This suggests that the larger-size target is preferable, particularly if the phenomenon(a) that made 39154 so bright can be understood, reproduced, and made to scale to the larger-size target. The last target of the day, 42755, was a $1.5 \times 1.2 \mathrm{~mm}$ cylinder with the $3.5 \mathrm{mg} / \mathrm{cm}^{3}$ aerogel, and it performed below all OMEGA I targets.

\section{July06_HENWAY_DMX_comp}

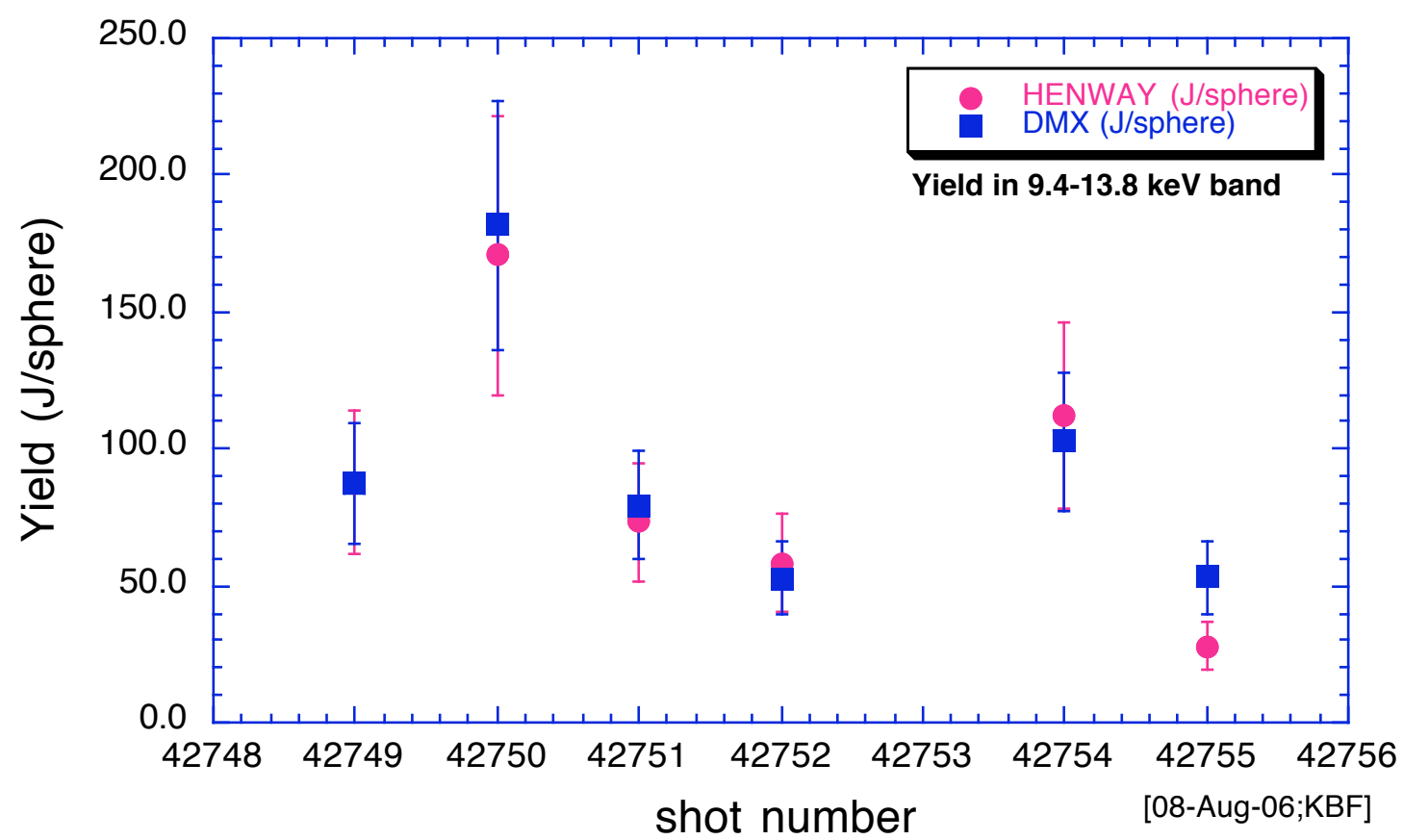

Fig. B.2 - OMEGA 02/24/06 X-ray yield numbers for the Ge K-shell band from HENWAY (channel A), and from the high-energy signals from the CEA's DMX, which puts flux into a single effective line and continuum. The agreement between the two diagnostics is excellent. There are no identical targets or nominally identical shots from this series.

Figure B. 2 also shows the yields for the 02/24/06 shots as measured by the CEA's DMX diode array. The diagnostic has been described in Ref. [b.3]. The agreement for all targets and all laser configurations is excellent, with only the small cylinder, 42755 not having overlap between the two diagnostics' error bars. The $4.5-15 \mathrm{keV}$ yields from HENWAY and DMX for 02/24/06 are plotted in Fig. B.3. The agreement is far less satisfactory when one looks at the range from $4.5-15 \mathrm{keV}$; the DMX signals are consistently about a factor of two larger. One can compare the yields from 42754 and 42755 to the yields for $(39152,54,56,57)$ in Fig. B.1. 


\section{Jan07_HENWAY_DMX_comp}

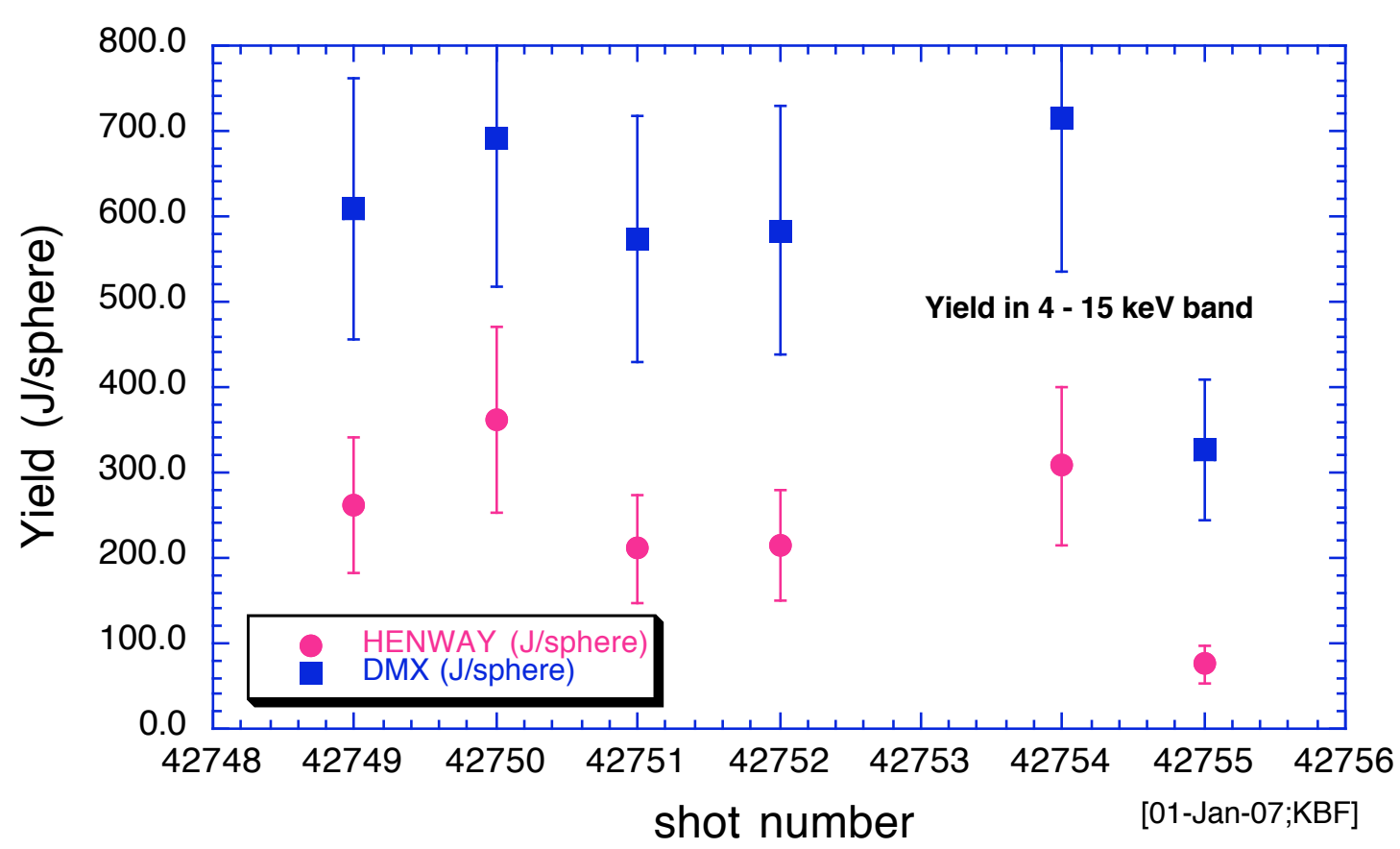

Fig. B.3 - OMEGA 02/24/06 X-ray yields in the 4.5 - $15 \mathrm{keV}$ band from HENWAY (channel A), and from the high-energy signals from the CEA's DMX, which puts flux into a single, effective line and continuum. There are no identical targets or nominally identical shots from this series; shot 42754 is shot at nearly the same density as some OMEGA I targets, but with a larger cylinder. Shot 42755 is shot in a cylinder the size of those in OMEGA I, but with a density that is about $25 \%$ lower.

Figure B.4 summarizes the data in the $4-15 \mathrm{keV}$ band for the shots from OMEGA II. Four of the shots $(44153,54,56$, and 58) are identical with 1 ns laser irradiation, $2.0 \mathrm{~mm}$ ID $\times 2.2 \mathrm{~mm}$ length cylinders, and aerogel densities of $3.7 \mathrm{mg} / \mathrm{cm}^{3}$. The yields from HENWAY and the SNL PCDs follow a very similar trend, with the PCD yields consistently higher than the HENWAY yields. It is interesting to note that both diagnostics show the targets' output increasing as the day progressed. There is no explanation for why this might be, or even an expectation that the trend should exist. It is only for the two 3 ns shots $(44152,57)$ that the signals from the two diagnostics overlap in their error bars. Again, from the point of view of reproducibility, one can expect 239.3 $\pm 80.5 \mathrm{~J} /$ sphere for the $1 \mathrm{~ns}$ shots, and $180.2 \pm 35.7 \mathrm{~J} /$ sphere for the $3 \mathrm{~ns}$ shots. Overall, the consistency between identical targets on nominally identical shots is good for the two 3 ns shots, and not so good for the 1 ns shots, with a seemingly increasing trend with time. 
Jan07_HENWAY_SNL_PCD_comp

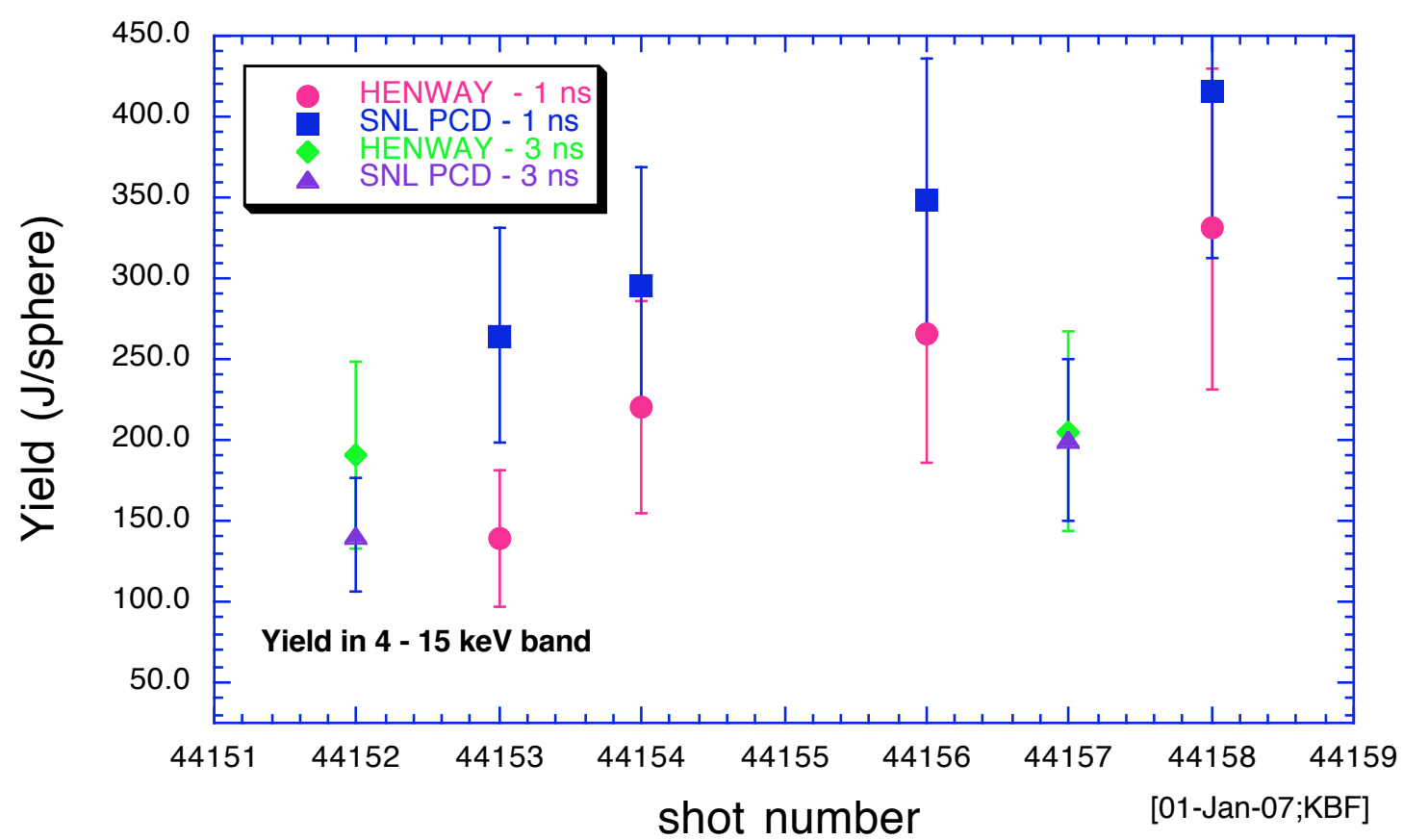

Fig. B.4 - OMEGA II X-ray yield numbers for the $4-15 \mathrm{keV}$ band from HENWAY (channel A), and from the high-energy signals from the SNL PCDs, which reflect yields generally above $4 \mathrm{keV}$. The agreement between the two diagnostics is excellent for the 3 ns shots, but rather poor for the four $1 \mathrm{~ns}$ shots. Overall, the consistency between identical targets on nominally identical shots is good for the two 3 ns shots, and not so good for the 1 ns shots, with a seemingly increasing trend with time.

One last comment about consistency of yields for identical targets can be made: the four $1 \mathrm{~ns}$ shots from OMEGA II were based on the 02/24/06 shot 42750, while the two $3 \mathrm{~ns}$ shots were based on expectations from 42751. The average value for the four $1 \mathrm{~ns}$ shots in the $4-15 \mathrm{keV}, 239.3 \pm 80.5 \mathrm{~J} /$ sphere, is $20 \%$ less than the $4.5-15 \mathrm{keV}$ HENWAY value of $300.6 \mathrm{~J} / \mathrm{sphere}$ for 42750 , which still falls within the $80.5 \mathrm{~J} / \mathrm{sphere}$ statistical uncertainty. The average value for the two $3 \mathrm{~ns}$ shots in the $4-15 \mathrm{keV}$ band, $180.2 \pm 35.7 \mathrm{~J} /$ sphere, is close to the $4.5-15 \mathrm{keV}$ HENWAY value of $163.1 \mathrm{~J} /$ sphere for 42751.

Figure B.5 presents the complete set of yields in the $9-15 \mathrm{keV}$ band for shots with 20 atom-\% Ge-doped aerogel targets at OMEGA as a function of target density. These are the recommended yields, so they reflect averages of HENWAY yields (sometimes from more than one cut on a piece of film) and DMX of scaled SNL PCD yields. The icons in Fig. B.5 have been sorted into categories with common target sizes and/or laser intensities or pulse lengths. There seems to be a modest trend in yield with target density, the averages of yields around the $3.6,4.8$ and $6.5 \mathrm{mg} / \mathrm{cm}^{3}$ densities have been plotted as the black diamonds along with the standard deviation around the average, and fitted with a straight line. The three densities above correspond to 10,15 and $20 \%$ of the critical density for $3 \omega(351 \mathrm{~ns})$ laser light, respectively. One can see why $3.6 \mathrm{mg} / \mathrm{cm}^{3}$ 
was chosen as the target density for OMEGA II. A sense of the variability in X-ray yield for similar targets and laser configurations can be gleaned from the spread in the icons for a given set of conditions in Fig. B.5 (as well as for identical targets and similar laser conditions in OMEGA I and II as shown in the plots in Figs. B.1 and B.4).

\section{ge_v_density_070101}

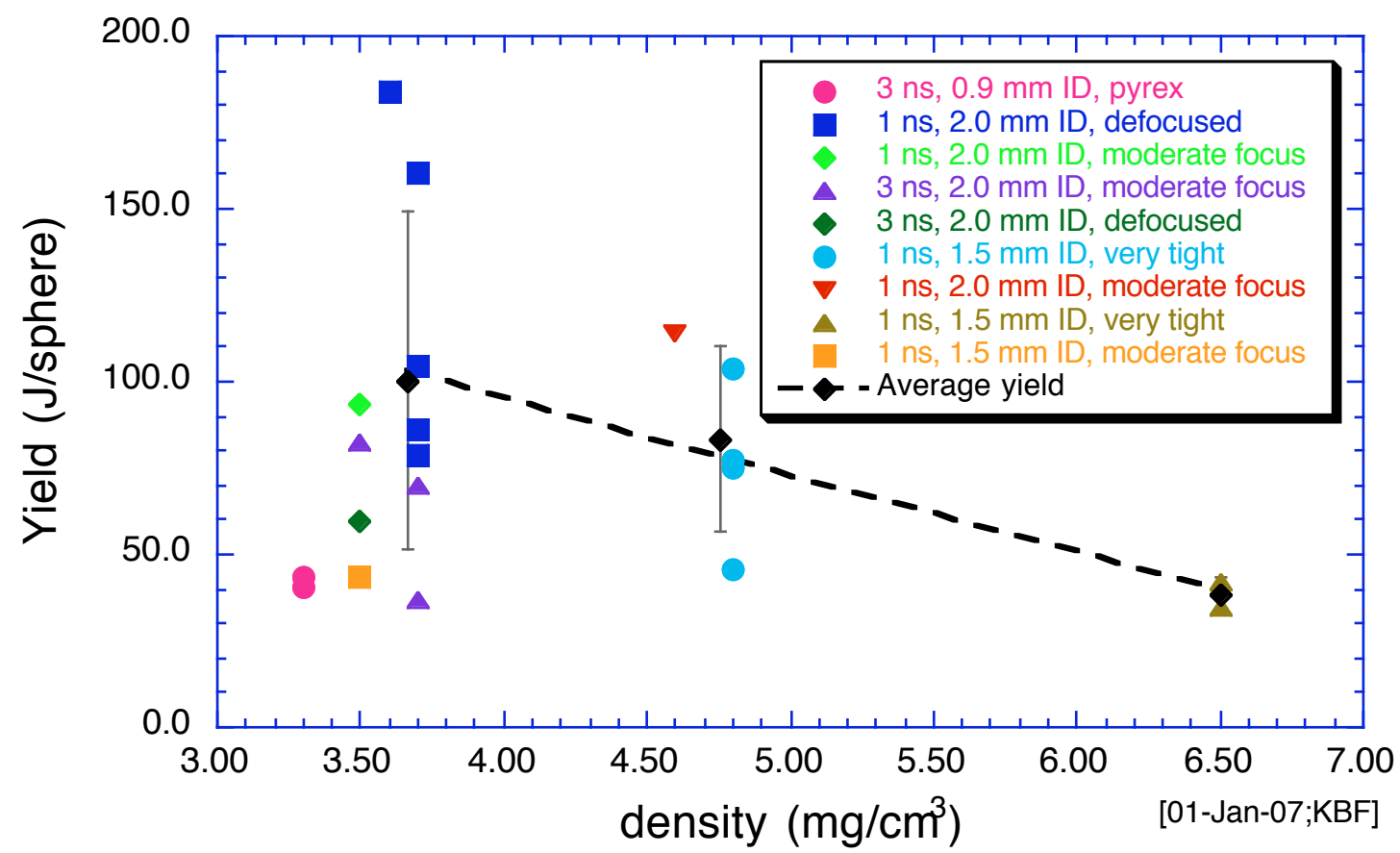

Fig. B.5 - Ge-doped aerogel yield data in the $9-15 \mathrm{keV}$ band for all shots at OMEGA in the 2005 and 2006 campaigns. Variability in expected yields for a given target and laser condition can be inferred from the scatter in any set of icons.

Figure B.6 presents the complete set of yields in the $9-15 \mathrm{keV}$ band for shots with 20 atom-\% Ge-doped aerogel targets at OMEGA as a function of target size (inner diameter of the LEH, actually - the targets from OMEGA I had different lengths than those in OMEGA II or on 02/24/06). These are the recommended yields, so they reflect averages of HENWAY yields (sometimes from more than one cut on a piece of film) and DMX of scaled SNL PCD yields. The icons in Fig. B.6 have been sorted into categories with common target densities and/or laser intensities or pulse lengths. There seems to be a good trend in yield with target size, the averages of yields around $0.9,1.5$ and $2.0 \mathrm{~mm}$ inner diameter have been plotted as the black diamonds along with the standard deviation around the average, and fitted with a straight line. One can see why $2.0 \mathrm{~mm} \times 2.2 \mathrm{~mm}$ length was chosen as the target size for OMEGA II. It would seem that from the increasing trend in Fig. B.6, the optimal target size has not yet been found, and that we may be able to drive a bigger target to still higher yields. This is being assessed with radiation-hydrodynamic modeling. A sense of the variability in X-ray yield for similar targets and laser configurations can be gleaned from the spread in the icons for a given 
set of conditions in Fig. B.6 (as well as for identical targets and similar laser conditions in OMEGA I and II as shown in the plots in Figs. B.1 and B.4).

ge_v_size_070101

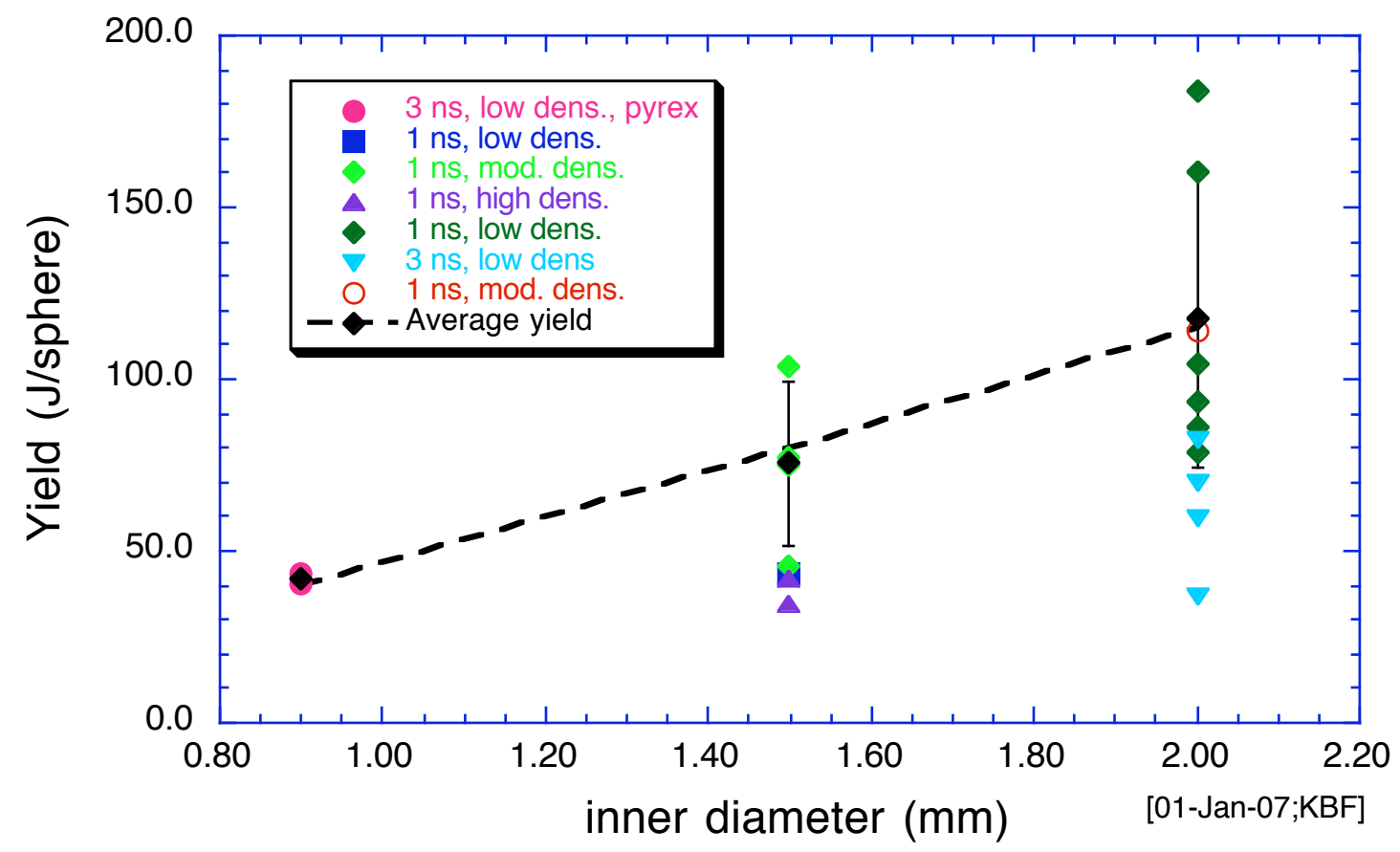

Fig. B.6 - Ge-doped aerogel yield data in the $9-15 \mathrm{keV}$ band for all shots at OMEGA in the 2005 and 2006 campaigns. Variability in expected yields for a given target and laser condition can be inferred from the scatter in any set of icons.

Figure B.7 presents the complete set of yields in the $9-15 \mathrm{keV}$ band for shots with 20 atom- $\%$ Ge-doped aerogel targets at OMEGA as a function of laser intensity onto each cylindrical face of the target. These are the recommended yields, so they reflect averages of HENWAY yields (sometimes from more than one cut on a piece of film) and DMX of scaled SNL PCD yields. The icons in Fig. B.7 have been sorted into categories with common target densities and/or target sizes and laser-pulse lengths. The averages of yields around two intensities have been plotted as the black diamonds along with the standard deviation around the average, and fitted with a power-law curve. There seems to be only a weak trend in yield with laser intensity, however, the two highest-performing targets of all these shots were for defocused conditions with $\mathrm{I}_{\text {las }} \approx 2 \times 10^{15} \mathrm{~W} / \mathrm{cm}^{2}$. However, the one super-performing shot from OMEGA I was at $\mathrm{I}_{\text {las }} \approx 6 \times 10^{16} \mathrm{~W} / \mathrm{cm}^{2}$. One can see why the defocused condition was chosen as the laser focusing to be used for 1 ns shots in OMEGA II (similarly the NWET focusing for the 3 ns shots). A sense of the variability in X-ray yield for similar targets and laser configurations can be gleaned from the spread in the icons for a given set of conditions in Fig. B.7 (as well as for identical targets and similar laser conditions in OMEGA I and II as shown in the plots in Figs. B.1 and B.4). 
ge_v_intensity_070101

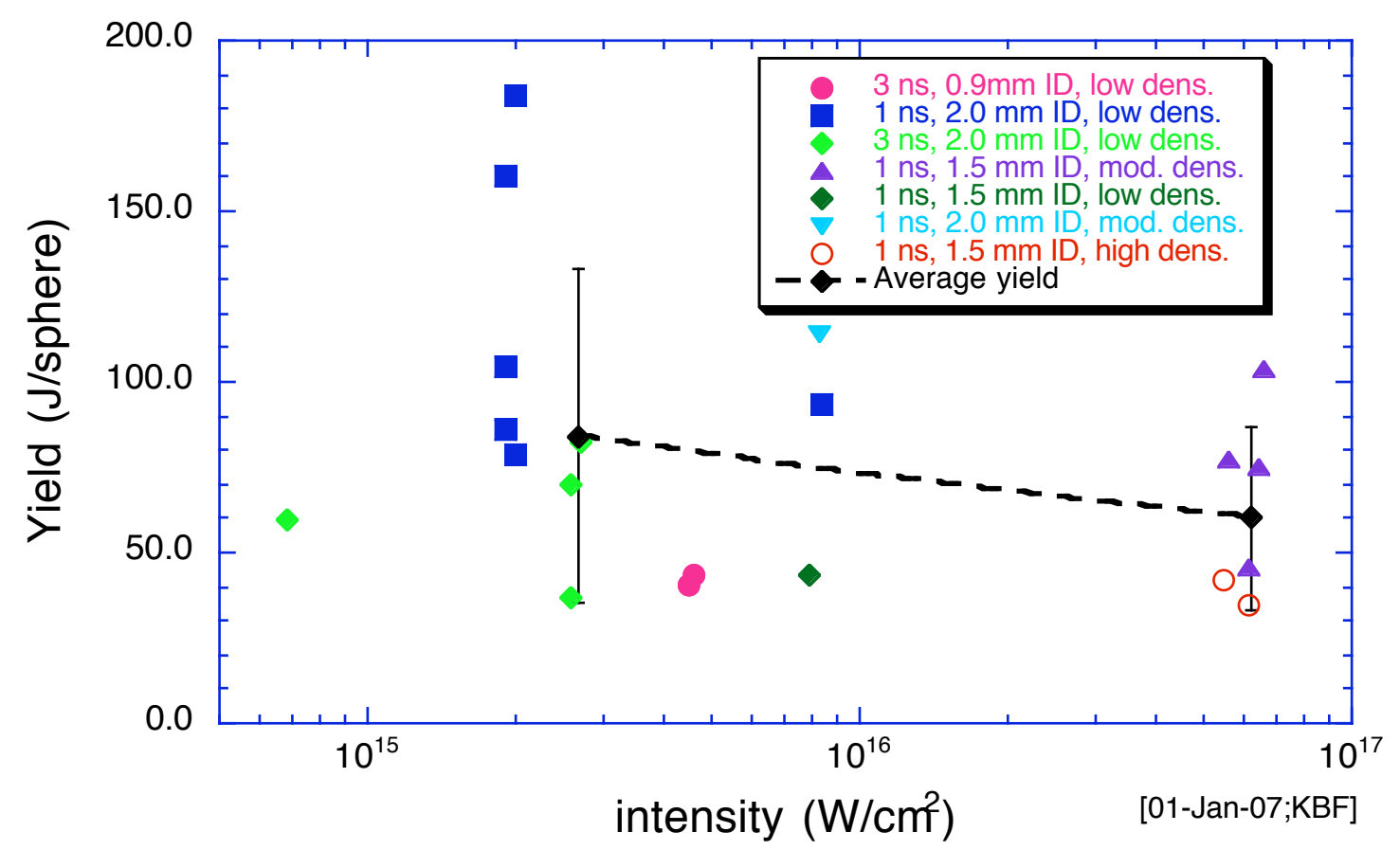

Fig. B.7 - Ge-doped aerogel yield data in the $9-15 \mathrm{keV}$ band for all shots at OMEGA in the 2005 and 2006 campaigns. Variability in expected yields for a given target and laser condition can be inferred from the scatter in any set of icons.

This concludes comments on source reproducibility and expected yields from Gedoped aerogels used as laser-driven-plasma-radiation sources at OMEGA.

\section{Reference:}

[b.1] C. Constantin, C. A. Back, K. B. Fournier, et al., Phys. Plasmas 12, 063104 (2005).

[b.2] K. B. Fournier, C. Constantin, J. F. Poco, et al., Phys. Rev. Lett. 92, 165005 (2004). [b.3] J. L. Bourgade, B. Villette, J. L. Bocher et al., Rev. Sci. Instrum. 72, 1173 (2001). 


\section{Appendix C}

Shown below are the scans of the film detectors used in the HENWAY spectrometer for the OMEGA I, II and the 02/24/06 test days. Features are identified in the images.

\section{OMEGA I:}

Shot 39152, without the Fe foil on channel A, and with good data on Channel B (and redundant signal on channel $\mathrm{C}$ ).

\section{Henway Omega 39152}

A

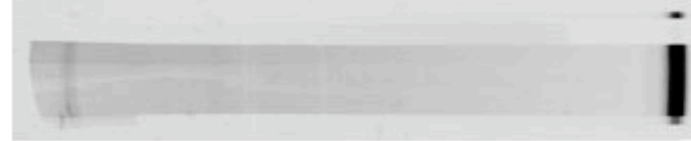

B

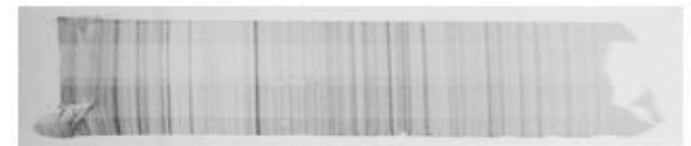

C

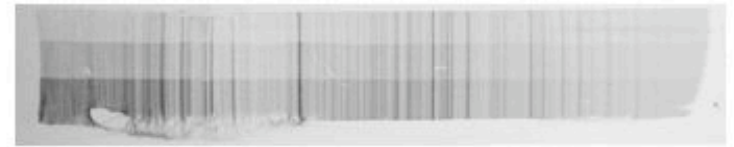

Q-PET

Q-KAP

Q-KAP 
Shot 39156, with the Fe foil on channel A, and with good data on Channel B (and redundant signal on channel C).

\section{Henway Omega 39156}

A

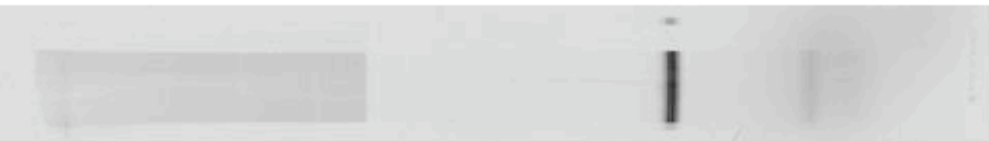

B

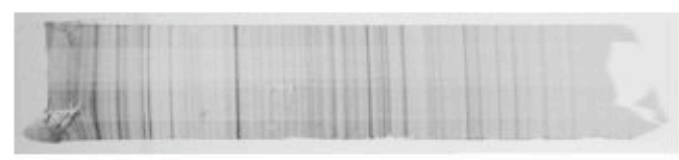

C

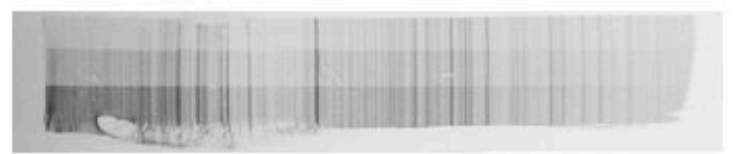

D
Q-PET

\author{
Q-KAP
}

Q-KAP 


\section{2/24/2006:}

Shot 42750, with the Fe foil on channel A (and redundant data on channel C), and with poor (unusable?) data on Channel B. Channel D could provide low-energy spectra.

\section{Henway Omega 42750}

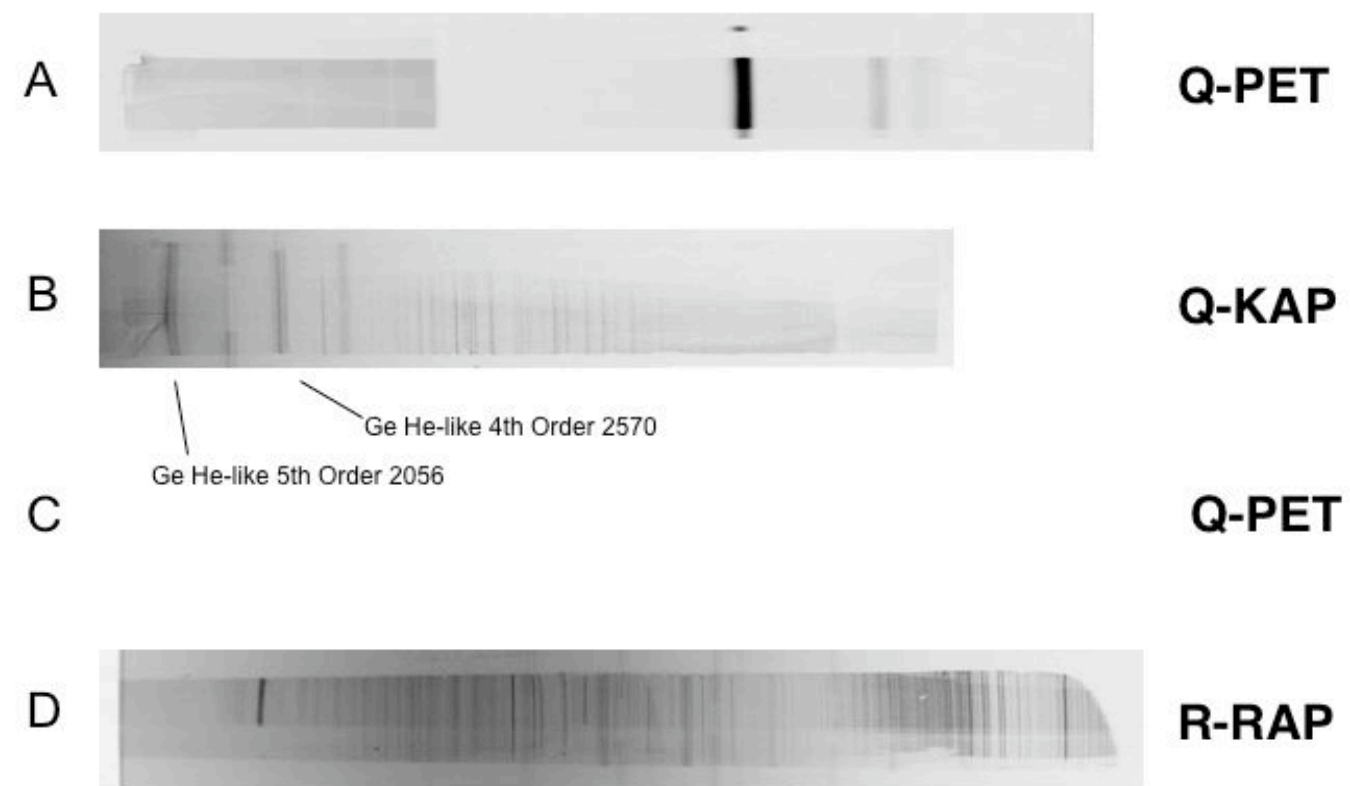


Shot 42751, with the Fe foil on channel A (and redundant data on channel C), and with poor (unusable?) data on Channel B. Channel D could provide low-energy spectra.

\section{Henway Omega 42751}

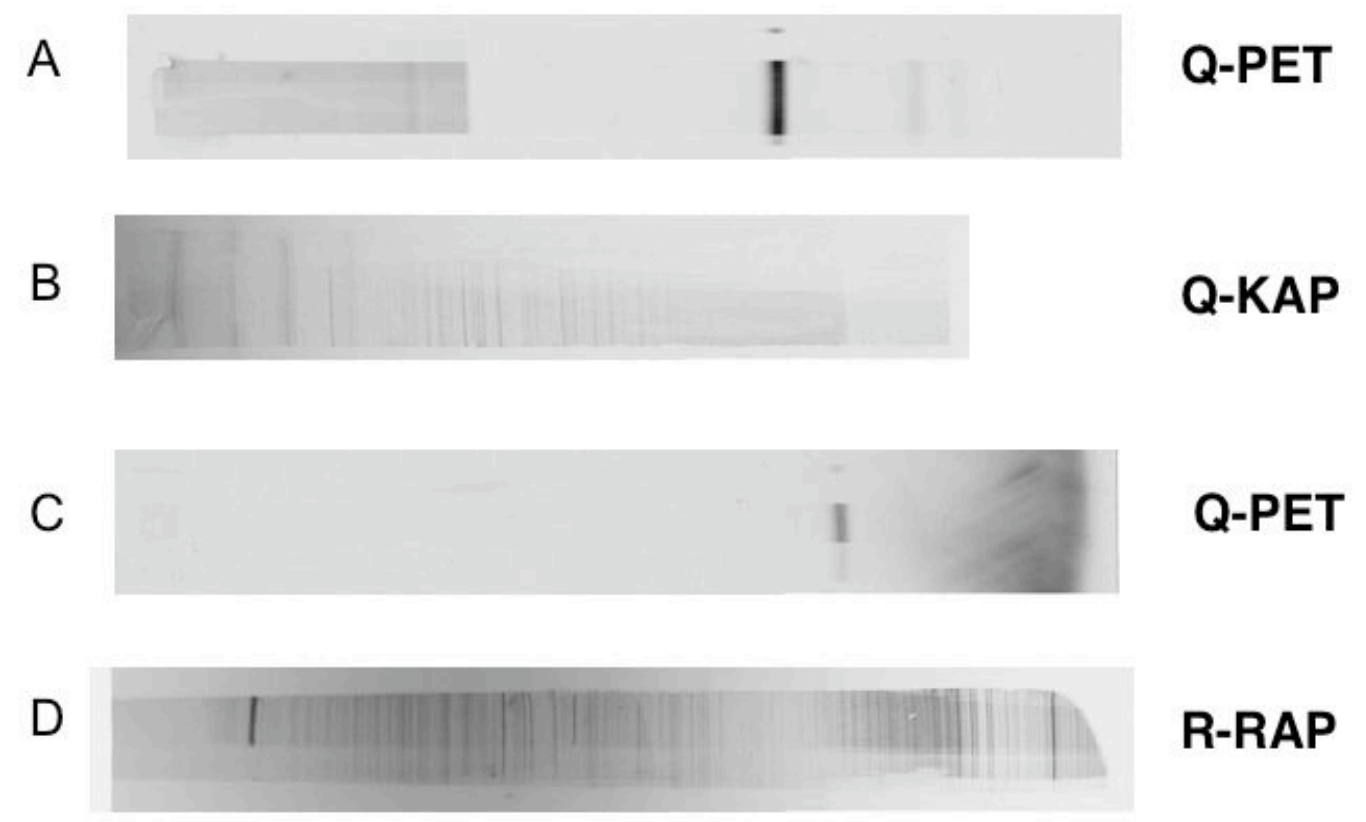




\section{OMEGA II:}

Shot 44156, with the Fe and Ti foils on channel A, and with poor (unusable?) data on Channel B. Channel D could provide low-energy spectra.

\section{Henway Omega 44156}
A
C
Q-PET
D

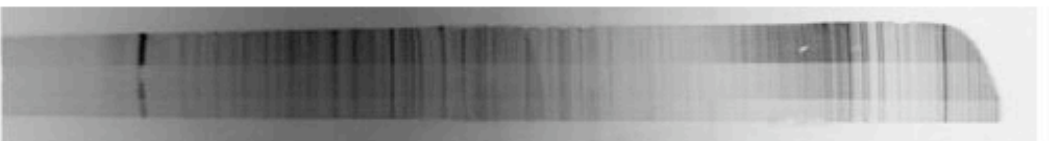
R-RAP 
Shot 44157, with the Fe and Ti foils on channel A, and with poor (unusable?) data on Channel B. Channel D could provide low-energy spectra.

\title{
Henway Omega 44157
}

A

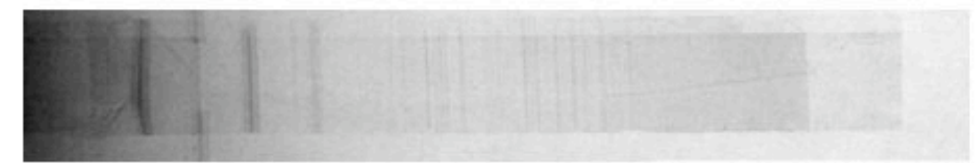

\author{
Q-KAP
}

C

Q-PET

D

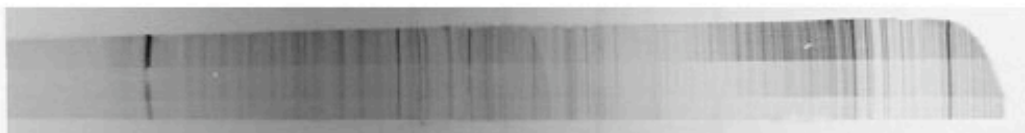

R-RAP 


\section{Appendix D}

The following is an unedited explanation written by Dr. Bruno Villette of the French Commissariat à l'Energie Atomique (CEA) laboratory at Bruyères le Châtel to K. Fournier. Dr. Villette's text explains how he evolved the data reduction of the French DMX diagnostic at the OMEGA laser facility. The final results that came from fitting the DMX signals to a more highly resolved model spectrum now closely match both HENWAY yields in the $9-15 \mathrm{keV}$ spectral range, and the DANTE results in the spectral range below $3 \mathrm{keV}$. Included with Dr. Villette's permission.

\section{Shot 42751}

$1^{\text {st }}$ method of analysis (treatment that I've used to analyze DMX data on your shots. These data are the ones I sent you):

This method consists to calculate in a first step an approximate spectrum (spectrum " 0 ") without any assumption on the theoretical spectra. This spectrum is obtained by interpolation from the experimental data of each channel of the diagnostic divided by their average sensitivity. The second step consists by successive corrections (iterative process) of an initial spectrum (spectrum" 0 ") to reproduce the experimental data, by folding the spectrum with the spectral response of each channel. This data reduction process is classic for this type of broad band measurement but doesn't work very well for a spectrum with thin emission lines. For this case, I remove the channels which measure these lines and I apply the iterative treatment. In this way, I obtain a spectrum (spectrum "1") that consists of soft x-ray emission and continuum. In a third step, I add lines with Gaussian shapes, positioned at right energies and run iterative process giving intensities which permit to reproduce the experimental data (spectrum " 2 "). The last step consists to use the spectrum " 2 " as the initial spectrum of an other iterative process.

I've analyzed DMX data on your shots with this method, but I've only take into account Ge K-shell line (10.25 keV) and not the L-shell emission line (1.6 keV) that's explain the big structure around $2 \mathrm{keV}$ and the differences with Dante. The figures below show the results after the different steps of this treatment. 


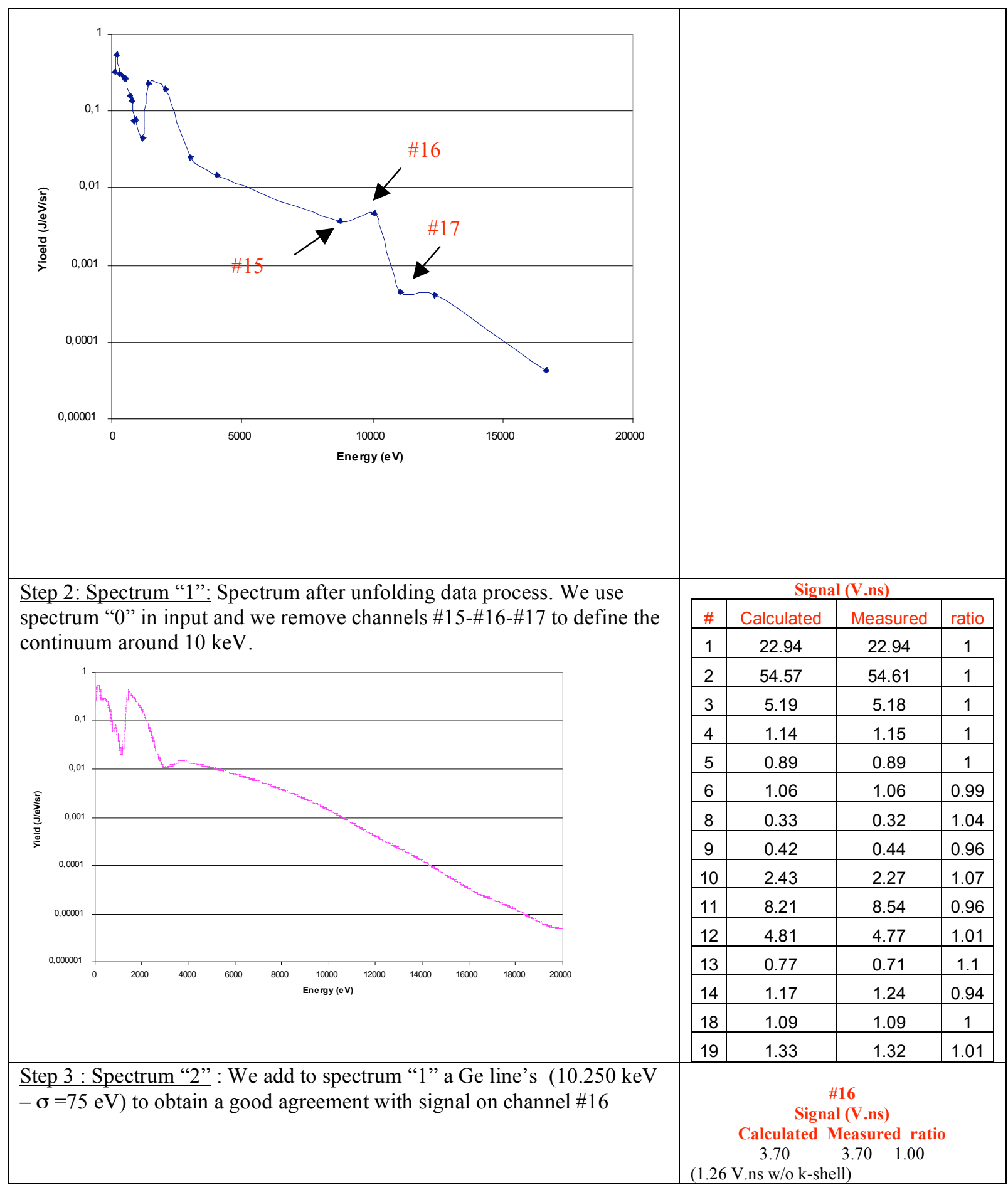




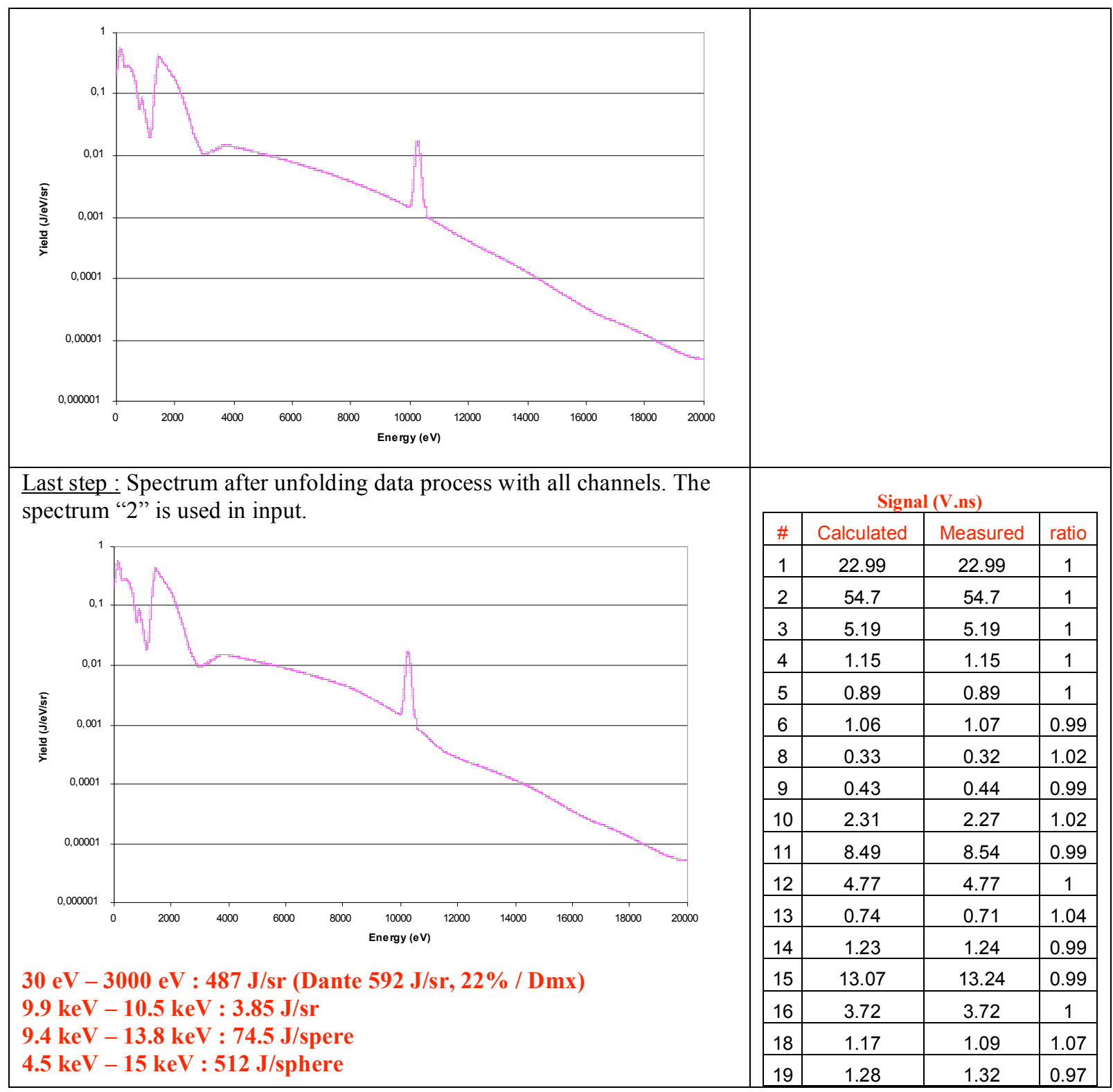

\section{$\underline{2}^{\text {nd }}$ method of analysis :}

(Always, without any assumption on the theoretical spectra)

It's the same as the first one, but now I take into account Ge k-shell an L-band emission. At the end of this treatment, I obtain a best agreement with Dande spectrum shape in 30 $\mathrm{eV}$ to $3 \mathrm{keV}$ range, that's I understand very well, but also on integrated yield !!! I don't understand why these 2 methods of analysis change so much the integrated yield between 30-3000 eV, from $487 \mathrm{~J} / \mathrm{sr}$ to $660 \mathrm{~J} / \mathrm{sr}$, whereas both spectra are in good agreement with the experimental data.

$\underline{\text { Step 1: Spectrum "0" }}$ 


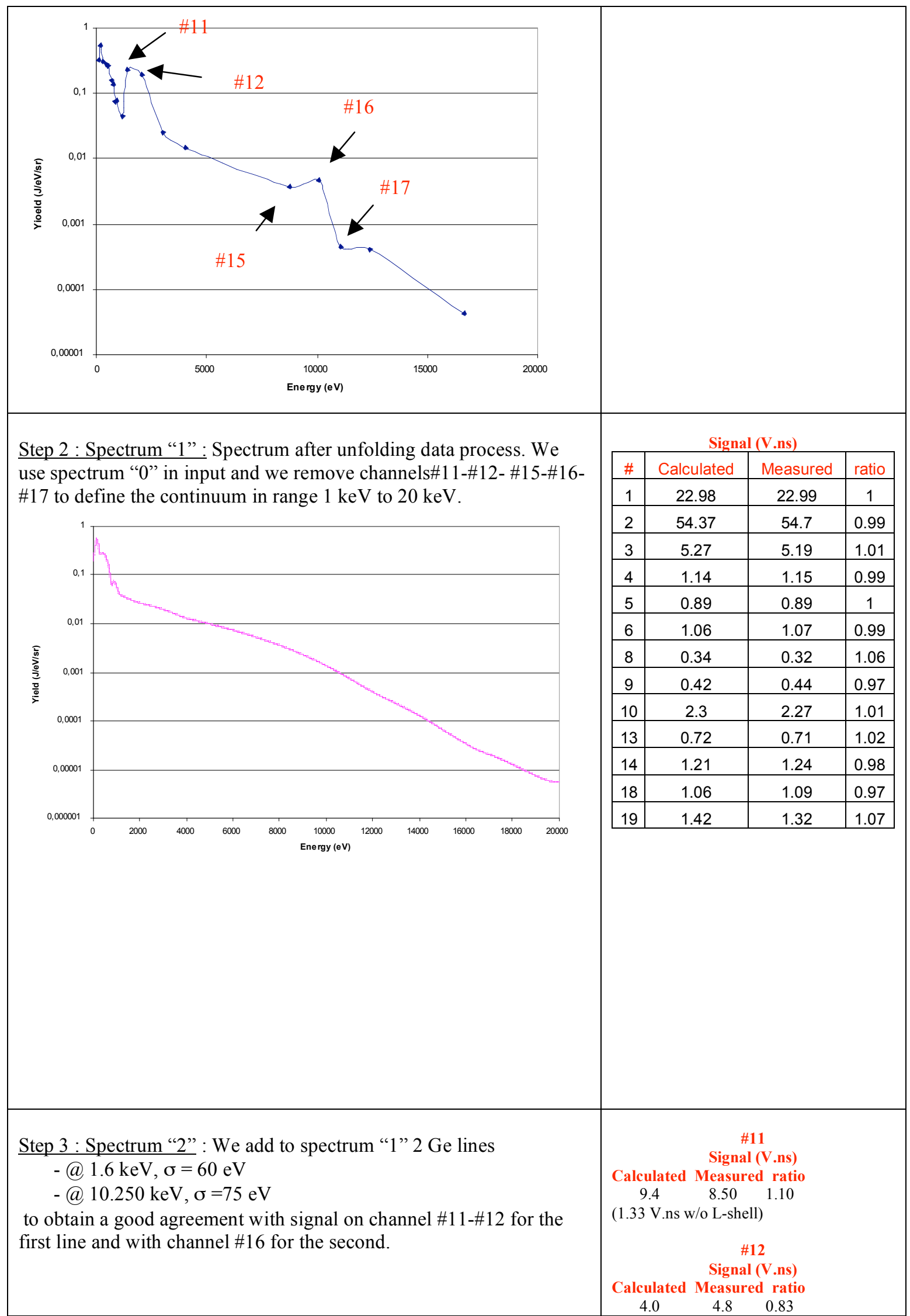




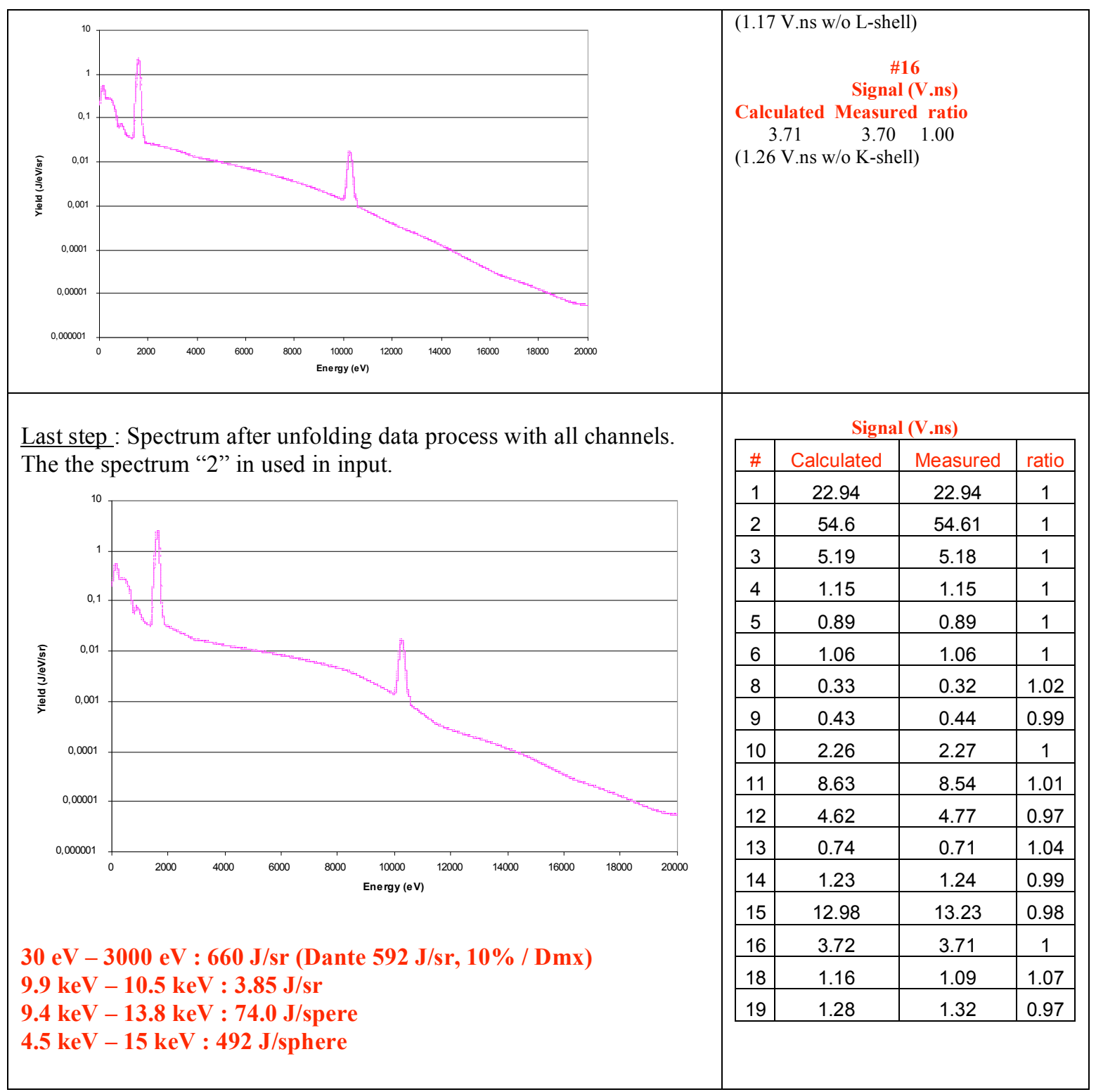

\section{$3^{\text {th }}$ method of analysis :}

This method consists to use a theoretical spectrum as the input of the iterative process. I think that you do that. In my case without theoretical spectrum, I've used the spectrum that I've obtained with the second method of analysis and I've added it a bump around $2.5 \mathrm{keV}$ to look like your spectrum shape. The result is shown on top. We find approximately the same thing as the second method of analysis, but we can observe a dip at $3 \mathrm{keV}$ which (I think) compensate the high intensity of the bump. 


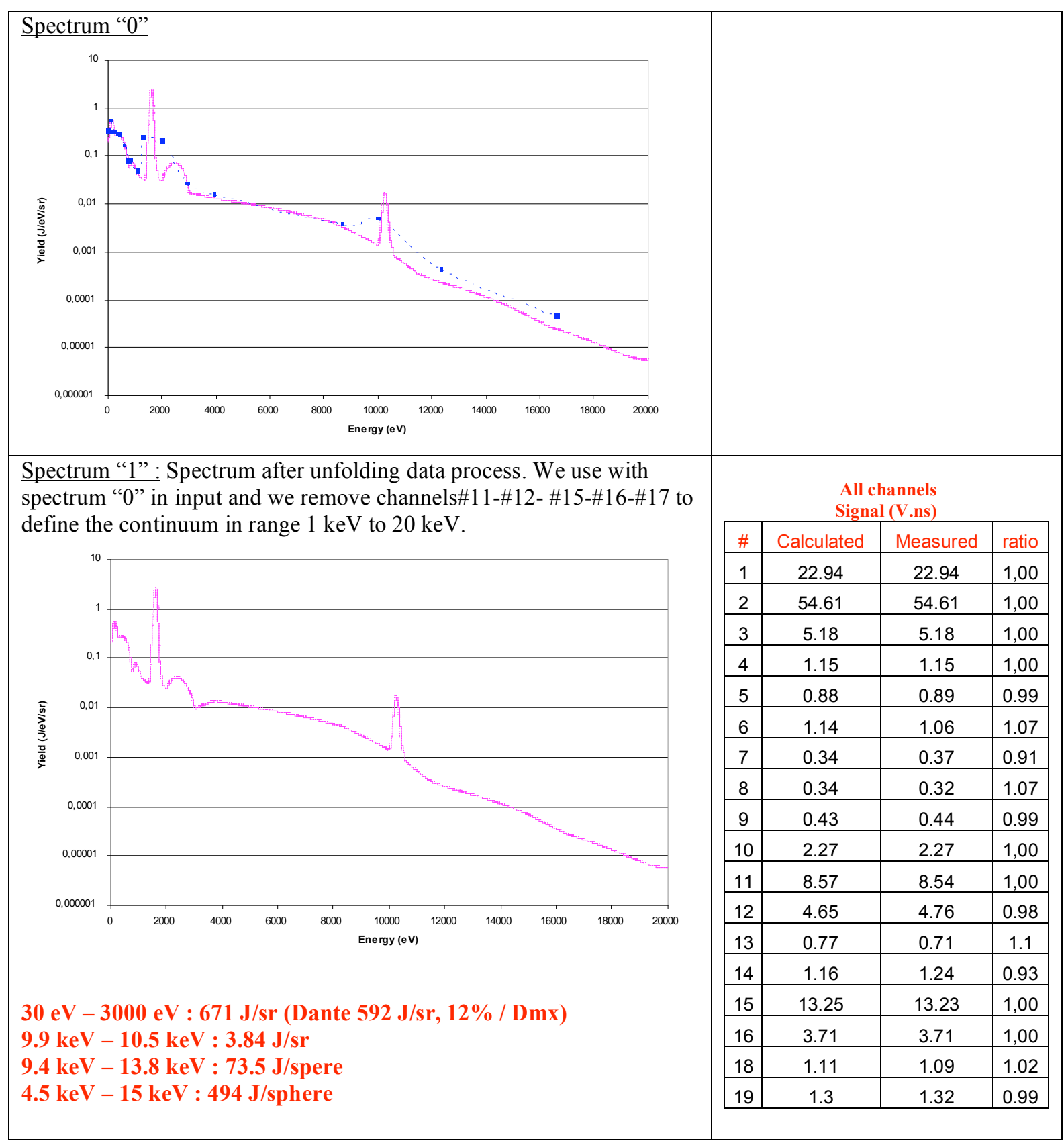

Summary: This graph show the results for shot 42751 that we obtain with the three methods 


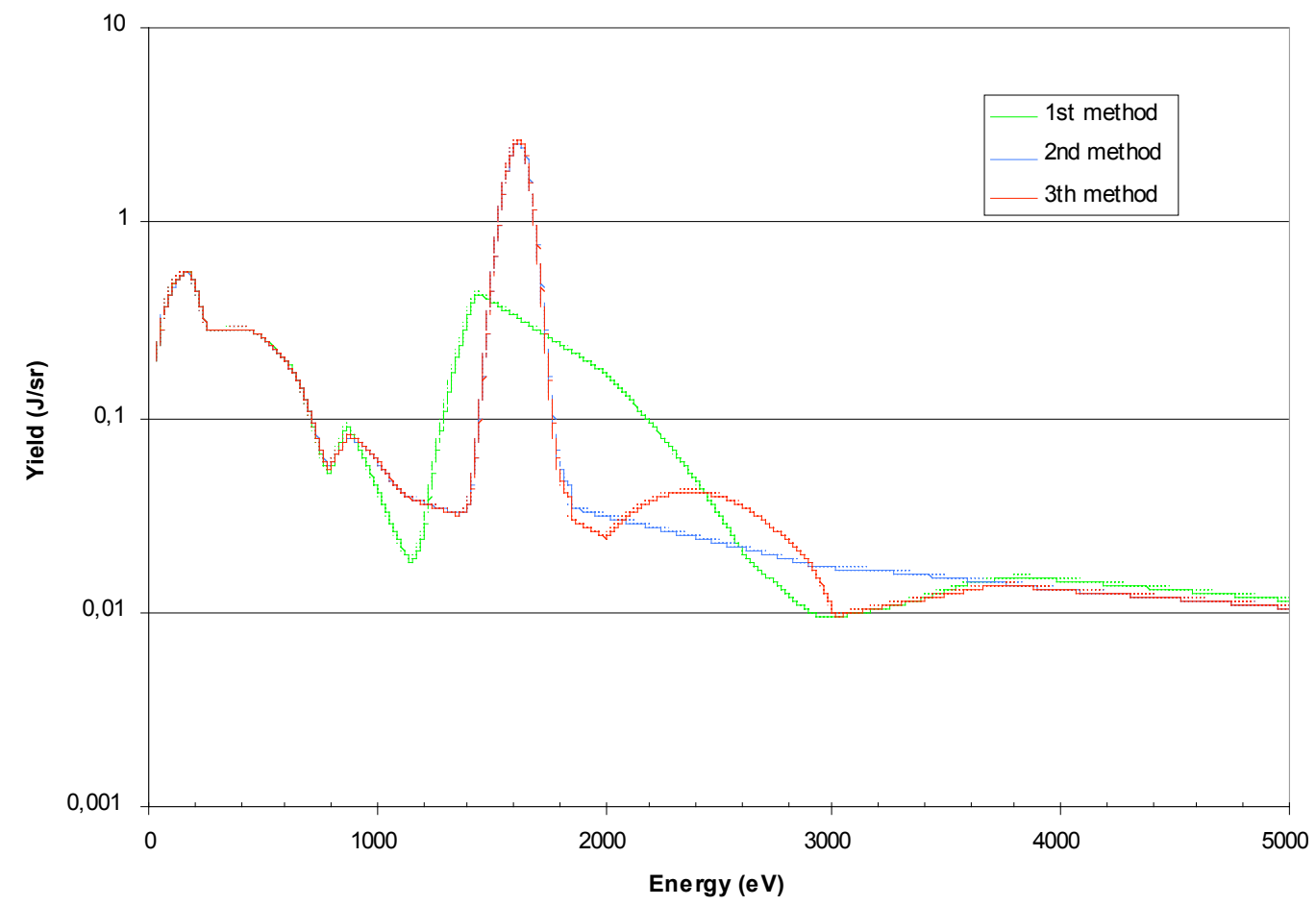

\begin{tabular}{|c|c|c|c|c|c|c|c|}
\hline Shot & Dante & \multicolumn{2}{|c|}{$\begin{array}{c}\text { Dmx } \\
\text { Method } 1\end{array}$} & \multicolumn{2}{|c|}{$\begin{array}{c}\text { Dmx } \\
\text { Method } 2\end{array}$} & \multicolumn{2}{|c|}{$\begin{array}{c}\text { Dmx } \\
\text { method } 3\end{array}$} \\
\hline \# & $(\mathrm{J} / \mathrm{sr})$ & $\mathrm{J} / \mathrm{sr}$ & \%difference & $\mathrm{J} / \mathrm{sr}$ & \%difference & $\mathrm{J} / \mathrm{sr}$ & \%difference \\
\hline 42749 & 756 & 525 & $6 \%$ & 712 & $6 \%$ & 734 & $3 \%$ \\
\hline 42750 & 797 & 536 & $13 \%$ & 706 & $13 \%$ & 720 & $11 \%$ \\
\hline 42751 & 592 & 486 & $10 \%$ & 662 & $10 \%$ & 671 & $12 \%$ \\
\hline 42752 & 868 & 595 & $6 \%$ & 815 & $6 \%$ & 821 & $6 \%$ \\
\hline 42753 & & & & & & & \\
\hline 42754 & 676 & 502 & $3 \%$ & 657 & $3 \%$ & 683 & $1 \%$ \\
\hline 42755 & 268 & 199 & $16 \%$ & 230 & $16 \%$ & 242 & $11 \%$ \\
\hline
\end{tabular}

Dante and Dmx yield from Ge-doped aerogel plasma radiation sources .Reported yields are integrated between $30 \mathrm{eV}$ and $3000 \mathrm{eV}$.

\begin{tabular}{|c|c|c|c|c|c|c|c|}
\hline Shot & Henway & \multicolumn{2}{|c|}{$\begin{array}{c}\text { Dmx } \\
\text { Method } 1\end{array}$} & \multicolumn{2}{|c|}{$\begin{array}{c}\text { Dmx } \\
\text { Method } 2\end{array}$} & \multicolumn{2}{|c|}{$\begin{array}{c}\text { Dmx } \\
\text { method } 3\end{array}$} \\
\hline \# & (J/sphere) & (J/sphere) & \%difference & (J/sphere) & \%difference & (J/sphere) & \%difference \\
\hline 42749 & $\sim 85$ & 82 & $4 \%$ & 82 & $4 \%$ & 80 & $6 \%$ \\
\hline 42750 & $\sim 170$ & 163 & $4 \%$ & 163 & $4 \%$ & 168 & $1 \%$ \\
\hline 42751 & $\sim 65$ & 74 & $12 \%$ & 74 & $12 \%$ & 73 & $11 \%$ \\
\hline 42752 & $\sim 57$ & 50 & $14 \%$ & 50 & $14 \%$ & 47 & $21 \%$ \\
\hline 42753 & & & & & & & \\
\hline 42754 & $\sim 110$ & 97 & $13 \%$ & 96 & $13 \%$ & 93 & $18 \%$ \\
\hline 42755 & $\sim 25$ & 52 & $52 \%$ & 52 & $52 \%$ & 50 & $50 \%$ \\
\hline
\end{tabular}

Henway and Dmx yield from Ge-doped aerogel plasma radiation sources .Reported yields are integrated between $9.4 \mathrm{keV}$ and $13.8 \mathrm{keV}$. 